\title{
Kimberley marine biota. Historical data: fishes
}

\author{
Glenn I. Moore ${ }^{1 *}$, Susan M. Morrison', J. Barry Hutchins', \\ Gerald R. Allen' and Alison Sampey' \\ ${ }^{1}$ Department of Aquatic Zoology, Western Australian Museum, Locked Bag 49, Welshpool DC, \\ Western Australia 6986, Australia \\ * Email: glenn.moore@museum.wa.gov.au
}

\begin{abstract}
Museum databases contain vast amounts of information that can help understand species distributions, patterns of biodiversity, taxonomic issues, evolutionary relationships and the effects of anthropogenic changes such as climate change. The marine waters of the Kimberley region in north-west Australia are among the least impacted marine ecosystems in the world, so there is a need to compile the extensive museum records for the region and make them more widely accessible. Here, we synthesise records of shallow water marine fish species in the Kimberley collected between 1880 and 2009. Based on 8,326 specimen-based records and thousands of reliable visual records from some 123 broad localities, a total of 1,475 species from 135 families were identified. Pronounced cross continental shelf differences in the fish communities exist with only $20 \%$ of species common to both inshore and offshore locales. Offshore atolls have very high species diversity, typified by wide ranging Indo-West Pacific species. In contrast, inshore reefs have lower diversity but support a much higher proportion of endemic species. The marine communities of the Kimberley face increasing pressure from resource development, fishing and tourism, so continued biodiversity and taxonomic research is essential to inform management decisions.
\end{abstract}

KEYWORDS: baseline data, biodiversity, natural history collections, north-west Australia, species inventory

\section{INTRODUCTION}

Records of which species exist at particular locations are fundamental to broader questions of biogeography, ecology and management (Pyke and Ehrlich 2010). Such records serve to map species distributions, understand patterns of biodiversity including hotspots and even to interpret evolutionary pathways (e.g. Hutchins 2001; Roberts et al. 2002; Mora et al. 2003; Briggs and Bowen 2013; Gaither and Rocha 2013). Importantly, these records can also provide historical baseline data to monitor the effects of anthropogenic changes such as climate change (Perry et al. 2005).

The marine waters of the Kimberley and offshore regions in north-west Australia are recognised as including some of the least impacted marine ecosystems in the world (Halpern et al. 2008). Despite this recognition, many of the important baseline biodiversity data for the region are stored in museum databases and/or presented in unpublished 'grey' literature. There is a need to compile these data and make them more widely accessible in order to inform management decisions in the Kimberley, which faces increasing pressure from resource development, fishing and tourism (Wood and Mills 2008; Pyke and Ehrlich 2010).

Some of the earliest accounts of fish collections from the Kimberley were made by Gray (1827), Richardson (1848) and Saville-Kent (1889). Several other smaller collections were made in the area in the late 19th and early 20th centuries, including during the Mjöberg expeditions (Rendahl 1921). It was not until the 1980s that comprehensive surveys by various Australian museums began to capture the true biodiversity of the region.

The Western Australian Museum (WAM) and other Australian natural science institutions have undertaken marine biodiversity surveys of a range of taxa in the Kimberley inshore and offshore waters (e.g. Berry 1986, 1993; Wells et al. 1995; Walker et al. 1996; Bryce et al. 1997; Walker 1997). Fishes have featured prominently in these surveys (Allen and Russell 1986; Allen 1992, 1993; Hutchins 1995, 1996, 1997, 1998; Morrison and Hutchins 1997; Moore and Morrison 2009), yet there is still much 
to uncover. New species of fishes continue to be described from the region (e.g. Johnson 2012) and several species complexes are thought to contain multiple taxa (personal observations). Recent surveys continue to add new records to the known fauna and to extend the range distributions of some species (unpublished data).

\section{AIM}

The aim of this project was to synthesise records of shallow water $(<30 \mathrm{~m})$ fish species in the Kimberley Project Area collected between 1880 and 2009, and to assess diversity trends and taxonomic and collection gaps in the region's fish fauna (see Sampey et al. 2014).

\section{METHODS}

The Kimberley Project Area encompasses an area west and north of the Kimberley coast (south of Broome to the Western Australia-Northern Territory border) extending beyond the $1000 \mathrm{~m}$ bathymetric contour, with the coastline forming a natural inshore boundary, as shown in Figure 1 (see Sampey et al. 2014 for a full explanation of the study area). This incorporates an offshore area greater than what is often considered 'Kimberley' (Wilson 2013).

Marine fish data for the Kimberley Project Area (intertidal to $30 \mathrm{~m}$ ) were sourced from the collection databases of WAM, Australian Museum (AMS) and Museum and Art Gallery of the Northern Territory (MAGNT), along with published species lists by reliable museum-based fish taxonomists in ten reports (Allen and Russell 1986; Allen 1992, 1993; Hutchins 1995, 1996, 1997, 1998; Hutchins et al. 1995; Morrison and Hutchins 1997; Moore and Morrison 2009). Specimens were not re-examined for this study. Records representing undescribed species were retained when it was clear that a taxonomist working on the group considered them as valid operational taxonomic units (OTU). Species records based on dubious identifications (e.g. from well outside their known distributions) were removed from further analyses. The numbers of species presented here differ marginally from those of Sampey et al. (2014) because our dataset was refined slightly after submission of that paper; however it has no effect on the general trends identified by those authors.

The species names and taxonomic affinities used in this treatment are based on the Australian Faunal Directory (ABRS 2014) and the Catalog of Fishes (Eschmeyer 2014). Full methodological details are provided in Sampey et al. (2014). Briefly, data from all sources mentioned above were collated into a single database and the provenance details verified. The locations of specimen records were mapped using ARCGIS v9 and ArcMap v9.3.

Throughout, 'inshore' refers to locations along the coast and the numerous islands and reefs found shoreward of the $50 \mathrm{~m}$ depth contour (Figure 1). 'Offshore' refers to the shelf edge atolls, which arise from deeper waters $(200-400 \mathrm{~m})$ along the continental margin (Figure 1).

Where possible, each species was coded for its known biogeographic range and habitat preferences based on Allen (2009), ABRS (2014) or Eschmeyer (2014). The categories used to code the distribution and preferred habitats of each species are listed in Table 1. A species may exploit several habitats, so multiple codes were used as appropriate.

\section{RESULTS}

A total of 8,326 registered specimen lots were included in the dataset (4,941 from WAM, 2,626 from MAGNT and 759 from AMS, which represents $59 \%, 32 \%$ and $9 \%$ of the total, respectively). Many thousands of additional visual records from across the region were included. The oldest specimen records used in this dataset dated from 1886 (a small collection from Derby by C. Lees, housed at AMS), and other early records included incidental collections by Saville-Kent in the 1880-1890s (also housed at AMS), and a mullet collected in 1900 (at WAM). The present data are derived from hundreds of collecting sites within 123 geographic localities (e.g. feature such as island or bay), of which eight are considered 'offshore' (Figure 1, Table 2).

A total of 1475 species were accepted into the dataset, of which 714 were recorded from inshore waters and 1061 from offshore waters (Appendix 1). This includes eight that were identified as an OTU. These species represent 135 families, the most speciose of which were: Gobiidae (185 species), Labridae (122), Pomacentridae (97), Apogonidae (81) and Serranidae (60). A further 42 species were recorded in the source databases, but were excluded from our dataset because we considered their identification to be dubious (Appendix 2). They are highlighted here because these potentially confusing records have been reported in other publications and/or databases.

The most diverse assemblage records were from the offshore sites: Ashmore Reef (809 species), Scott Reef (627 species) and Clerke Reef (477 species), however extensive assemblages were also identified from some inshore areas, such as Broome (281 species), Cassini Island (171 species) and Beagle Bay (144 species) (Table 2). Collection effort between 


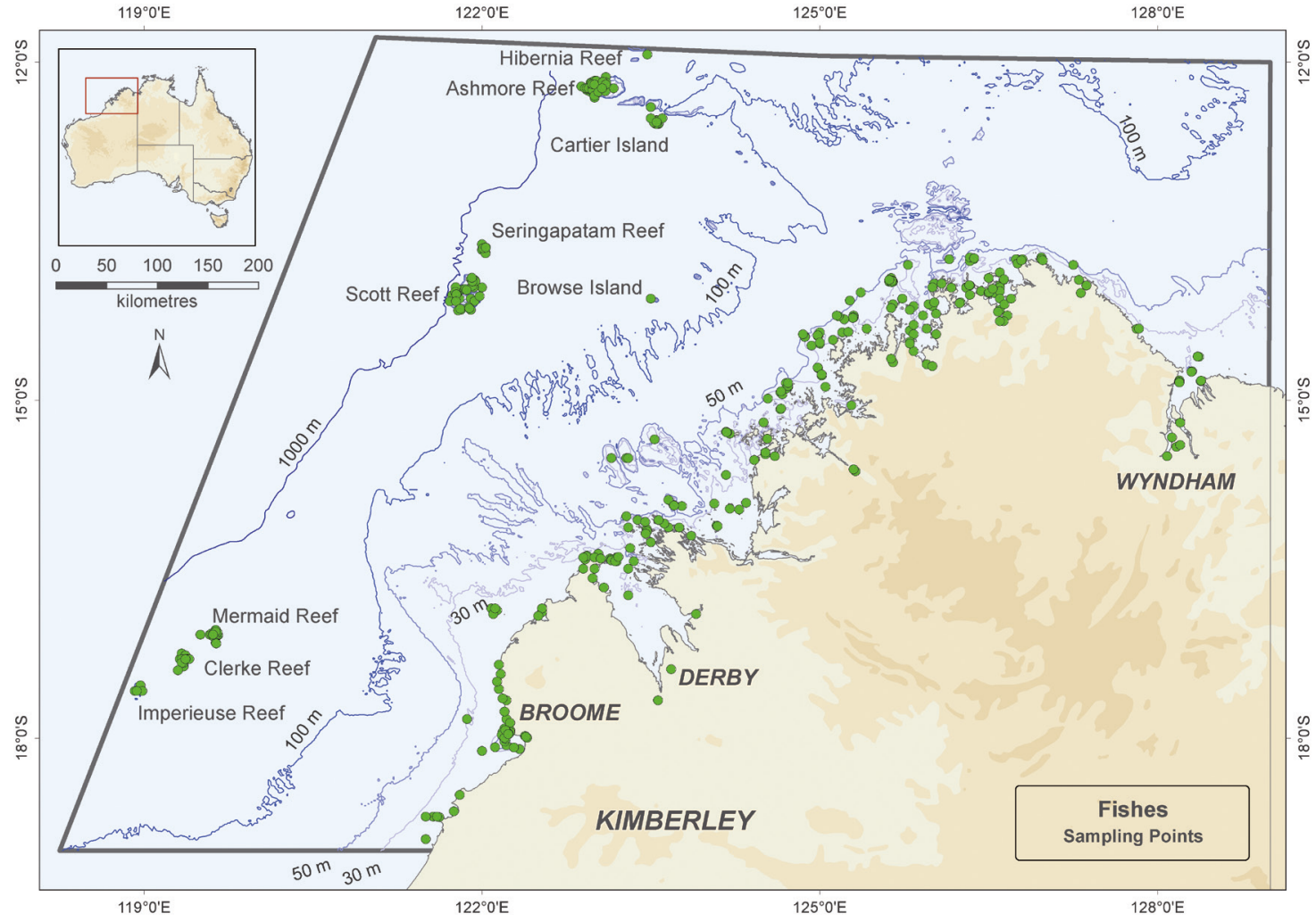

FIGURE 1 Location of historical records of fishes in the Kimberley Project Area of Western Australia. The Project Area boundary is marked in grey. Map projection: GDA94, Scale: 1:6, 250,000.

sites was not consistent (Table 2). Over half of the species (52\% or 761 species) recorded in the region are so far known only from offshore waters, while around $28 \%$ (414 species) are currently known only from inshore waters. The remaining 20\% (300 species) were recorded from both inshore and offshore regions.

Most species were considered to be widely distributed across the Indo-West Pacific (Table 3, Appendix 1). There were 98 inshore species (14\% of inshore species) considered endemic to Australia compared with only 24 offshore species ( $2 \%$ of offshore species) (Table 3). Eleven species are currently considered endemic to the Kimberley Project Area, namely Pseudanthias sheni, Assiculoides desmonotus, Pomacentrus limosus, Cirrhilabrus morrisoni, Conniella apterygia, Cirripectes alleni, Ecsenius alleni, Meiacanthus naevius, Stonogobiops larsonae, Xenisthmus chi and Xenisthmus semicinctus.

Based on the collated habitat preferences, more than $76 \%$ of all species in the dataset utilise hard substrates, $26 \%$ utilise soft substrates and around $14 \%$ include the water column (pelagic) among their habitat preferences (Table 4, Figure 3). Most offshore species prefer hard substrates, while both hard and soft substrates are broadly utilised by the inshore fish species (Table 4, Figure 3). More than $10 \%$ of the species may be found in estuarine or mangrove areas of the Kimberley, but these are generally inshore species (Table 4, Figure 3).

\section{DISCUSSION}

With nearly 1500 species, the Kimberley Project Area is home to around half of all species of fishes known from Western Australia. This diversity is broadly comparable to that of the Great Barrier Reef World Heritage Area (Hoese et al. 2006). Consequently it is a region of immense importance, with a need for ongoing biodiversity and biogeographic research. Here, we present an important first step in a comprehensive summary of major museum records to 2009. Other nonmuseum data exist for some shallow water parts of the Kimberley Project Area, particularly the offshore regions (e.g. Done et al. 1994; Kospartov et al. 2006) and the WAM Woodside Collection Project (Kimberley) 2008-2015 will provide additional records, collected in a semi quantitative manner, in the future (unpublished data). 
TABLE 1 Biogeographic and habitat codes assigned to fish species in the Kimberley Project Area dataset.

Code Definition

\section{BIOGEOGRAPHIC}

A Australian endemic. Found in tropical and temperate Australian waters.

C Circumglobal. Found in all oceans in either tropical or tropical/temperate waters.

IA Indo-Australian. Found in Australian and Indonesian waters, may extend to the Philippines and Japan.

IO Indian Ocean. Restricted to the Indian Ocean.

IP Indo-Pacific. Found in the Red Sea, Indian and Pacific Oceans.

IWP Indo-West Pacific. Found in the Red Sea, Indian and western Pacific Oceans. A few widespread species are also known from the Atlantic (IWP/AT).

NA Northern Australian endemic. Found in tropical Australian waters.

WA Western Australian endemic. Known only from Western Australian waters.

U Unknown. Used only for an undescribed OTU.

\section{HABITAT}
A Amphibious. Living along the land/water interface.
E Estuarine. Found in estuarine or brackish waters.
EnZ Endozoic. Always found in an internal association with a particular species of animal, i.e. living within another animal.
EZ Epizoic. Always found in an external association with a particular species of animal.
FW Freshwater. Found in freshwater. Only included in this dataset if also associated with another habitat.
$\mathrm{H} \quad$ Hard Substrate. Found associated with hard substrates (e.g. rock, coral, rubble).
M Mangrove. Found amongst mangroves.
$\mathrm{P} \quad$ Pelagic. Found in the water column.
S Soft Substrate. Found associated with soft substrates (e.g. sand, mud).
SG Seagrass. Found associated with seagrass meadows.

TABLE 2 Summary of locations of historical fish records, the range of years over which the records were collected, the number of collection events (see Sampey et al. 2014 for how this was determined), and the number of fish species and families known from each location.

\begin{tabular}{|c|c|c|c|c|}
\hline Location & Collecting Years & No. Coll. Events & No. Species & No. Families \\
\hline \multicolumn{5}{|l|}{ INSHORE SITES } \\
\hline Adele Island & 1952-1986 & 4 & 15 & 10 \\
\hline Admiral Island & 1994 & 1 & 30 & 17 \\
\hline Admiralty Gulf & 1968-1989 & 4 & 23 & 17 \\
\hline Albert Islands & 1996 & 1 & 1 & 1 \\
\hline Beagle Bay & 1997 & 1 & 144 & 53 \\
\hline Beagle Reef & 1991 & 1 & 88 & 32 \\
\hline Bedford Island & 1911-1994 & 3 & 42 & 23 \\
\hline Berthier Island & 1996 & 1 & 34 & 20 \\
\hline Bigge Island & 1996 & 1 & 24 & 12 \\
\hline Bonaparte Archipelago & 1978 & 1 & 16 & 13 \\
\hline Borda Island & 1968-1968 & 1 & 1 & 1 \\
\hline Brecknock Island & 1994 & 1 & 26 & 13 \\
\hline Broome & 1901-2004 & 56 & 281 & 90 \\
\hline Browse Island & 1949 & 1 & 1 & 1 \\
\hline Buffon Island & 1988 & 1 & 1 & 1 \\
\hline
\end{tabular}




\begin{tabular}{|c|c|c|c|c|}
\hline Location & Collecting Years & No. Coll. Events & No. Species & No. Families \\
\hline Caffarelli Island & 1994 & 1 & 20 & 9 \\
\hline Cambridge Gulf & 1891-1995 & 4 & 24 & 19 \\
\hline Camden Sound & 1990 & 1 & 1 & 1 \\
\hline Cape Boileau & 1982-1983 & 3 & 10 & 8 \\
\hline Cape Bossut & 1929-1982 & 4 & 12 & 10 \\
\hline Cape Domett & 1995 & 1 & 15 & 9 \\
\hline Cape Jaubert & 1929 & 1 & 2 & 2 \\
\hline Cape Leveque & 1929-1987 & 4 & 54 & 27 \\
\hline Cape Londonderry & 1946-1995 & 2 & 81 & 36 \\
\hline Cape Talbot & 1995 & 1 & 72 & 30 \\
\hline Careening Bay & 1994 & 1 & 25 & 18 \\
\hline Cassini Island & 1988-1998 & 5 & 171 & 38 \\
\hline Champagny Island & 1949 & 1 & 1 & 1 \\
\hline Churchill Reef & 1991-1996 & 2 & 75 & 28 \\
\hline Cockatoo Island & 1911-1963 & 4 & 9 & 8 \\
\hline Colbert Island & 1976-1996 & 2 & 30 & 16 \\
\hline Corneille Island & 1888 & 1 & 1 & 1 \\
\hline Coulomb Point & 1981-1983 & 2 & 34 & 23 \\
\hline Cygnet Bay & 1949-1996 & 2 & 28 & 17 \\
\hline De Freycinet Island & 1996 & 1 & 32 & 12 \\
\hline Derby & 1886-2003 & 13 & 28 & 19 \\
\hline Fenelon Island & 1985 & 1 & 8 & 7 \\
\hline Freshwater Bay & 1995 & 1 & 15 & 10 \\
\hline Gagg Island & 1994 & 1 & 28 & 13 \\
\hline Gibbings Reefs & 1996 & 1 & 39 & 18 \\
\hline Gourdon Bay & 1982-1983 & 2 & 8 & 7 \\
\hline Gregory Island & 1994 & 1 & 30 & 14 \\
\hline Hale Island & 1991 & 1 & 38 & 17 \\
\hline Hedley Island & 1996 & 1 & 24 & 14 \\
\hline Heritage Reef & 1991-1996 & 2 & 72 & 22 \\
\hline Ingram Shoals & 1998 & 1 & 8 & 4 \\
\hline Irvine Island & 1994 & 1 & 42 & 16 \\
\hline Jackson Island & 1994 & 1 & 1 & 1 \\
\hline James Price Point & 1981-1982 & 2 & 29 & 18 \\
\hline Jamieson Reef & 1996 & 1 & 44 & 13 \\
\hline Jar Island & 1995 & 1 & 43 & 24 \\
\hline Jones Island & 1991-1996 & 3 & 42 & 25 \\
\hline Jussieu Island & 1996 & 1 & 41 & 14 \\
\hline Kalumburu & 1900-1992 & 5 & 36 & 19 \\
\hline Kathleen Island & 1994 & 1 & 3 & 3 \\
\hline Keraudren Island & 1977 & 1 & 1 & 1 \\
\hline King Edward River & 1995 & 1 & 6 & 4 \\
\hline King George River & 1991-1995 & 2 & 19 & 16 \\
\hline King Sound & 1924-1986 & 3 & 10 & 8 \\
\hline Kingfisher Island & 1994-1996 & 2 & 53 & 31 \\
\hline Koolan Island & 1969-1981 & 2 & 2 & 2 \\
\hline Kuri Bay & 1964-1995 & 3 & 5 & 5 \\
\hline Lacepede Islands & 1945-1991 & 4 & 30 & 20 \\
\hline Lacrosse Island & 1995 & 1 & 14 & 11 \\
\hline Lamarck Island & 1996 & 1 & 39 & 22 \\
\hline Langey Crossing & 1966 & 1 & 1 & 1 \\
\hline Lawley River & 1976 & 1 & 8 & 6 \\
\hline Leonie Island & 1994 & 1 & 37 & 24 \\
\hline Lesueur Island & 1991 & 1 & 30 & 18 \\
\hline Long Island & 1995 & 1 & 48 & 21 \\
\hline
\end{tabular}




\begin{tabular}{|c|c|c|c|c|}
\hline Location & Collecting Years & No. Coll. Events & No. Species & No. Families \\
\hline Long Reef & 1991-1995 & 3 & 72 & 21 \\
\hline Lord Island & 1991-1994 & 2 & 34 & 18 \\
\hline Louis Islands & 1995 & 1 & 69 & 32 \\
\hline Lucas Island & 1988 & 1 & 1 & 1 \\
\hline Mackenzie Anchorage & 1995 & 1 & 25 & 14 \\
\hline Macleay Island & 1994-1996 & 3 & 36 & 17 \\
\hline Maret Islands & 1949-1996 & 2 & 34 & 15 \\
\hline Medusa Banks & 1968-1968 & 3 & 10 & 7 \\
\hline Mermaid Island & 1994 & 1 & 17 & 6 \\
\hline Mitchell River & 1968 & 1 & 1 & 1 \\
\hline Montalivet Islands & 1991-1996 & 2 & 70 & 20 \\
\hline Montesquieu Island & 2003 & 1 & 1 & 1 \\
\hline Montgomery Reef & 1994-1996 & 2 & 117 & 48 \\
\hline Myrmidon Ledge & 1995 & 1 & 17 & 13 \\
\hline Napier Broome Bay & 1968-1995 & 7 & 82 & 42 \\
\hline Osborne Islands & 1967 & 1 & 2 & 1 \\
\hline Parry Harbour & 1976-1991 & 2 & 2 & 2 \\
\hline Pender Bay & 1991 & 1 & 3 & 3 \\
\hline Port Warrender & 1976-1986 & 3 & 14 & 10 \\
\hline Powerful Island & 1991 & 1 & 62 & 32 \\
\hline Prince Frederick Harbour & 1973 & 1 & 1 & 1 \\
\hline Prince Regent River & 1974-1991 & 2 & 12 & 10 \\
\hline Prudhoe Islands & 1988-1996 & 2 & 34 & 14 \\
\hline Quondong Point & 1982 & 1 & 23 & 17 \\
\hline Reveley Island & 1995 & 1 & 26 & 20 \\
\hline Robroy Reefs & 1991-1996 & 2 & 96 & 26 \\
\hline Scorpion Island & 1991 & 1 & 38 & 20 \\
\hline Slate Islands & 1996 & 1 & 29 & 17 \\
\hline Solem Islands & 1991 & 1 & 73 & 24 \\
\hline Stokes Bay & 1917 & 1 & 1 & 1 \\
\hline Sunday Island & 1917-1994 & 4 & 123 & 40 \\
\hline Talbot Bay & 1994 & 1 & 17 & 10 \\
\hline Tallon Island & 1994 & 1 & 38 & 21 \\
\hline Troughton Island & & 1 & 1 & 1 \\
\hline Vansittart Bay & 1968-1995 & 3 & 32 & 22 \\
\hline Walsh Point & 1976 & 1 & 31 & 19 \\
\hline West Governor Island & 1995 & 1 & 41 & 22 \\
\hline Whirlpool Pass & 1994 & 1 & 50 & 31 \\
\hline White Island & 1996 & 1 & 21 & 11 \\
\hline Wildcat Reefs & 1996 & 1 & 66 & 27 \\
\hline Woodward Island & 1991 & 1 & 44 & 16 \\
\hline Wyndham & 1957-1985 & 7 & 20 & 16 \\
\hline Yampi Sound & 1945-1968 & 14 & 21 & 13 \\
\hline Yankawingarri Island & 1991 & 1 & 78 & 26 \\
\hline York Sound & 1975 & 1 & 3 & 3 \\
\hline \multicolumn{5}{|l|}{ OFFSHORE SITES } \\
\hline Ashmore Reef & 1972-1997 & 13 & 809 & 90 \\
\hline Cartier Island & 1949-1998 & 10 & 428 & 68 \\
\hline Clerke Reef & 1973-1993 & 7 & 477 & 66 \\
\hline Hibernia Reef & 1992-1998 & 5 & 352 & 55 \\
\hline Imperieuse Reef & 1973-1990 & 4 & 39 & 15 \\
\hline Mermaid Reef & 1973-2006 & 4 & 406 & 56 \\
\hline Scott Reef & 1908-2006 & 10 & 627 & 64 \\
\hline Seringapatam Reef & 1984-2006 & 4 & 296 & 43 \\
\hline
\end{tabular}


a. Inshore

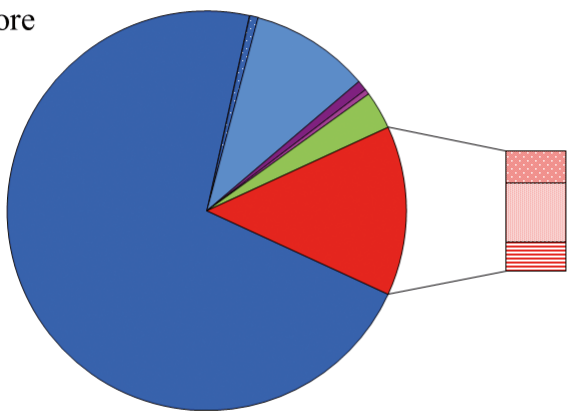

b. Offshore

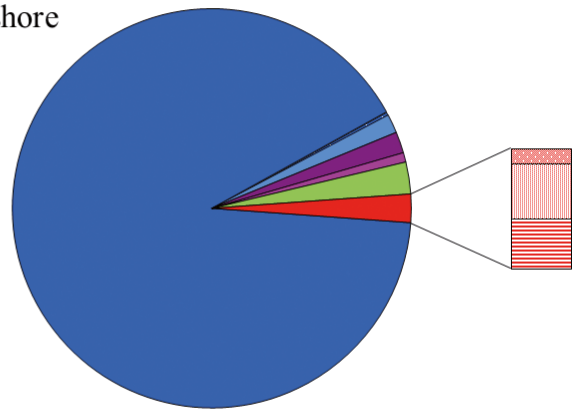

$\square$ IWP $\quad$ IWP/AT $\square$ IA $\square$ IP $\square$ IO $\square$ C $\square$ A $\square$ NA 自WA

FIGURE 2 Biogeographic affinities of all species of fishes in the Kimberley Project Area dataset. a. species recorded inshore; b. species recorded offshore. Australian endemics are pooled in the pie graph (represented by solid red) and expanded inset. Abbreviations are as follows: IWP, Indo-West Pacific; AT, Atlantic; IA, IndoAustralian; IO, Indian Ocean; C, circumglobal; A, Australia; NA, Northern Australia; WA, Western Australia.

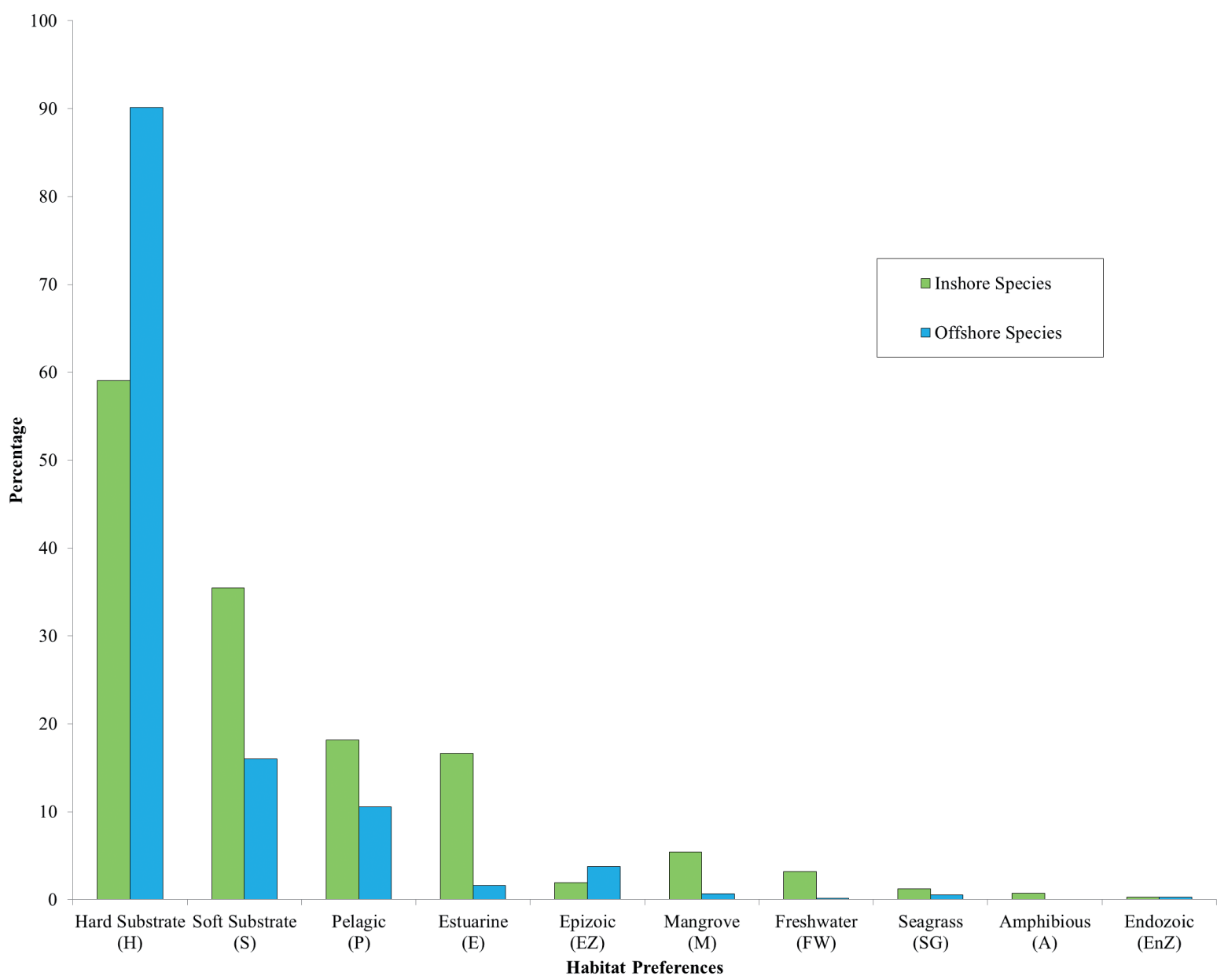

FIGURE 3 Habitat preferences of species of fishes recorded inshore and offshore in the Kimberley Project Area dataset. Note that many species utilise more than one habitat. Data presented in Table 4. 
TABLE 3 The biogeographical distribution of fish species recorded in the Kimberley Project Area dataset. Species with distributions marked by an asterisk $\left({ }^{*}\right)$ are considered endemic to Australia.

\begin{tabular}{lll} 
Biogeographic Region (Code) & Inshore Sites & Offshore Sites \\
\hline Australian (A) & 25 & 3 \\
Circumtropical (C) & 22 & 29 \\
Indo-Australian (IA) & 69 & 16 \\
Indian Ocean (IO) & 3 & 8 \\
Indo-Pacific (IP) & 6 & 18 \\
Indo-West Pacific (IWP) & 510 & 959 \\
Indo-West Pacific/Atlantic (IWP/AT) & 5 & 3 \\
Northern Australian (NA)* & 50 & 11 \\
Western Australian (WA)* & 23 & 10 \\
Unknown (U) & 1 & 4 \\
TOTAL & 714 & $\mathbf{1 0 6 1}$ \\
\hline
\end{tabular}

TABLE 4 The habitats of fish species recorded in the Kimberley Project Area dataset. Many species utilise several habitats and are therefore represented in multiple habitat counts (see Appendix 1). Data summarised in Figure 3.

\begin{tabular}{lllll} 
& \multicolumn{2}{c}{ Inshore Species } & \multicolumn{2}{c}{ Offshore Species } \\
Habitat & No. Species & $\%$ & No. Species & $\%$ \\
\hline Amphibious (A) & 5 & 0.7 & 0 & 0.0 \\
Estuarine (E) & 119 & 16.7 & 17 & 1.6 \\
Endozoic (EnZ) & 2 & 0.3 & 3 & 0.3 \\
Epizoic (EZ) & 14 & 2.0 & 40 & 3.8 \\
Freshwater (FW) & 23 & 3.2 & 2 & 0.2 \\
Hard Substrate (H) & 422 & 59.5 & 956 & 90.5 \\
Mangrove (M) & 39 & 5.5 & 7 & 0.7 \\
Pelagic (P) & 130 & 18.4 & 112 & 10.6 \\
Soft Substrate (S) & 252 & 35.7 & 170 & 16.1 \\
Seagrass (SG) & 9 & 1.3 & 6 & 0.6 \\
\hline
\end{tabular}

Most species in this dataset can be considered primarily reef associated. This is expected given that fish diversity and abundance is known to be strongly influenced by habitat complexity and benthic biological diversity (e.g. Gratwicke and Speight 2005; Komyakova et al. 2013). In the present dataset, this finding probably also partly reflects a bias towards sampling of hard substrate communities.

The marine fish fauna of the Kimberley Project Area is characterised by widespread species, most of which are found throughout the IndoWest Pacific. The prominent families are among the most speciose marine fish families in the region (Allen and Erdmann 2012). For other species (e.g. Chrysiptera hemicyanea, Halophryne ocellatus, Labracinus cyclophthalmus), the Kimberley is at the edge of their known distribution ranges.
Importantly, some 120 fish species recorded from the Kimberley Project Area are considered endemic to Australia, and 11 of these are restricted to the Kimberley. Endemism is particularly prominent among the inshore fauna. This level of endemism is relatively high for tropical Australian fish communities (Hoese et al. 2006). The offshore areas have had formal protection for some time and protection of the inshore reefs, which support the highest endemism, is in progress (MPRA 2013). At least one species in this data set (Pristis zijsron) is listed as threatened (Australian Government 1999). However, other threatened species are known to inhabit the area but have not been recorded by museum surveys (e.g. Rhincodon typus), and others are reliant on the estuarine systems of the Kimberley and not captured in the present dataset (e.g. Glyphis spp., Pristis spp.). 
Hutchins (1999) recognised longitudinal patterns in the composition of the inshore fish fauna in the Kimberley and it is beyond the scope of the present paper to revisit that analysis. However, the present data highlight a pronounced difference in species composition between inshore and offshore regions, with only about $20 \%$ of species shared between inshore and offshore regions. More than half of all species in the dataset have only been recorded on the offshore atolls, which support some of the greatest fish diversity and abundance in Australia (Allen 1993; Done et al. 1994; Hutchins 1998; Moore and Morrison 2009). Such faunal differences are expected and recognised (e.g. Allen and Russell 1986; Wilson 2014) because the areas are classified as different bioregions with contrasting physical and environmental conditions (Commonwealth of Australia 2006). The inshore Canning, Kimberley and Bonaparte Gulf Bioregions are recognised for their fringing rocky reefs and turbid water in a macrotidal setting. In contrast, the Oceanic Shoals Bioregion comprises shelf edge atolls and platform reefs in clear oceanic water. The differences in habitat preferences largely reflect this contrast.

Museum data contain a wealth of important information in the fields of taxonomy, biodiversity, biogeography, evolution and conservation. However there are well known limitations to using such data, due to the variable and largely qualitative manner in which it has often been collected. Considerable effort is being put into developing models and methods for utilising the information contained within them (e.g. Elith et al. 2006; Newbold 2010; Pyke and Ehrlich 2010). Given the nature of sampling and collecting, this dataset can largely be considered as 'presence only' data (see Graham et al. 2004). Although some of these data have been collected with a semi quantitative approach, the sampling effort has rarely been sufficient to confidently interpret the 'absence' of a species as meaningful (but see Done et al. 1994; Hutchins 2001; Moore and Morrison 2009 and others, for discussions of species abundances).

This synthesis of historical sampling highlights some collection gaps and biases. Firstly, as noted above, most of the recorded species are associated with hard substrates. However, vast expanses of the region are covered by soft sediment habitats (Wilson 2013), which have received comparatively little collection effort (but see Travers et al. 2012). Secondly, this dataset contains only a single shallow water record from the midshelf region (from Browse Island). The midshelf reefs and shoals are potentially informative because they are geographical midpoints between the inshore and offshore regions. Several of these locations have been visited recently by WAM, however much more sampling is required. Thirdly, there are inevitable taxonomic biases, with typically mobile, reef associated species dominating the data and nocturnal, cryptic or difficult to collect species probably under represented. In addition, this synthesis was part of a multi-taxon project (see Sampey et al. 2014), and as such was limited to shallow water specimens. Substantial collections of fishes from below $30 \mathrm{~m}$ exist in various institutions (e.g. WAM, CSIRO), although they are sometimes limited in terms of sampling effort and spatial scale.

Taxonomic decisions change frequently as new research is completed. Specimens were not reexamined for this study, and the vastness of the collections held by the contributing institutions means that some taxonomic errors are possible. For example, specimens examined prior to the resolution of species complexes might retain the original identification. In most instances, this was detected and updated as part of the present study. However, there were 1,783 instances of incomplete or confusing identifications that were excluded from the dataset. As noted above, 42 records were also excluded based on doubtful identifications. Any specimens associated with these records require re-examination.

This synthesis is a first step summary of the Kimberley marine fish fauna. We have identified several areas for future work, including:

- Continued identification of the nearly 2000 excluded records, and continued research into the taxonomy of unresolved species groups.

- Continued surveys of other parts of the Kimberley, including the mid-shelf shoals. To improve the utility of the data such surveys should be quantitative and include other environmental variables (e.g. the WAM Woodside Collection Project (Kimberley) 2008-2015).

- Increased attention on under represented species (i.e. small, cryptic, nocturnal) and excluded species (i.e. deeper than $30 \mathrm{~m}$ ), for it is these groups where future species discovery is likely to be most prolific (Mora et al. 2008; Eschmeyer et al. 2010).

- Application of some of the developing mathematical models and methods for utilising the biogeographic information contained within museum data (e.g. Elith et al. 2006; Phillips et al. 2009; Newbold 2010).

\section{ACKNOWLEDGEMENTS}

We are grateful to our colleagues Barry Russell, Helen Larson and Doug Hoese whose data contributed substantially to this study. We thank Mark McGrouther, Amanda Hay and Doug Hoese (AMS), Gavin Dally and Barry Russell (MAGNT) 
for resolving questionable species records in their institution's databases. We also thank Stacey Osborne and Albert Miles (WAM) for inspecting taxonomic and spatial information and data formatting. We thank Woodside Energy Ltd for their generous and ongoing support of WAM research.

\section{REFERENCES}

ABRS (2014). Australian Faunal Directory. Australian Biological Resources Study, Canberra. http://www. environment.gov.au/biodiversity/abrs/onlineresources/fauna/afd/index.html.

Allen, G.R. (1992). Part VIII. Fishes (pp. 62-74). In: Morgan, G.J. (ed.), Aquatic fauna of the Kimberley islands and reefs, Western Australia. Unpublished Report. Western Australian Museum: Perth.

Allen, G.R. (1993). Fishes of Ashmore Reef and Cartier Island. Records of the Western Australian Museum Supplement 44: 67-91.

Allen, G.R. (2009). Field guide to marine fishes of tropical Australia and South-east Asia. Western Australian Museum: Perth.

Allen, G.R. and Erdmann, M.V. (2012). Reef fishes of the East Indies. Tropical Reef Research: Perth.

Allen, G.R. and Russell, B.C. (1986). Faunal survey of the Rowley Shoals, Scott Reef and Seringapatam Reef north-western Australia. Part VII. Fishes. Records of the Western Australian Museum Supplement 25: 75-103.

Australian Government (1999). Environment protection and biodiversity conservation Act 1999. No. 91, as amended up to Act No. 31, 2014. Department of Environment: Canberra. Compiled 24 June 2014.

Berry, P.F. (ed.) (1986). Faunal survey of the Rowley Shoals, Scott Reef and Seringapatam Reef northwestern Australia. Records of the Western Australian Museum Supplement 25.

Berry, P.F. (ed.) (1993). Marine faunal surveys of Ashmore Reef and Cartier Island north-western Australia. Records of the Western Australian Museum Supplement 44.

Briggs, J.C. and Bowen, B.W. (2013). Marine shelf habitat: biogeography and evolution. Journal of Biogeography 40: 1023-1035.

Bryce, C.W., Hutchins, J.B. and Fromont, J. (1997). Restricted marine biological survey of the 'garden bottom' of Beagle Bay, Kimberley, Western Australia. Unpublished Report. Western Australian Museum: Perth.

Collette, B.B., McDowell, J.R. and Graves, J.E. (2006). Phylogeny of recent billfishes (Xiphioidei). Bulletin of Marine Science 79: 455-468.

Commonwealth of Australia (2006). A Guide to the integrated marine and coastal regionalisation of Australia version 4.0. Department of the Environment and Heritage: Canberra, Australia.

Done, T.J., Williams, D.M., Speare, P., Turak, E., Davidson, J., DeVantier, L.M., Newman, S.J. and Hutchins, J.B. (1994). Surveys of coral and fish communities at Scott Reef and Rowley Shoals. Australian Institute of Marine Science: Townsville.

Elith, J., Graham, C.H., Anderson, R.P., Dudík, M., Ferrier, S., Guisan, A., Hijmans, R.J., Huettmann, F.,
Leathwick, J.R., Lehmann, A., Li, J., Lohmann, L.G., Loizelle, B.A., Manion, G., Moritz, C., Nakamura, M., Nakazawa, Y., Overton, J.M., Peterson, A.T., Phillips, S.J., Richardson, K., Scachetti-Pereira, R., Schapire, R.E., Soberón, J., Williams, S., Wisz, M.S. and Zimmermann, N.E. (2006). Novel methods improve prediction of species' distributions from occurrence data. Ecography 29: 129-151.

Eschmeyer, W.N. (2014). Catalog of Fishes electronic version. http://research.calacademy.org/research/ ichthyology/catalog/fishcatmain.asp.

Eschmeyer, W.N., Fricke, R., Fong, J.D. and Polack, D.A. (2010). Marine fish diversity: history of knowledge and discovery (Pisces). Zootaxa 2525: 19-50.

Fraser, T.H. (2013). A new genus of cardinalfish (Apogonidae: Percomorpha), redescription of Archamia and resemblances and relationships with Kurtus (Kurtidae: Percomorpha). Zootaxa 3714: 1-63.

Fraser, T.H. and Allen, G.R. (2010). Cardinalfish of the genus Apogonichthyoides Smith, 1949 (Apogonidae) with a description of a new species from the WestPacific region. Zootaxa 2348: 40-56.

Fraser, T.H. and Randall, J.E. (2011). Two new species of Foa (Apogonidae) from the Pacific Plate, with redescriptions of Foa brachygramma and Foa fo. Zootaxa 2988: 1-27.

Gaither, M.R. and Rocha, L.A. (2013). Origins of species richness in the Indo-Malay-Philippine biodiversity hotspot: evidence for the centre of overlap hypothesis. Journal of Biogeography 40: 1638-1648.

Gon, O. and Allen, G.R. (2012). Revision of the IndoPacific cardinalfish genus Siphamia (Perciformes: Apogonidae). Zootaxa 3294: 1-84.

Graham, C.H., Ferrier, S., Huettman, F., Moritz, C. and Peterson, A.T. (2004). New developments in museumbased informatics and applications in biodiversity analysis. Trends in Ecology and Evolution 19: 497-503.

Gratwicke, B. and Speight, M.R. (2005). The relationship between fish species richness, abundance and habitat complexity in a range of shallow tropical marine habitats. Journal of Fish Biology 66: 650-667. DOI: 10.1111/j.0022-1112.2005.00629.x.

Gray, J.E. (1827). Pisces (pp. 435-437). In: King, P.P. (ed.), Narrative of a survey of the intertropical and western coasts of Australia performed between the years 1818 and 1822. Volume 2, appendix.

Halpern, B.S., Walbridge, S., Selkoe, K.A., Kappel, C.V., Micheli, F., D'Agrosa, C., Bruno, J.F., Casey, K.S., Ebert, C., Fox, H.E., Fujita, R., Heinemann, D., Lenihan, H.S., Madin, E.M.P., Perry, M.T., Selig, E.R., Spalding, M., Steneck, R. and Watson, R. (2008). A global map of human impact on marine ecosystems. Science 319: 948-952. DOI: 10.1126/science.1149345.

Hoese, D.F., Bray, D.J., Paxton, J.R. and Allen, G.R. (eds) (2006). Fishes. Zoological Catalogue of Australia. Number 35 (3 Vols.). Australian Biological Resources Study \& CSIRO Publishing, Collingwood.

Hutchins, J.B. (1995). Part 11. Fishes (pp. 137-149). In: Wells, F.E., Hanley, J.R. and Walker, D.I. (eds), Survey of the marine biota of the southern Kimberley islands, Western Australia. Unpublished Report. Western Australian Museum: Perth. 
Hutchins, J.B. (1996). Part 9. Fishes (pp. 75-84). In: Walker, D.I., Wells, F.E. and Hanley, J.R. (eds), Survey of the marine biota of the eastern Kimberley, Western Australia. Unpublished Report. Western Australian Museum: Perth.

Hutchins, J.B. (1997). Fish. (pp. 11-17). In: Bryce, C., Hutchins, J.B. and Fromont, J. (eds), Restricted marine biological survey of the 'garden bottom' of Beagle Bay. Unpublished Report. Western Australian Museum: Perth.

Hutchins, J.B. (1998). Survey of the fishes of Ashmore Reef. Report prepared for Parks Australia North.

Hutchins, J.B. (1999). Biogeography of the nearshore marine fish fauna of the Kimberley, Western Australia. Proceedings of the 5th Indo-Pacific Fish Conference, Nouméa, Society of French Ichthyologists.

Hutchins, J.B. (2001). Biodiversity of shallow reef fish assemblages in Western Australia using a rapid censusing technique. Records of the Western Australian Museum 20: 247-270.

Hutchins, J.B., Williams, D.M., Newman, S.J., Cappo, M. and Speare, P. (1995). New records of fishes for the Rowley Shoals and Scott/Seringapatam Reefs, off north-western Australia. Records of the Western Australian Museum 17: 119-123.

Jaafar, Z. and Larson, H.K. (2008). A New Species of Mudskipper, Periophthalmus takita (Teleostei: Gobiidae: Oxudercinae), from Australia,with a Key to the Genus. Zoological Science 25: 946-952.

Johnson, J.W. (2012). Pseudopataecus carnatobarbatus, a new species of velvetfish (Teleostei: Scorpaeniformes: Aploactinidae) from the Kimberley coast of Western Australia. Zootaxa 3245: 54-62.

Komyakova, V., Munday, P.L. and Jones, G.P. (2013). Relative importance of coral cover, habitat complexity and diversity in determining the structure of reef fish communities. PloS One 8: e83178. DOI: 10.1371/ journal.pone.0083178.

Kospartov, M., Beger, M., Ceccarelli, D. and Richards, Z. (2006). An assessment of the distribution and abundance of sea cucumbers, trochus, giant clams, coral, fish and invasive marine species at Ashmore Reef National Nature Reserve and Cartier Island Marine Reserve: 2005. Unpublished report for The Department of the Environment and Heritage. UniQuest Pty Ltd: St. Lucia, Queensland.

Mabuchi, K., Fraser, T.H., Song, H., Azuma, Y. and Nishida, M. (2014). Revision of the systematics of the cardinalfishes (Percomorpha: Apogonidae) based on molecular analyses and comparative reevaluation of morphological characters. Zootaxa 3846: 151-203.

Moore, G.I. and Morrison, S.M. (2009). Fishes of three North West Shelf atolls off Western Australia: Mermaid (Rowley Shoals), Scott and Seringapatam Reefs. Records of the Western Australian Museum Supplement 77: 221-255.

Mora, C., Chittaro, P.M., Sale, P.F., Kritzer, J. and Ludsin, S.A. (2003). Patterns and processes in reef fish diversity. Nature 421: 933-936. DOI: 10.1038/ nature01393.

Mora, C., Tittensor, D.P. and Myers, R.A. (2008). The completeness of taxonomic inventories for describing the global diversity and distribution of marine fishes. Proceedings of the Royal Society B 275: 149-155.
Morrison, S.M. and Hutchins, J.B. (1997). Part 8. Fishes (pp. 67-76). In: Walker, D.I. (ed.), Marine biological survey of the central Kimberley coast, Western Australia. Unpublished Report. Western Australian Museum: Perth.

Motomura, H., Last, P.R. and Johnson, J.W. (2008). Review of the waspfish genus Liocranium (Scorpaeniformes: Tetrarogidae), with restoration of L. pleurostigma (Weber). Zootaxa 1820: 27-40.

MPRA (2013). Annual Report 2012-2013. Marine Parks and Reserves Authority: Kensington, Western Australia.

Newbold, T. (2010). Applications and limitations of museum data for conservation and ecology, with particular attention to species distribution models. Progress in Physical Geography 34: 3-22.

Perry, A.L., Low, P.J., Ellis, J.R. and Reynolds, J.D. (2005). Climate change and distribution shifts in marine fishes. Science 308: 1912-1915.

Phillips, S.J., Dudík, M., Elith, J., Graham, C.H., Lehmann, A., Leathwick, J. and Ferrier, S. (2009). Sample selection bias and presence-only distribution models: implications for background and pseudo-absence data. Ecological Applications 19: 181-197.

Pyke, G.H. and Ehrlich, P.R. (2010). Biological collections and ecological/environmental research: a review, some observations and a look to the future. Biological Reviews 85: 247-266.

Randall, J.E. (2007). Leptachirus, a new soleid fish genus from New Guinea and northern Australia, with descriptions of eight new species. Records of the Western Australian Museum 24: 81-108.

Rendahl, C.H. (1921). Results of Dr. E. Mjöberg's Swedish scientific expeditions to Australia, 1910-1913, Fische. Kungliga Svenska Vetenskaps-Akademiens Handlingar $\mathbf{6 1}$ : 1-24.

Richardson, J. (1848). Ichthyology. The Zoology of the voyage of H.M.S. 'Erebus' $\mathcal{E}$ 'Terror' under the command of Captain Sir James Clark Ross, R.N., F.R.S., during the years 1839-1843. Richardson, J. and Gray, J.E. (eds). E.W. Janson: London.

Roberts, M.R., McClean, C.J., Veron, J.E.N., Hawkins, J.P., Allen, G.R., McAllister, D.E., Mittermeier, C.G., Schueler, F.W., Spalding, M., Wells, F.E., Vynne, C. and Werner, T.B. (2002). Marine biodiversity hospots and conservation priorities for tropical reefs. Science 295: $1280-1284$.

Sampey, A., Bryce, C.W., Osborne, S. and Miles, A. (2014). Kimberley marine biota. Historical data: introduction and methods. Records of the Western Australian Museum Supplement 84: 19-43.

Saville-Kent, W. (1889). Preliminary observations on a natural history collection made in connection with the surveying cruise of H.M.S. 'Myrmidon', at Port Darwin and Cambridge Gulf. Proceedings of the Royal Society of Queensland 6: 219-242.

Travers, M.J., Potter, I.C., Clarke, K.R. and Newman, S.J. (2012). Relationships between latitude and environmental conditions and the species richness, abundance and composition of tropical fish assemblages over soft substrata. Marine Ecology Progress Series 446: 221-241. 
Walker, D.I. (1997). Marine biological survey of the central Kimberley coast, Western Australia. Unpublished Report. Western Australian Museum: Perth.

Walker, D.I., Wells, F.E. and Hanley, J.R. (1996). Survey of the marine biota of the eastern Kimberley, Western Australia. Unpublished Report. Western Australian Museum: Perth.

Wells, F.E., Hanley, J.R. and Walker, D.I. (1995). Survey of the marine biota of the southern Kimberley islands, Western Australia. Unpublished Report. Western Australian Museum: Perth.

White, W.T. (2012). A redescription of Carcharhinus dussumieri and C. sealei, with resurrection of $C$. coatesi and C. tjutjot as valid species (Chondrichthyes: Carcharhinidae). Zootaxa 3241: 1-34.

Wilson, B. (2013). The biogeography of the Australian North West Shelf: environmental change and life's response. Elsevier: Burlington, Massachusetts.

Wilson, B. (2014). Kimberley marine biota. History and environment. Records of the Western Australian Museum Supplement 84: 1-18.

Wood, M. and Mills, D. (2008). A turning of the tide: science for decisions in the Kimberley-Browse marine region. Western Australian Marine Science Institute: Perth.

MANUSCRIPT RECEIVED 25 JULY 2014; ACCEPTED 9 SEPTEMBER 2014. 
APPENDIX 1 Species of fishes recorded in the Kimberley Project Area dataset. Habitat and biogeographic codes are provided in the Methods. Superscripts denote a comment in footnotes.

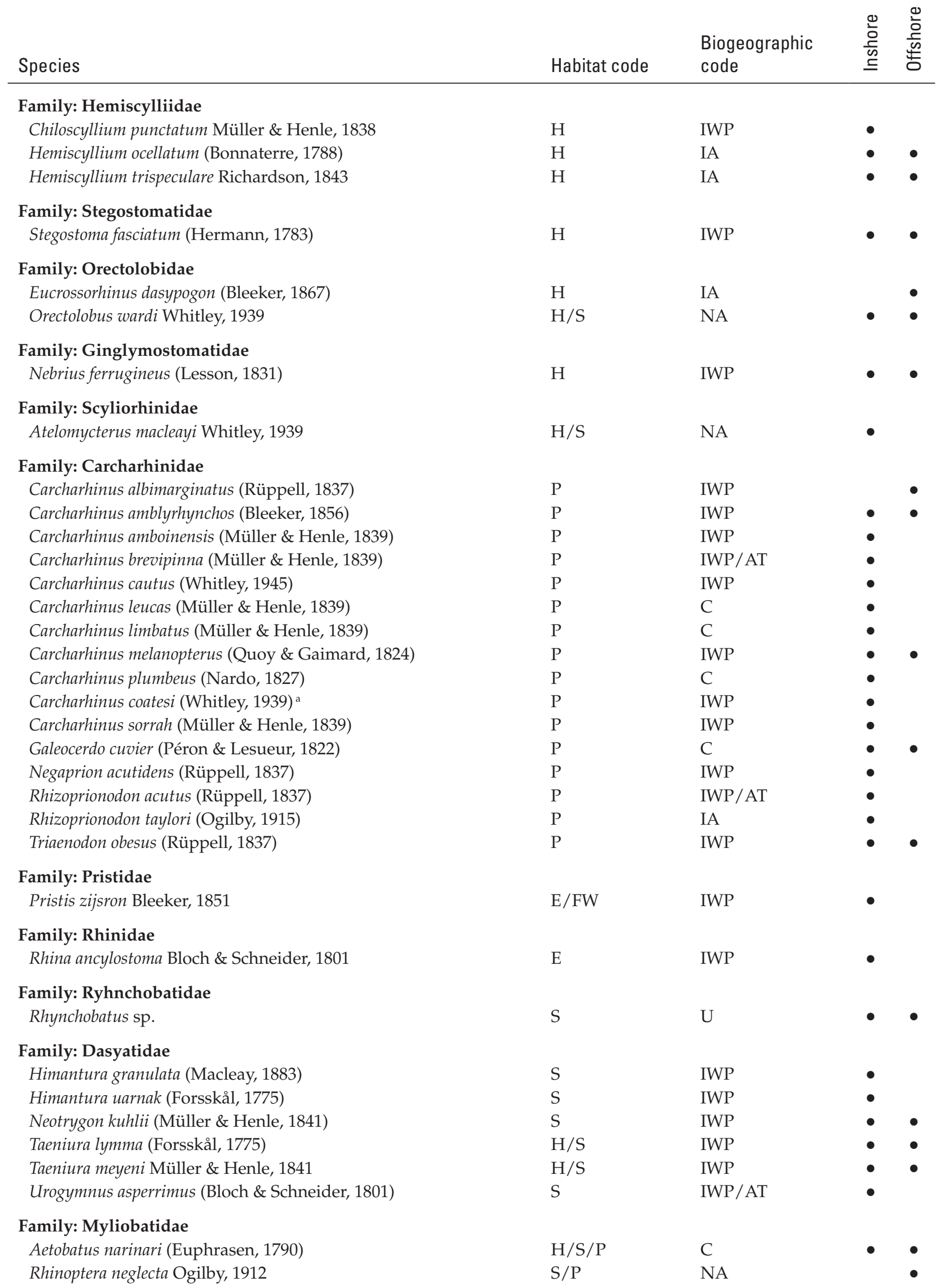




Species $\quad$ Habitat code $\quad \begin{array}{lll}0 \\ \text { code }\end{array}$

Family: Mobulidae

Manta birostris (Walbaum, 1792)

Family: Elopidae

Elops hawaiensis Regan, 1909

Family: Megalopidae

Megalops cyprinoides (Broussonet, 1782)

Family: Albulidae

Albula argentea (Forster, 1801) ${ }^{\mathrm{b}}$

Family: Anguillidae

Anguilla bicolor McClelland, 1844

Family: Moringuidae

Moringua abbreviata (Bleeker, 1863)

Moringua ferruginea Bliss, 1883

Moringua javanica (Kaup, 1856)

Moringua microchir Bleeker, 1853

Family: Chlopsidae

Kaupichthys atronasus Schultz, 1953

Kaupichthys brachychirus Schultz, 1953

Kaupichthys hyoproroides (Strömman, 1896)

\section{Family: Muraenidae}

Anarchias allardicei Jordan \& Starks, 1906

Anarchias seychellensis Smith, 1962

Echidna nebulosa (Ahl, 1789)

Echidna polyzona (Richardson, 1845)

Echidna unicolor Schultz, 1953

Enchelycore bayeri (Schultz, 1953)

Gymnomuraena zebra (Shaw \& Nodder, 1797)

Gymnothorax buroensis (Bleeker, 1857)

Gymnothorax chilospilus Bleeker, 1865

Gymnothorax enigmaticus McCosker \& Randall, 1982

Gymnothorax favagineus Bloch \& Schneider, 1801

Gymnothorax fimbriatus (Bennett, 1832)

Gymnothorax flavimarginatus (Rüppell, 1830)

Gymnothorax fuscomaculatus (Schultz, 1953)

Gymnothorax gracilicauda Jenkins, 1903

Gymnothorax javanicus (Bleeker, 1859)

Gymnothorax longinquus (Whitley, 1948)

Gymnothorax margaritophorus Bleeker, 1865

Gymnothorax melatremus Schultz, 1953

Gymnothorax monochrous (Bleeker, 1856)

Gymnothorax pictus (Ahl, 1789)

Gymnothorax pindae Smith, 1962

Gymnothorax pseudothyrsoideus (Bleeker, 1852)

Gymnothorax rueppellii (McClelland, 1844)

Gymnothorax thrysoideus (Richardson, 1845)

Gymnothorax undulatus (Lacépède, 1803)

Gymnothorax zonipectis Seale, 1906

Rhinomuraena quaesita Garman, 1888

Uropterygius concolor Rüppell, 1838

$P$

P

C

P IWP

$\mathrm{P} / \mathrm{E} / \mathrm{FW} \quad$ IWP

S/E/M IWP

H/S/E/FW IWP

$\mathrm{H} \quad$ IWP

$\mathrm{H} \quad$ IWP

$\mathrm{H} \quad$ IWP

$\mathrm{H} \quad$ IWP

$\mathrm{H} \quad$ IWP

$\mathrm{H} \quad$ IWP

$\mathrm{H} \quad$ IWP/AT

$\mathrm{H} \quad$ IWP

$\mathrm{H} \quad$ IWP

$\mathrm{H} \quad \mathrm{IP}$

$\mathrm{H} \quad$ IWP

$\mathrm{H} \quad$ IWP

$\mathrm{H} \quad$ IWP

$\mathrm{H}$ IP

$\mathrm{H}$ IP

$\mathrm{H} \quad$ IWP

$\mathrm{H} \quad$ IWP

$\mathrm{H} \quad$ IWP

$\mathrm{H} \quad$ IWP

$\mathrm{H} \quad \mathrm{IP}$

$\mathrm{H} \quad$ IWP

$\mathrm{H} \quad$ IWP

$\mathrm{H} \quad \mathrm{IP}$

$\mathrm{H} / \mathrm{S} \quad$ IWP

$\mathrm{H} \quad$ IWP

$\mathrm{H} \quad$ IWP

$\mathrm{H} \quad$ IWP

$\mathrm{H}$ IP

$\mathrm{H} \quad$ IWP

$\mathrm{H} \quad$ IWP

$\mathrm{H} \quad$ IWP

$\mathrm{H} \quad$ IWP

$\mathrm{H}$ IP

$\mathrm{H} \quad$ IWP

$\mathrm{H} / \mathrm{S} \quad$ IWP

$\mathrm{H}$ IWP 


\begin{tabular}{|c|c|c|c|}
\hline Species & Habitat code & $\begin{array}{l}\text { Biogeographic } \\
\text { code }\end{array}$ & 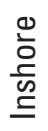 \\
\hline Uropterygius fuscoguttatus Schultz, 1953 & $\mathrm{H}$ & IWP & \\
\hline Uropterygius kamar McCosker \& Randall, 1977 & $\mathrm{H}$ & IWP & \\
\hline Uropterygius marmoratus (Lacépède, 1803) & $\mathrm{H}$ & IWP & \\
\hline Uropterygius xanthopterus Bleeker, 1859 & $\mathrm{H}$ & IWP & \\
\hline
\end{tabular}

Family: Muraenesocidae

Muraenesox bagio (Hamilton, 1822)

Muraenesox cinereus (Forsskål, 1775)

$\begin{array}{ll}\mathrm{E} & \text { IW } \\ \mathrm{H} / \mathrm{S} & \mathrm{IW} \\ & \\ \mathrm{S} & \mathrm{IW} \\ \mathrm{H} / \mathrm{S} & \mathrm{IW} \\ \mathrm{S} & \mathrm{U} \\ \mathrm{S} & \mathrm{IW}\end{array}$

\section{Family: Congridae}

Ariosoma scheelei (Strömman, 1896)

Conger cinereus Rüppell, 1830

Gnathophis sp.

Heteroconger hassi (Klausewitz \& Eibl-Eibesfeldt, 1959)

\section{Family: Ophichthidae}

Brachysomophis cirrocheilos (Bleeker, 1857)

Brachysomophis crocodilinus (Bennett, 1833)

Callechelys catostoma (Forster, 1801)

Callechelys marmorata (Bleeker, 1853)

Leiuranus semicinctus (Lay \& Bennett, 1839)

Muraenichthys thompsoni Jordan \& Richardson, 1908

Myrichthys maculosus (Cuvier, 1816)

Myrophis microchir (Bleeker, 1865)

Ophichthus altipennis (Kaup, 1856)

Ophichthus cephalozona (Bleeker, 1864)

Ophichthus lithinus (Jordan \& Richardson, 1908)

Ophichthus rutidoderma (Bleeker, 1853)

Phyllophichthus xenodontus Gosline, 1951

Pisodonophis boro (Hamilton, 1822)

Pisodonophis cancrivorus (Richardson, 1848)

Schismorhynchus labialis (Seale, 1917)

Scolecenchelys gymnota (Bleeker, 1857)

Scolecenchelys macroptera (Bleeker, 1857)

$\begin{array}{ll}\mathrm{H} / \mathrm{S} & \text { IWP } \\ \mathrm{H} / \mathrm{S} & \text { IWP } \\ \mathrm{H} / \mathrm{S} & \text { IWP } \\ \mathrm{H} / \mathrm{S} & \text { IWP } \\ \mathrm{H} / \mathrm{S} & \text { IWP } \\ \mathrm{S} / \mathrm{E} / \mathrm{M} & \text { IWP } \\ \mathrm{H} / \mathrm{S} & \mathrm{IWP} \\ \mathrm{S} / \mathrm{SG} & \text { IWP } \\ \mathrm{S} & \mathrm{IWP} \\ \mathrm{S} & \text { IWP } \\ \mathrm{S} & \text { IWP } \\ \mathrm{H} / \mathrm{S} & \text { IWP } \\ \mathrm{H} / \mathrm{S} & \text { IWP } \\ \mathrm{S} & \text { IWP } \\ \mathrm{S} / \mathrm{E} & \text { IWP } \\ \mathrm{S} & \text { IWP } \\ \mathrm{H} / \mathrm{S} & \text { IWP } \\ \mathrm{H} / \mathrm{S} & \text { IWP }\end{array}$

\section{Family: Clupeidae}

Amblygaster leiogaster (Valenciennes, 1847)

Anodontostoma chacunda (Hamilton, 1822)

Dussumieria elopsoides Bleeker, 1849

Herklotsichthys blackburni (Whitley, 1948)

Herklotsichthys koningsbergeri (Weber \& de Beaufort, 1912)

Nematalosa come (Richardson, 1846)

Nematalosa vlaminghi (Munro, 1957)

Pellona ditchela Valenciennes, 1847

Sardinella albella (Valenciennes, 1847)

Sardinella gibbosa (Bleeker, 1849)

Spratelloides delicatulus (Bennett, 1832)

Spratelloides gracilis (Schlegel, 1846)

Spratelloides robustus Ogilby, 1897

IWP

$P \quad$ IWP

P IWP

P WA

$\mathrm{P} \quad \mathrm{IA}$

P IWP

P WA

$\mathrm{P} \quad$ IWP

$\mathrm{P} \quad$ IWP

$\mathrm{P} \quad$ IWP

$\mathrm{P} \quad$ IWP

$\mathrm{P} \quad$ IWP

$\mathrm{P}$

A

Family: Pristigasteridae

Ilisha striatula Wongratana, 1983

Family: Engraulidae 


\begin{tabular}{|c|c|c|c|}
\hline Species & Habitat code & $\begin{array}{l}\text { Biogeographic } \\
\text { code }\end{array}$ & 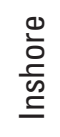 \\
\hline Stolephorus indicus (Hasselt, 1823) & $\mathrm{P}$ & IWP & $\bullet$ \\
\hline Thryssa aestuaria (Ogilby, 1910) & $\mathrm{P}$ & IA & $\bullet$ \\
\hline Thryssa hamiltonii (Gray, 1835) & $\mathrm{P}$ & IWP & $\bullet$ \\
\hline Thryssa scratchleyi (Ramsay \& Ogilby, 1886) & $\mathrm{P}$ & IA & $\bullet$ \\
\hline \multicolumn{4}{|l|}{ Family: Chirocentridae } \\
\hline Chirocentrus dorab (Forsskål, 1775) & $\mathrm{P}$ & IWP & $\bullet$ \\
\hline \multicolumn{4}{|l|}{ Family: Synodontidae } \\
\hline Harpadon translucens Saville-Kent, 1889 & $\mathrm{H} / \mathrm{S}$ & IA & $\bullet$ \\
\hline Saurida gracilis Quoy \& Gaimard, 1824 & $\mathrm{~S}$ & IWP & $\bullet$ \\
\hline Saurida tumbil (Bloch, 1795) & $\mathrm{S}$ & IWP & $\bullet$ \\
\hline Saurida undosquamis (Richardson, 1848) & $\mathrm{S}$ & IWP & $\bullet$ \\
\hline Synodus binotatus Schultz, 1953 & $\mathrm{H} / \mathrm{S}$ & IWP & \\
\hline Synodus dermatogenys Fowler, 1912 & $\mathrm{H} / \mathrm{S}$ & IWP & \\
\hline Synodus jaculum Russell \& Cressey, 1979 & $\mathrm{H} / \mathrm{S}$ & IWP & $\bullet$ \\
\hline Synodus rubromarmoratus Russell \& Cressey, 1979 & $\mathrm{H} / \mathrm{S}$ & IWP & \\
\hline Synodus sageneus Waite, 1905 & $\mathrm{~S}$ & IWP & $\bullet$ \\
\hline Synodus variegatus (Lacépède, 1803) & $\mathrm{H} / \mathrm{S}$ & IWP & $\bullet$ \\
\hline Trachinocephalus myops (Forster, 1801) & $\mathrm{S}$ & IWP & $\bullet$ \\
\hline \multicolumn{4}{|l|}{ Family: Chanidae } \\
\hline Chanos chanos (Forsskål, 1775) & $\mathrm{S}$ & IWP & $\bullet$ \\
\hline \multicolumn{4}{|l|}{ Family: Ariidae } \\
\hline Neoarius graeffei (Kner \& Steindachner, 1867) & $\mathrm{S} / \mathrm{E} / \mathrm{FW}$ & IA & $\bullet$ \\
\hline Netuma bilineata (Valenciennes, 1840) & $\mathrm{S} / \mathrm{E}$ & IWP & $\bullet$ \\
\hline Netuma proxima (Ogilby, 1898) & $\mathrm{S}$ & IWP & $\bullet$ \\
\hline Netuma thalassina (Rüppell, 1837) & $\mathrm{S}$ & IWP & $\bullet$ \\
\hline \multicolumn{4}{|l|}{ Family: Plotosidae } \\
\hline Euristhmus microceps (Richardson, 1845) & $\mathrm{S}$ & WA & $\bullet$ \\
\hline Euristhmus nudiceps (Günther, 1880) & $\mathrm{S} / \mathrm{E}$ & IA & $\bullet$ \\
\hline Paraplotosus albilabris (Valenciennes, 1840) & $\mathrm{H} / \mathrm{S}$ & IWP & $\bullet$ \\
\hline Paraplotosus butleri Allen, 1998 & $\mathrm{H}$ & NA & $\bullet$ \\
\hline Paraplotosus muelleri (Klunzinger, 1880) & $\mathrm{H}$ & NA & $\bullet$ \\
\hline Plotosus canius Hamilton, 1822 & $\mathrm{~S} / \mathrm{E}$ & IWP & $\bullet$ \\
\hline Plotosus lineatus (Thunberg, 1791) & $\mathrm{H} / \mathrm{E}$ & IWP & $\bullet$ \\
\hline \multicolumn{4}{|l|}{ Family: Batrachoididae } \\
\hline Batrachomoeus dahli (Rendahl, 1922) & $\mathrm{H}$ & WA & $\bullet$ \\
\hline Batrachomoeus occidentalis Hutchins, 1976 & $\mathrm{H} / \mathrm{S}$ & A & $\bullet$ \\
\hline Batrachomoeus trispinosus (Günther, 1861) & $\mathrm{H}$ & IWP & $\bullet$ \\
\hline Halophryne diemensis (Lesueur, 1824) & $\mathrm{H}$ & IWP & $\bullet$ \\
\hline Halophryne ocellatus Hutchins, 1974 & $\mathrm{~S}$ & WA & $\bullet$ \\
\hline \multicolumn{4}{|l|}{ Family: Gobiesocidae } \\
\hline Diademichthys lineatus (Sauvage, 1883) & $\mathrm{H}$ & IWP & \\
\hline Discotrema crinophila Briggs, 1976 & $\mathrm{H}$ & IWP & \\
\hline \multicolumn{4}{|l|}{ Family: Antennariidae } \\
\hline Antennarius analis (Schultz, 1957) & $\mathrm{H}$ & IWP & \\
\hline Antennarius biocellatus (Cuvier, 1817) & $\mathrm{E} / \mathrm{FW}$ & IWP & \\
\hline Antennarius coccineus (Lesson, 1830) & $\mathrm{H}$ & IWP & \\
\hline Antennarius commerson (Latreille, 1804) & $\mathrm{H}$ & IWP & \\
\hline Antennarius dorehensis Bleeker, 1859 & $\mathrm{H}$ & IWP & \\
\hline Antennarius hispidus (Bloch \& Schneider, 1801) & $\mathrm{H}$ & IWP & $\bullet$ \\
\hline
\end{tabular}




\begin{tabular}{|c|c|c|c|}
\hline Species & Habitat code & $\begin{array}{l}\text { Biogeographic } \\
\text { code }\end{array}$ & 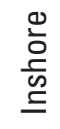 \\
\hline Antennarius maculatus (Desjardins, 1840) & $\mathrm{H}$ & IWP & \\
\hline Antennarius nummifer (Bloch \& Schneider, 1801) & $\mathrm{H}$ & IWP & - \\
\hline Antennarius pictus (Shaw \& Nodder, 1794) & $\mathrm{H}$ & IWP & - \\
\hline Histrio histrio (Linnaeus, 1758) & $\mathrm{H}$ & $\mathrm{C}$ & - \\
\hline Lophiocharon hutchinsi Pietsch, 2004 & S/SG & IA & - \\
\hline Lophiocharon trisignatus (Richardson, 1844) & $\mathrm{H}$ & IWP & - \\
\hline Tathicarpus butleri Ogilby, 1907 & $\mathrm{H}$ & IWP & - \\
\hline Tetrabrachium ocellatum Günther, 1880 & S & IWP & $\bullet$ \\
\hline \multicolumn{4}{|l|}{ Family: Ophidiidae } \\
\hline Brotula multibarbata Temminck \& Schlegel, 1847 & $\mathrm{H}$ & IWP & \\
\hline \multicolumn{4}{|l|}{ Family: Bythitidae } \\
\hline Alionematichthys piger (Alcock, 1890) & $\mathrm{H}$ & IWP & - \\
\hline Alionematichthys riukiuensis (Aoyagi, 1954) & $\mathrm{H}$ & IWP & - \\
\hline Beaglichthys bleekeri Schwarzhans \& Møller, 2007 & $\mathrm{H}$ & IA & - \\
\hline Beaglichthys macrophthalmus Machida, 1993 & $\mathrm{H} / \mathrm{S}$ & IA & - \\
\hline Brosmolus longicaudus Machida, 1993 & $\mathrm{H}$ & NA & - \\
\hline Brosmophyciops pautzkei Schultz, 1960 & $\mathrm{H}$ & IWP & \\
\hline Diancistrus alleni Schwarzhans, Møller \& Nielsen, 2005 & $\mathrm{H}$ & IWP & - \\
\hline Diancistrus beateae Schwarzhans, Møller \& Nielsen, 2005 & $\mathrm{H}$ & IWP & \\
\hline Diancistrus jeffjohnsoni Schwarzhans, Møller \& Nielsen, 2005 & $\mathrm{H}$ & NA & - \\
\hline Diancistrus novaeguineae (Machida, 1996) & $\mathrm{H}$ & IWP & - \\
\hline Didymothallus aff. criniceps Schwarzhans \& Møller, 2007 & $\mathrm{H}$ & NA & \\
\hline Didymothallus mizolepis (Günther, 1867) & $\mathrm{H}$ & NA & $\bullet$ \\
\hline Dinematichthys iluocoeteoides Bleeker, 1855 & $\mathrm{H}$ & IWP & $\bullet$ \\
\hline Dinematichthys trilobatus Møller \& Schwarzhans, 2008 & $\mathrm{H}$ & WA & $\bullet$ \\
\hline Eusurculus pistillum Schwarzhans \& Møller, 2007 & $\mathrm{H}$ & NA & $\bullet$ \\
\hline \multicolumn{4}{|l|}{ Family: Carapidae } \\
\hline Carapus mourlani (Petit, 1934) & H/EnZ & IWP & \\
\hline Encheliophis homei (Richardson, 1846) & H/EnZ & IWP & \\
\hline Onuxodon fowleri (Smith, 1955) & EnZ & IWP & - \\
\hline Onuxodon margaritiferae (Rendahl, 1921) & EnZ & A & $\bullet$ \\
\hline \multicolumn{4}{|l|}{ Family: Exocoetidae } \\
\hline Cheilopogon abei (Parin, 1996) & $\mathrm{P}$ & IWP & • \\
\hline Cheilopogon atrisignis (Jenkins, 1903) & $\mathrm{P}$ & IWP & \\
\hline Cheilopogon cyanopterus (Valenciennes, 1847) & $\mathrm{P}$ & IWP/AT & \\
\hline Cheilopogon furcatus (Mitchill, 1815) & $\mathrm{P}$ & C & \\
\hline Cypselurus hexazona (Bleeker, 1853) & $\mathrm{P}$ & IWP & \\
\hline Cypselurus naresii (Günther, 1889) & $\mathrm{P}$ & IWP & \\
\hline Cypselurus oligolepis (Bleeker, 1866) & $\mathrm{P}$ & IWP & \\
\hline Cypselurus opisthopus (Bleeker, 1866) & $\mathrm{P}$ & IWP & \\
\hline Cypselurus poecilopterus (Valenciennes, 1847) & $\mathrm{P}$ & IWP & \\
\hline Parexocoetus brachypterus (Richardson, 1846) & $\mathrm{P}$ & IWP & - \\
\hline Parexocoetus mento (Valenciennes, 1847) & $\mathrm{P}$ & IWP & $\bullet$ \\
\hline \multicolumn{4}{|l|}{ Family: Hemiramphidae } \\
\hline Arrhamphus sclerolepis Günther, 1866 & $\mathrm{P} / \mathrm{E} / \mathrm{FW}$ & IA & - \\
\hline Hemiramphus far (Forsskål, 1775) & $\mathrm{H} / \mathrm{P}$ & IWP & - \\
\hline Hemiramphus lutkei Valenciennes, 1847 & $\mathrm{P}$ & IWP & \\
\hline Hemiramphus robustus Günther, 1866 & $\mathrm{P} / \mathrm{E}$ & A & - \\
\hline Hyporhamphus affinis (Günther, 1866) & $\mathrm{P}$ & IWP & \\
\hline Hyporhamphus dussumieri (Valenciennes, 1847) & $\mathrm{H} / \mathrm{P}$ & IWP & \\
\hline
\end{tabular}




\begin{tabular}{|c|c|c|c|}
\hline Species & Habitat code & $\begin{array}{l}\text { Biogeographic } \\
\text { code }\end{array}$ & $\begin{array}{l}\text { 믐 } \\
\text { 으 }\end{array}$ \\
\hline Hyporhamphus neglectissimus Parin, Collette \& Shcherbachev, 1980 & $\mathrm{P}$ & IA & $\bullet$ \\
\hline Hyporhamphus quoyi (Valenciennes, 1847) & $\mathrm{P} / \mathrm{E}$ & IWP & $\bullet$ \\
\hline Oxyporhamphus micropterus (Valenciennes, 1847) & $\mathrm{P}$ & $\mathrm{C}$ & \\
\hline Rhynchorhamphus georgii (Valenciennes, 1847) & $\mathrm{P}$ & IWP & $\bullet$ \\
\hline Zenarchopterus buffonis (Valenciennes, 1847) & $\mathrm{P} / \mathrm{E}$ & IWP & $\bullet$ \\
\hline Zenarchopterus gilli Smith, 1945 & $\mathrm{P} / \mathrm{E}$ & IWP & $\bullet$ \\
\hline
\end{tabular}

\section{Family: Belonidae}

Ablennes hians (Valenciennes, 1846)

Platybelone argalus (Lesueur, 1821)

Strongylura incisa (Valenciennes, 1846)

Strongylura krefftii (Gunther, 1866)

Strongylura leiura (Bleeker, 1851)

Strongylura strongylura (Hasselt, 1823)

Tylosurus crocodilus (Péron \& Lesueur, 1821)

Tylosurus gavialoides (Castelnau, 1873)

Tylosurus acus (Lacepède, 1803)

$\begin{array}{ll}\mathrm{P} & \mathrm{C} \\ \mathrm{P} & \mathrm{C} \\ \mathrm{P} & \mathrm{IW} \\ \mathrm{P} / \mathrm{E} / \mathrm{FW} & \mathrm{IA} \\ \mathrm{P} & \mathrm{IW} \\ \mathrm{P} & \mathrm{IW} \\ \mathrm{P} & \mathrm{C} \\ \mathrm{P} & \mathrm{A} \\ \mathrm{P} & \mathrm{IW}\end{array}$

\section{Family: Pseudomugilidae}

Pseudomugil cyanodorsalis Allen \& Sarti, 1983

NA

\section{Family: Atherinidae}

Atherinomorus endrachtensis (Quoy \& Gaimard, 1825)

Atherinomorus lacunosus (Forster, 1801)

Atherinomorus vaigiensis (Quoy \& Gaimard, 1825)

Atherion elymus Jordan \& Starks, 1901

Craterocephalus capreoli Rendahl, 1922

Craterocephalus mugiloides (McCulloch, 1912)

Craterocephalus pauciradiatus (Günther, 1861)

Dentatherina merceri Patten \& Ivantsoff, 1983

Hypoatherina barnesi Schultz, 1953

Hypoatherina panatela (Jordan \& Richardson, 1908)

Hypoatherina temminckii (Bleeker, 1853)

Hypoatherina valenciennei (Bleeker, 1853)

\section{Family: Holocentridae}

Myripristis adusta Bleeker, 1853

Myripristis berndti Jordan \& Evermann, 1905

Myripristis hexagona (Lacépède, 1802)

Myripristis kuntee Valenciennes, 1831

Myripristis murdjan (Forsskål, 1775)

Myripristis pralinia Cuvier, 1829

Myripristis violacea Bleeker, 1851

Myripristis vittata Valenciennes, 1831

Neoniphon argenteus (Valenciennes, 1831)

Neoniphon opercularis (Valenciennes, 1831)

Neoniphon sammara (Forsskål, 1775)

Plectrypops lima (Valenciennes, 1831)

Sargocentron caudimaculatum (Rüppell, 1838)

Sargocentron cornutum (Bleeker, 1853)

Sargocentron diadema (Lacépède, 1802)

Sargocentron lepros (Allen \& Cross, 1983)

Sargocentron microstoma (Günther, 1859)

Sargocentron praslin (Lacépède, 1802)

$\begin{array}{ll}\mathrm{H} & \text { IWP } \\ \mathrm{H} & \text { IWP } \\ \mathrm{H} & \text { IWP } \\ \mathrm{H} & \text { IWP } \\ \mathrm{H} & \text { IWP } \\ \mathrm{H} & \text { IWP } \\ \mathrm{H} & \text { IWP } \\ \mathrm{H} & \text { IWP } \\ \mathrm{H} & \text { IWP } \\ \mathrm{H} & \text { IWP } \\ \mathrm{H} & \text { IWP } \\ \mathrm{H} & \text { IP } \\ \mathrm{H} & \text { IWP } \\ \mathrm{H} & \text { IWP } \\ \mathrm{H} & \text { IWP } \\ \mathrm{H} & \text { IWP } \\ \mathrm{H} & \text { IWP } \\ \mathrm{H} & \text { IWP }\end{array}$

$\begin{array}{lll}\text { IWP } & \bullet & \bullet \\ \text { IWP } & : & \\ \text { A } & & \bullet \\ \text { IWP } & \bullet & \\ \text { A } & : & \\ \text { A } & & \bullet \\ \text { WA } & & \\ \text { IWP } & \\ \text { IWP } & \\ \text { IWP } & & \\ \text { IWP } & & \end{array}$

\begin{tabular}{|c|c|}
\hline IWP & - \\
\hline IWP & - \\
\hline IWP & $\bullet \quad \bullet$ \\
\hline IWP & - \\
\hline IWP & - $\bullet$ \\
\hline IWP & $\bullet$ \\
\hline IWP & • \\
\hline IWP & $\bullet$ \\
\hline IWP & • \\
\hline IWP & • \\
\hline IWP & • \\
\hline IP & $\bullet$ \\
\hline IWP & $\bullet \quad \bullet$ \\
\hline $\begin{array}{l}\text { IWP } \\
\text { IWP }\end{array}$ & • \\
\hline IWP & $\bullet$ \\
\hline IWP & $\bullet$ \\
\hline IWP & $\bullet$ \\
\hline
\end{tabular}




\begin{tabular}{|c|c|c|c|}
\hline Species & Habitat code & $\begin{array}{l}\text { Biogeographic } \\
\text { code }\end{array}$ & 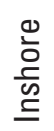 \\
\hline Sargocentron punctatissimum (Cuvier, 1829) & $\mathrm{H}$ & IWP & \\
\hline Sargocentron rubrum (Forsskål, 1775) & $\mathrm{H}$ & $\mathrm{IWP} / \mathrm{AT}$ & $\bullet$ \\
\hline Sargocentron spiniferum (Forsskål, 1775) & $\mathrm{H}$ & IWP & \\
\hline Sargocentron tiere (Cuvier, 1829) & $\mathrm{H}$ & IWP & \\
\hline Sargocentron tiereoides (Bleeker, 1853) & $\mathrm{H}$ & IWP & \\
\hline Sargocentron violaceum (Bleeker, 1853) & $\mathrm{H}$ & IWP & \\
\hline
\end{tabular}

Family: Aulostomidae

Aulostomus chinensis (Linnaeus, 1766)

$\mathrm{H} / \mathrm{P}$

IWP

Family: Fistulariidae

Fistularia commersonii Rüppell, 1838

$\mathrm{P}$

C

Fistularia petimba Lacépède, 1803

Family: Centriscidae

Centriscus cristatus (De Vis, 1885)

Centriscus scutatus Linnaeus, 1758

S

S

IWP

Family: Solenostomidae

Solenostomus halimeda Orr, Fritzsche \& Randall, 2002

Solenostomus paradoxus (Pallas, 1770)

$\begin{array}{ll}\mathrm{H} & \text { IW } \\ \mathrm{H} / \mathrm{S} & \text { IWP }\end{array}$

\section{Family: Syngnathidae}

Bhanotia fasciolata (Duméril, 1870)

Choeroichthys brachysoma (Bleeker, 1855)

Choeroichthys latispinosus Dawson, 1978

Corythoichthys amplexus Dawson \& Randall, 1975

Corythoichthys flavofasciatus (Rüppell, 1838)

Corythoichthys haematopterus (Bleeker, 1851)

Corythoichthys intestinalis (Ramsay, 1881)

Corythoichthys schultzi Herald, 1953

Cosmocampus banneri (Herald \& Randall, 1972)

Doryrhamphus excisus Kaup, 1856

Doryrhamphus janssi (Herald \& Randall, 1972)

Doryrhamphus negrosensis Herre, 1934

Dunckerocampus dactyliophorus (Bleeker, 1853)

Filicampus tigris (Castelnau, 1879)

Halicampus brocki (Herald, 1953)

Halicampus dunckeri (Chabanaud, 1929)

Halicampus nitidus (Günther, 1873)

Haliichthys taeniophorus Gray, 1859

Hippichthys cyanospilos (Bleeker, 1854)

Hippichthys penicillus (Cantor, 1850)

Hippocampus angustus Günther, 1870

Hippocampus kampylotrachelos Bleeker, 1854

Hippocampus planifrons Peters, 1877

Micrognathus andersonii (Bleeker, 1858)

Micrognathus brevirostris (Rüppell, 1838)

Micrognathus micronotopterus (Fowler, 1938)

Minyichthys myersi (Herald \& Randall, 1972)

Syngnathoides biaculeatus (Bloch, 1785)
$\mathrm{H}$

H/SG

$\mathrm{H}$

$\mathrm{H}$

$\mathrm{H}$

$\mathrm{H}$

$\mathrm{H}$

$\mathrm{H}$

$\mathrm{H}$

$\mathrm{H}$

$\mathrm{H}$

$\mathrm{H}$

$\mathrm{H}$

S

$\mathrm{H}$

$\mathrm{H}$

$\mathrm{H}$

$\mathrm{S}$

$\mathrm{E} / \mathrm{M}$

E/SG

$\mathrm{H}$

H/S/E

$\mathrm{H}$

H/SG

$\mathrm{H}$

H/SG

$\mathrm{H}$

H/SG

H

$\mathrm{H}$
IWP / AT

IWP

IWP

IWP

IWP

IWP

WA

IWP

IWP

IWP

IWP

IWP

IWP

IWP

IWP

IWP

IWP

A

IWP

IWP

IWP

IA

IWP

IWP

WA

IA

WA

IWP

IWP

IWP

IWP

IWP

\section{Family: Apistidae}

Apistus carinatus (Bloch \& Schneider, 1801)

Cheroscorpaena tridactyla Mees, 1964
$\mathrm{H}$

H
IWP

IA 


\begin{tabular}{|c|c|c|c|}
\hline Species & Habitat code & $\begin{array}{l}\text { Biogeographic } \\
\text { code }\end{array}$ & $\begin{array}{l}\stackrel{0}{\circ} \\
\frac{D}{\mathscr{c}} \\
\stackrel{5}{\subseteq}\end{array}$ \\
\hline \multicolumn{4}{|l|}{ Family: Scorpaenidae } \\
\hline Dendrochirus biocellatus (Fowler, 1938) & $\mathrm{H}$ & IWP & \\
\hline Dendrochirus brachypterus (Cuvier, 1829) & $\mathrm{H}$ & IWP & \\
\hline Dendrochirus zebra (Cuvier, 1829) & $\mathrm{H}$ & IWP & $\bullet$ \\
\hline Parascorpaena mcadamsi (Fowler, 1938) & $\mathrm{H}$ & IWP & \\
\hline Parascorpaena mossambica (Peters, 1855) & $\mathrm{H}$ & IWP & \\
\hline Parascorpaena picta (Kuhl \& Hasselt, 1829) & $\mathrm{H}$ & IWP & $\bullet$ \\
\hline Pterois antennata (Bloch, 1787) & $\mathrm{H}$ & IWP & $\bullet$ \\
\hline Pterois radiata Cuvier, 1829 & $\mathrm{H}$ & IWP & \\
\hline Pterois volitans (Linnaeus, 1758) & $\mathrm{H}$ & IWP & $\bullet$ \\
\hline Scorpaenodes albaiensis (Evermann \& Seale, 1907) & $\mathrm{H}$ & IWP & \\
\hline Scorpaenodes guamensis Quoy \& Gaimard, 1824 & $\mathrm{H}$ & IWP & \\
\hline Scorpaenodes hirsutus (Smith, 1957) & $\mathrm{H}$ & IWP & \\
\hline Scorpaenodes kelloggi (Jenkins, 1903) & $\mathrm{H}$ & IWP & \\
\hline Scorpaenodes minor (Smith, 1958) & $\mathrm{H}$ & IWP & \\
\hline Scorpaenodes parvipinnis (Garrett, 1864) & $\mathrm{H}$ & IWP & \\
\hline Scorpaenodes varipinnis Smith, 1957 & $\mathrm{H}$ & IWP & \\
\hline Scorpaenopsis diabolus (Cuvier, 1829) & $\mathrm{H}$ & IWP & $\bullet$ \\
\hline Scorpaenopsis furneauxi Whitley, 1959 & $\mathrm{~S}$ & NA & \\
\hline Scorpaenopsis macrochir Ogilby, 1910 & $\mathrm{H} / \mathrm{S}$ & IWP & \\
\hline Scorpaenopsis neglecta Heckel, 1837 & S & IWP & $\bullet$ \\
\hline Scorpaenopsis oxycephala (Bleeker, 1849) & $\mathrm{H}$ & IWP & \\
\hline Scorpaenopsis papuensis Cuvier, 1829 & $\mathrm{H}$ & IWP & \\
\hline Scorpaenopsis possi Randall \& Eschmeyer, 2001 & $\mathrm{H}$ & IWP & \\
\hline Scorpaenopsis venosa (Cuvier, 1829) & $\mathrm{H}$ & IWP & $\bullet$ \\
\hline Scorpaenopsis vittapinna Randall \& Eschmeyer, 2001 & $\mathrm{H}$ & IWP & \\
\hline Sebastapistes cyanostigma (Bleeker, 1856) & $\mathrm{H}$ & IWP & \\
\hline Sebastapistes fowleri (Pietschmann, 1934) & $\mathrm{H}$ & IWP & \\
\hline Sebastapistes strongia (Cuvier, 1829) & $\mathrm{H}$ & IWP & \\
\hline Sebastapistes tinkhami (Fowler, 1946) & $\mathrm{H}$ & IWP & \\
\hline Taenianotus triacanthus Lacépède, 1802 & $\mathrm{H}$ & $\mathrm{IP}$ & \\
\hline \multicolumn{4}{|l|}{ Family: Synanceiidae } \\
\hline Erosa daruma (Whitley, 1932) & $\mathrm{H}$ & WA & $\bullet$ \\
\hline Inimicus didactylus (Pallas, 1769) & $\mathrm{S}$ & IWP & $\bullet$ \\
\hline Inimicus sinensis (Valenciennes, 1833) & $\mathrm{S}$ & IWP & $\bullet$ \\
\hline Synanceia horrida (Linnaeus, 1766) & $\mathrm{H}$ & IWP & $\bullet$ \\
\hline Synanceia verrucosa Bloch \& Schneider, 1801 & $\mathrm{H}$ & IWP & \\
\hline \multicolumn{4}{|l|}{ Family: Tetrarogidae } \\
\hline Ablabys taenianotus (Cuvier, 1829) & $\mathrm{H}$ & IWP & \\
\hline Cottapistus cottoides (Linnaeus, 1758) & S & IWP & $\bullet$ \\
\hline Liocranium pleurostigma (Weber, 1913) ${ }^{c}$ & $\mathrm{~S}$ & IA & $\bullet$ \\
\hline Paracentropogon longispinis (Cuvier, 1829) ${ }^{\mathrm{d}}$ & $\mathrm{S}$ & IWP & \\
\hline Paracentropogon vespa Ogilby, $1910^{\mathrm{d}}$ & $\mathrm{H} / \mathrm{S}$ & NA & $\bullet$ \\
\hline \multicolumn{4}{|l|}{ Family: Caracanthidae } \\
\hline Caracanthus maculatus (Gray, 1831) & $\mathrm{H}$ & IWP & \\
\hline Caracanthus unipinna (Gray, 1831) & $\mathrm{H}$ & IWP & \\
\hline \multicolumn{4}{|l|}{ Family: Aploactinidae } \\
\hline Paraploactis pulvinus Poss \& Eschmeyer, 1978 & S & NA & $\bullet$ \\
\hline
\end{tabular}




\begin{tabular}{|c|c|c|c|}
\hline Species & Habitat code & $\begin{array}{l}\text { Biogeographic } \\
\text { code }\end{array}$ & 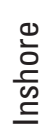 \\
\hline \multicolumn{4}{|l|}{ Family: Platycephalidae } \\
\hline Cymbacephalus bosschei (Bleeker, 1860) & $\mathrm{H} / \mathrm{S}$ & IWP & $\bullet$ \\
\hline Cymbacephalus nematophthalmus (Günther, 1860) & $\mathrm{S} / \mathrm{E}$ & IWP & $\bullet$ \\
\hline Cymbacephalus staigeri (Castelnau, 1875) & $\mathrm{S}$ & IWP & \\
\hline Elates ransonnettii (Steindachner, 1877) & $\mathrm{S}$ & IWP & $\bullet$ \\
\hline Inegocia harrisii (McCulloch, 1914) & S & NA & $\bullet$ \\
\hline Inegocia japonica (Tilesius, 1812) & $\mathrm{S}$ & IWP & $\bullet$ \\
\hline Platycephalus endrachtensis Quoy \& Gaimard, 1825 & $\mathrm{~S} / \mathrm{E}$ & IA & $\bullet$ \\
\hline Platycephalus n. sp (cf. indicus) (Imamura, in press) & $\mathrm{H} / \mathrm{E}$ & NA & $\bullet$ \\
\hline Suggrundus macracanthus (Bleeker, 1869) & $\mathrm{S}$ & IWP & \\
\hline Sunagocia arenicola (Schultz, 1966) & $\mathrm{H} / \mathrm{S}$ & IWP & \\
\hline Sunagocia otaitensis (Cuvier, 1829) & $\mathrm{S}$ & IWP & \\
\hline Thysanophrys celebica (Bleeker, 1854) & $\mathrm{H} / \mathrm{S}$ & IWP & \\
\hline Thysanophrys chiltonae Schultz, 1966 & $\mathrm{H} / \mathrm{S}$ & IWP & \\
\hline Thysanophrys papillolabium Schultz, 1966 & $\mathrm{~S}$ & IWP & \\
\hline \multicolumn{4}{|l|}{ Family: Pegasidae } \\
\hline Pegasus volitans Linnaeus, 1758 & $\mathrm{~S} / \mathrm{E}$ & IWP & $\bullet$ \\
\hline \multicolumn{4}{|l|}{ Family: Ambassidae } \\
\hline Ambassis gymnocephalus (Lacépède, 1802) & $\mathrm{S}$ & IWP & $\bullet$ \\
\hline Ambassis interrupta Bleeker, 1852 & S & IA & $\bullet$ \\
\hline Ambassis macleayi (Castelnau, 1878) & S & IA & $\bullet$ \\
\hline Ambassis nalua (Hamilton, 1822) & $\mathrm{S}$ & IWP & $\bullet$ \\
\hline Ambassis vachellii Richardson, 1846 & $\mathrm{~S}$ & IWP & $\bullet$ \\
\hline \multicolumn{4}{|l|}{ Family: Latidae } \\
\hline Lates calcarifer (Bloch, 1790) & $\mathrm{P} / \mathrm{E}$ & IWP & $\bullet$ \\
\hline Psammoperca waigiensis (Cuvier, 1828) & $\mathrm{H}$ & IWP & $\bullet$ \\
\hline \multicolumn{4}{|l|}{ Family: Centrogeniidae } \\
\hline Centrogenys vaigiensis (Quoy \& Gaimard, 1824) & $\mathrm{H}$ & IWP & $\bullet$ \\
\hline \multicolumn{4}{|l|}{ Family: Serranidae } \\
\hline Aethaloperca rogaa (Forsskål, 1775) & $\mathrm{H}$ & IWP & $\bullet$ \\
\hline Anyperodon leucogrammicus (Valenciennes, 1828) & $\mathrm{H}$ & IWP & $\bullet$ \\
\hline Aporops bilinearis Schultz, 1943 & $\mathrm{H}$ & IWP & \\
\hline Belonoperca chabanaudi Fowler \& Bean, 1930 & $\mathrm{H}$ & IWP & \\
\hline Cephalopholis argus Bloch \& Schneider, 1801 & $\mathrm{H}$ & IWP & $\bullet$ \\
\hline Cephalopholis boenak (Bloch, 1790) & $\mathrm{H}$ & IWP & $\bullet$ \\
\hline Cephalopholis cyanostigma (Valenciennes, 1828) & $\mathrm{H}$ & IWP & $\bullet$ \\
\hline Cephalopholis leopardus (Lacépède, 1801) & $\mathrm{H}$ & IWP & $\bullet$ \\
\hline Cephalopholis microprion (Bleeker, 1852) & $\mathrm{H}$ & IWP & \\
\hline Cephalopholis miniata (Forsskål, 1775) & $\mathrm{H}$ & IWP & \\
\hline Cephalopholis sexmaculata (Rüppell, 1830) & $\mathrm{H}$ & IWP & \\
\hline Cephalopholis spiloparaea (Valenciennes, 1828) & $\mathrm{H}$ & IWP & \\
\hline Cephalopholis urodeta (Forster, 1801) & $\mathrm{H}$ & IWP & \\
\hline Chromileptes altivelis (Valenciennes, 1828) & $\mathrm{H}$ & IWP & $\bullet$ \\
\hline Epinephelus areolatus (Forsskål, 1775) & $\mathrm{H}$ & IWP & $\bullet$ \\
\hline Epinephelus bilobatus Randall \& Allen, 1987 & $\mathrm{H} / \mathrm{S}$ & NA & $\bullet$ \\
\hline Epinephelus bleekeri (Vaillant, 1878) & $\mathrm{H}$ & IWP & $\bullet$ \\
\hline Epinephelus coeruleopunctatus (Bloch, 1790) & $\mathrm{H}$ & IWP & $\bullet$ \\
\hline Epinephelus coioides (Hamilton, 1822) & $\mathrm{H} / \mathrm{E}$ & IWP & $\bullet$ \\
\hline Epinephelus corallicola (Valenciennes, 1828) & $\mathrm{H} / \mathrm{E}$ & IWP & $\bullet$ \\
\hline
\end{tabular}




\begin{tabular}{|c|c|c|c|}
\hline Species & Habitat code & $\begin{array}{l}\text { Biogeographic } \\
\text { code }\end{array}$ & $\begin{array}{l}\frac{0}{0} \\
\frac{c}{\mathscr{D}} \\
\stackrel{5}{\Xi}\end{array}$ \\
\hline Epinephelus fasciatus (Forsskål, 1775) & $\mathrm{H}$ & IWP & $\bullet$ \\
\hline Epinephelus fuscoguttatus (Forsskål, 1775) & $\mathrm{H}$ & IWP & \\
\hline Epinephelus hexagonatus (Bloch \& Schneider, 1801) & $\mathrm{H}$ & IWP & \\
\hline Epinephelus lanceolatus (Bloch, 1790) & $\mathrm{H}$ & IWP & $\bullet$ \\
\hline Epinephelus macrospilos (Bleeker, 1855) & $\mathrm{H}$ & IWP & \\
\hline Epinephelus maculatus (Bloch, 1790) & $\mathrm{H}$ & IWP & \\
\hline Epinephelus malabaricus (Bloch \& Schneider, 1801) & $\mathrm{H} / \mathrm{E}$ & IWP & - \\
\hline Epinephelus melanostigma Schultz, 1953 & $\mathrm{H}$ & IWP & \\
\hline Epinephelus merra Bloch, 1793 & $\mathrm{H}$ & IWP & \\
\hline Epinephelus multinotatus (Peters, 1876) & $\mathrm{H}$ & IO & - \\
\hline Epinephelus ongus (Bloch, 1790) & $\mathrm{H}$ & IWP & • \\
\hline Epinephelus polyphekadion (Bleeker, 1849) & $\mathrm{H}$ & IWP & - \\
\hline Epinephelus quoyanus (Valenciennes, 1830) & $\mathrm{H}$ & IWP & - \\
\hline Epinephelus rivulatus (Valenciennes, 1830) & $\mathrm{H}$ & IWP & - \\
\hline Epinephelus sexfasciatus (Valenciennes, 1828) & S & IWP & $\bullet$ \\
\hline Epinephelus spilotoceps Schultz, 1953 & $\mathrm{H}$ & IWP & \\
\hline Epinephelus tauvina Forsskål, 1775 & $\mathrm{H}$ & IWP & $\bullet$ \\
\hline Epinephelus tukula Morgans, 1959 & $\mathrm{H}$ & IWP & \\
\hline Gracila albomarginata (Fowler \& Bean, 1930) & $\mathrm{H}$ & IWP & \\
\hline Grammistes sexlineatus (Thünberg, 1792) & $\mathrm{H}$ & IWP & \\
\hline Grammistops ocellatus Schultz, 1953 & $\mathrm{H}$ & IWP & \\
\hline Liopropoma susumi (Jordan \& Seale, 1906) & $\mathrm{H}$ & IWP & - \\
\hline Plectranthias longimanus Weber, 1913 & $\mathrm{H}$ & IWP & \\
\hline Plectropomus areolatus (Rüppell, 1830) & $\mathrm{H}$ & IWP & \\
\hline Plectropomus laevis (Lacépède, 1801) & $\mathrm{H}$ & IWP & \\
\hline Plectropomus leopardus (Lacépède, 1802) & $\mathrm{H}$ & IWP & \\
\hline Plectropomus maculatus (Bloch, 1790) & $\mathrm{H}$ & IWP & - \\
\hline Plectropomus oligacanthus (Bleeker, 1854) & $\mathrm{H}$ & IWP & \\
\hline Pseudanthias dispar (Herre, 1955) & $\mathrm{H}$ & IWP & \\
\hline Pseudanthias huchtii (Bleeker, 1857) & $\mathrm{H}$ & IWP & \\
\hline Pseudanthias lori (Lubbock \& Randall, 1976) & $\mathrm{H}$ & IWP & \\
\hline Pseudanthias pascalus (Jordan \& Tanaka, 1927) & $\mathrm{H}$ & IWP & \\
\hline Pseudanthias pleurotaenia (Bleeker, 1857) & $\mathrm{H}$ & IWP & \\
\hline Pseudanthias sheni Randall \& Allen, 1989 & $\mathrm{H}$ & WA & \\
\hline Pseudanthias squamipinnis (Peters, 1855) & $\mathrm{H}$ & IWP & \\
\hline Pseudanthias tuka (Herre \& Montalban, 1927) & $\mathrm{H}$ & IWP & \\
\hline Pseudogramma polyacantha (Bleeker, 1856) & $\mathrm{H}$ & IWP & \\
\hline Rainfordia opercularis McCulloch, 1923 & $\mathrm{H}$ & NA & - \\
\hline Variola albimarginata Baissac, 1953 & $\mathrm{H}$ & IWP & \\
\hline Variola louti (Forsskål, 1775) & $\mathrm{H}$ & IWP & \\
\hline \multicolumn{4}{|l|}{ Family: Pseudochromidae } \\
\hline Amsichthys knighti (Allen, 1987) & $\mathrm{H}$ & IWP & \\
\hline Assiculoides desmonotus Gill \& Hutchins, 1997 & $\mathrm{H} / \mathrm{S} / \mathrm{SG}$ & WA & • \\
\hline Assiculus punctatus Richardson, 1846 & $\mathrm{H}$ & NA & • \\
\hline Blennodesmus scapularis Günther, 1872 & $\mathrm{H}$ & IWP & - \\
\hline Congrogadus spinifer (Borodin, 1933) & $\mathrm{H}$ & NA & - \\
\hline Congrogadus subducens (Richardson, 1843) & $\mathrm{H}$ & IWP & - \\
\hline Labracinus cyclophthalmus (Müller \& Troschel, 1849) & $\mathrm{H}$ & IWP & - \\
\hline Labracinus lineatus (Castelnau, 1875) & $\mathrm{H}$ & WA & - \\
\hline Lubbockichthys multisquamatus (Allen, 1987) & $\mathrm{H}$ & IWP & \\
\hline Manonichthys splendens (Fowler, 1931) & $\mathrm{H}$ & IO & \\
\hline Pictichromis paccagnellae (Axelrod, 1973) & $\mathrm{H}$ & IWP & \\
\hline
\end{tabular}




\begin{tabular}{|c|c|c|c|c|}
\hline Species & Habitat code & $\begin{array}{l}\text { Biogeographic } \\
\text { code }\end{array}$ & 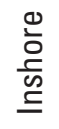 & $\begin{array}{l}\frac{\omega}{2} \\
\frac{0}{\omega} \\
\text { 志 }\end{array}$ \\
\hline Pseudochromis andamanensis Lubbock, 1980 & $\mathrm{H}$ & $\mathrm{IO}$ & & • \\
\hline Pseudochromis bitaeniatus (Fowler, 1931) & $\mathrm{H}$ & IWP & & - \\
\hline Pseudochromis cyanotaenia Bleeker, 1857 & $\mathrm{H}$ & IWP & & - \\
\hline Pseudochromis fuscus Müller \& Troschel, 1849 & $\mathrm{H}$ & IWP & - & - \\
\hline Pseudochromis howsoni Allen, 1995 & $\mathrm{H}$ & WA & & - \\
\hline Pseudochromis marshallensis Schultz, 1953 & $\mathrm{H}$ & IWP & & - \\
\hline Pseudochromis tapeinosoma Bleeker, 1853 & $\mathrm{H}$ & IWP & & - \\
\hline Pseudochromis wilsoni (Whitley, 1929) & $\mathrm{H}$ & NA & - & - \\
\hline Pseudoplesiops rosae Schultz, 1943 & $\mathrm{H}$ & IWP & & - \\
\hline Pseudoplesiops typus Bleeker, 1858 & $\mathrm{H}$ & IWP & & - \\
\hline \multicolumn{5}{|l|}{ Family: Grammistidae } \\
\hline Diploprion bifasciatum Cuvier, 1828 & $\mathrm{H}$ & IWP & $\bullet$ & \\
\hline \multicolumn{5}{|l|}{ Family: Plesiopidae } \\
\hline Acanthoplesiops indicus (Day, 1888) & $\mathrm{H}$ & IWP & & - \\
\hline Calloplesiops altivelis (Steindachner, 1903) & $\mathrm{H}$ & IWP & - & \\
\hline Notograptus guttatus Günther, 1867 & $\mathrm{H}$ & IA & - & - \\
\hline Plesiops coeruleolineatus Rüppell, 1835 & $\mathrm{H}$ & IWP & & - \\
\hline Plesiops gracilis Mooi \& Randall, 1991 & $\mathrm{H}$ & IWP & & - \\
\hline Plesiops verecundus Mooi, 1995 & $\mathrm{H}$ & IWP & & - \\
\hline Steeneichthys plesiopsus Allen \& Randall, 1985 & $\mathrm{H}$ & IWP & & $\bullet$ \\
\hline \multicolumn{5}{|l|}{ Family: Acanthoclinidae } \\
\hline Belonepterygion fasciolatum (Ogilby, 1889) & $\mathrm{H}$ & IWP & $\bullet$ & \\
\hline \multicolumn{5}{|l|}{ Family: Glaucosomatidae } \\
\hline Glaucosoma magnificum (Ogilby, 1915) & $\mathrm{H}$ & $\mathrm{A}$ & $\bullet$ & \\
\hline \multicolumn{5}{|l|}{ Family: Terapontidae } \\
\hline Amniataba caudavittata (Richardson, 1845) & $\mathrm{M} / \mathrm{FW}$ & IA & - & \\
\hline Pelates quadrilineatus (Bloch, 1790) & E/SG & IWP & - & \\
\hline Pelates sexlineatus (Quoy \& Gaimard, 1825) ${ }^{\mathrm{e}}$ & E/SG & A & $\bullet$ & \\
\hline Terapon jarbua (Forsskål, 1775) & $\mathrm{S} / \mathrm{M} / \mathrm{FW}$ & IWP & - & - \\
\hline Terapon puta Cuvier, 1829 & $\mathrm{~S} / \mathrm{M} / \mathrm{FW}$ & IWP & - & \\
\hline Terapon theraps Cuvier, 1829 & $\mathrm{~S} / \mathrm{M}$ & IWP & $\bullet$ & $\bullet$ \\
\hline \multicolumn{5}{|l|}{ Family: Kuhliidae } \\
\hline Kuhlia mugil (Forster, 1801) & $\mathrm{H} / \mathrm{P}$ & IP & & $\bullet$ \\
\hline \multicolumn{5}{|l|}{ Family: Priacanthidae } \\
\hline Heteropriacanthus cruentatus (Lacépède, 1801) & $\mathrm{H}$ & $\mathrm{C}$ & - & - \\
\hline Priacanthus hamrur (Forsskål, 1775) & $\mathrm{H}$ & IWP & & • \\
\hline Priacanthus macracanthus Cuvier, 1829 & $\mathrm{H} / \mathrm{S}$ & IWP & & • \\
\hline Priacanthus tayenus Richardson, 1846 & $\mathrm{H}$ & IWP & $\bullet$ & \\
\hline \multicolumn{5}{|l|}{ Family: Apogonidae } \\
\hline Apogon caudicinctus Randall \& Smith, 1988 & $\mathrm{H} / \mathrm{S}$ & IWP & & $\bullet$ \\
\hline Apogon crassiceps Garman, 1903 & $\mathrm{H}$ & IWP & $\bullet$ & $\bullet$ \\
\hline Apogon cf. doryssa (Jordan \& Seale, 1906) & $\mathrm{H}$ & IWP & & $\bullet$ \\
\hline Apogon talboti (Smith, 1961) & $\mathrm{H}$ & IWP & $\bullet$ & $\bullet$ \\
\hline Apogon unicolor (Steindachner \& Döderlein, 1883) & $\mathrm{H}$ & IWP & • & \\
\hline Apogonichthyoides atripes (Cuvier, 1828) $\mathrm{f}$ & $\mathrm{H} / \mathrm{S}$ & IWP & • & \\
\hline Apogonichthyoides brevicaudatus ( Weber, 1909) & $\mathrm{S}$ & IA & - & \\
\hline Apogonichthyoides melas (Bleeker, 1848) & $\mathrm{H}$ & IWP & & - \\
\hline Apogonichthyoides timorensis (Bleeker, 1854) & $\mathrm{H}$ & IWP & - & - \\
\hline Apogonichthys ocellatus (Weber, 1913) & $\mathrm{H}$ & IWP & & . \\
\hline
\end{tabular}




\begin{tabular}{|c|c|c|c|}
\hline Species & Habitat code & $\begin{array}{l}\text { Biogeographic } \\
\text { code }\end{array}$ & 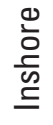 \\
\hline Apogonichthys perdix Bleeker, 1854 & $\mathrm{H}$ & IWP & \\
\hline Cercamia eremia (Allen, 1987) & $\mathrm{H}$ & IWP & \\
\hline Cheilodipterus artus Smith, 1961 & $\mathrm{H}$ & IWP & \\
\hline Cheilodipterus isostigma (Schultz, 1940) & $\mathrm{H}$ & IWP & \\
\hline Cheilodipterus macrodon (Lacépède, 1802) & $\mathrm{H}$ & IWP & - \\
\hline Cheilodipterus quinquelineatus Cuvier, 1828 & $\mathrm{H}$ & IWP & - \\
\hline Cheilodipterus singapurensis Bleeker, 1860 & $\mathrm{H}$ & IWP & \\
\hline Fibramia amboinensis Bleeker, 1853 & $\mathrm{~S}$ & IWP & - \\
\hline Fibramia lateralis (Valenciennes, 1832) & $\mathrm{S}$ & IWP & - \\
\hline Fibramia thermalis (Cuvier, 1829) & $\mathrm{H}$ & IWP & \\
\hline Foa fo Jordan \& Seale, $1905^{\circ}$ & $\mathrm{H}$ & IWP & \\
\hline Foa hyalina (Smith \& Radcliffe, 1912) & $\mathrm{H}$ & IWP & \\
\hline Fowleria aurita (Valenciennes, 1831) & $\mathrm{H}$ & IWP & $\bullet$ \\
\hline Fowleria isostigma (Jordan \& Seale, 1906) & $\mathrm{H}$ & IWP & $\bullet$ \\
\hline Fowleria marmorata (Alleyne \& Macleay, 1877) & $\mathrm{H}$ & IWP & \\
\hline Fowleria vaiulae (Jordan \& Seale, 1906) & $\mathrm{H}$ & IWP & \\
\hline Fowleria variegata (Valenciennes, 1832) & $\mathrm{H}$ & IWP & \\
\hline Gymnapogon аппопа (Whitley, 1936) & $\mathrm{H}$ & NA & - \\
\hline Gymnapogon philippinus (Herre, 1939) & $\mathrm{H}$ & IWP & - \\
\hline Gymnapogon urospilotus Lachner, $1953^{\text {h }}$ & $\mathrm{H}$ & IWP & - \\
\hline Gymnapogon vanderbilti (Fowler, 1938) & $\mathrm{H}$ & IWP & \\
\hline Jaydia poecilopterus (Cuvier, 1828) & $\mathrm{S}$ & IWP & - \\
\hline Neamia octospina Smith \& Radcliffe, 1912 & $\mathrm{H}$ & IWP & \\
\hline Nectamia bandanensis (Bleeker, 1854) & $\mathrm{H}$ & IA & \\
\hline Nectamia fusca (Quoy \& Gaimard, 1825) & $\mathrm{H}$ & IWP & - \\
\hline Nectamia savayensis (Günther, 1872) & $\mathrm{H}$ & IWP & \\
\hline Ostorhinchus angustatus (Smith \& Radcliffe, 1911) & $\mathrm{H}$ & IWP & \\
\hline Ostorhinchus aureus (Lacépède, 1802) & $\mathrm{H}$ & IWP & \\
\hline Ostorhinchus cavitensis (Jordan \& Seale, 1907) & $\mathrm{S}$ & IWP & - \\
\hline Ostorhinchus chrysopomus (Bleeker, 1854) & $\mathrm{H}$ & IWP & \\
\hline Ostorhinchus chrysotaenia (Bleeker, 1851) & $\mathrm{H}$ & IWP & \\
\hline Ostorhinchus compressus (Smith \& Radcliffe, 1911) & $\mathrm{H}$ & IWP & \\
\hline Ostorhinchus cookii (Macleay, 1881) & $\mathrm{H}$ & IWP & - \\
\hline Ostorhinchus cyanosoma (Bleeker, 1853) & $\mathrm{H}$ & IWP & - \\
\hline Ostorhinchus doederleini (Jordan \& Snyder, 1901) & $\mathrm{H}$ & IWP & - \\
\hline Ostorhinchus endekataenia (Bleeker, 1852) & $\mathrm{H}$ & IWP & - \\
\hline Ostorhinchus fasciatus (Shaw, 1790) & $\mathrm{S}$ & IWP & - \\
\hline Ostorhinchus fleurieu Lacépède, 1802 & $\mathrm{H}$ & IWP & \\
\hline Ostorhinchus hartzfeldii (Bleeker, 1852) & $\mathrm{H}$ & IWP & \\
\hline Ostorhinchus nigrofasciatus (Lachner, 1953) & $\mathrm{H}$ & IWP & \\
\hline Ostorhinchus novemfasciatus (Cuvier, 1828) & $\mathrm{H}$ & IWP & \\
\hline Ostorhinchus ocellicaudus (Allen, Kuiter \& Randall 1994) & $\mathrm{H}$ & IWP & \\
\hline Ostorhinchus pallidofasciatus (Allen, 1987) & $\mathrm{H}$ & NA & - \\
\hline Ostorhinchus rueppellii (Günther, 1859) & $\mathrm{H}$ & IA & - \\
\hline Ostorhinchus sealei (Fowler, 1918) & $\mathrm{H}$ & IWP & \\
\hline Ostorhinchus semilineatus (Temminck \& Schlegel, 1843) & $\mathrm{H}$ & IWP & \\
\hline Ostorhinchus taeniophorus (Regan, 1908) & $\mathrm{H}$ & IWP & \\
\hline Prisitapogon unitaeniatus (Allen, 1995) & $\mathrm{H}$ & NA & - \\
\hline Pristiapogon exostigma (Jordan \& Starks, 1906) & $\mathrm{H}$ & IWP & \\
\hline Pristiapogon fraenatus (Valenciennes, 1832) & $\mathrm{H}$ & IWP & - \\
\hline Pristiapogon kallopterus (Bleeker, 1856) & $\mathrm{H}$ & IWP & \\
\hline Pristicon rhodopterus (Bleeker, 1852) & $\mathrm{H}$ & IWP & \\
\hline
\end{tabular}




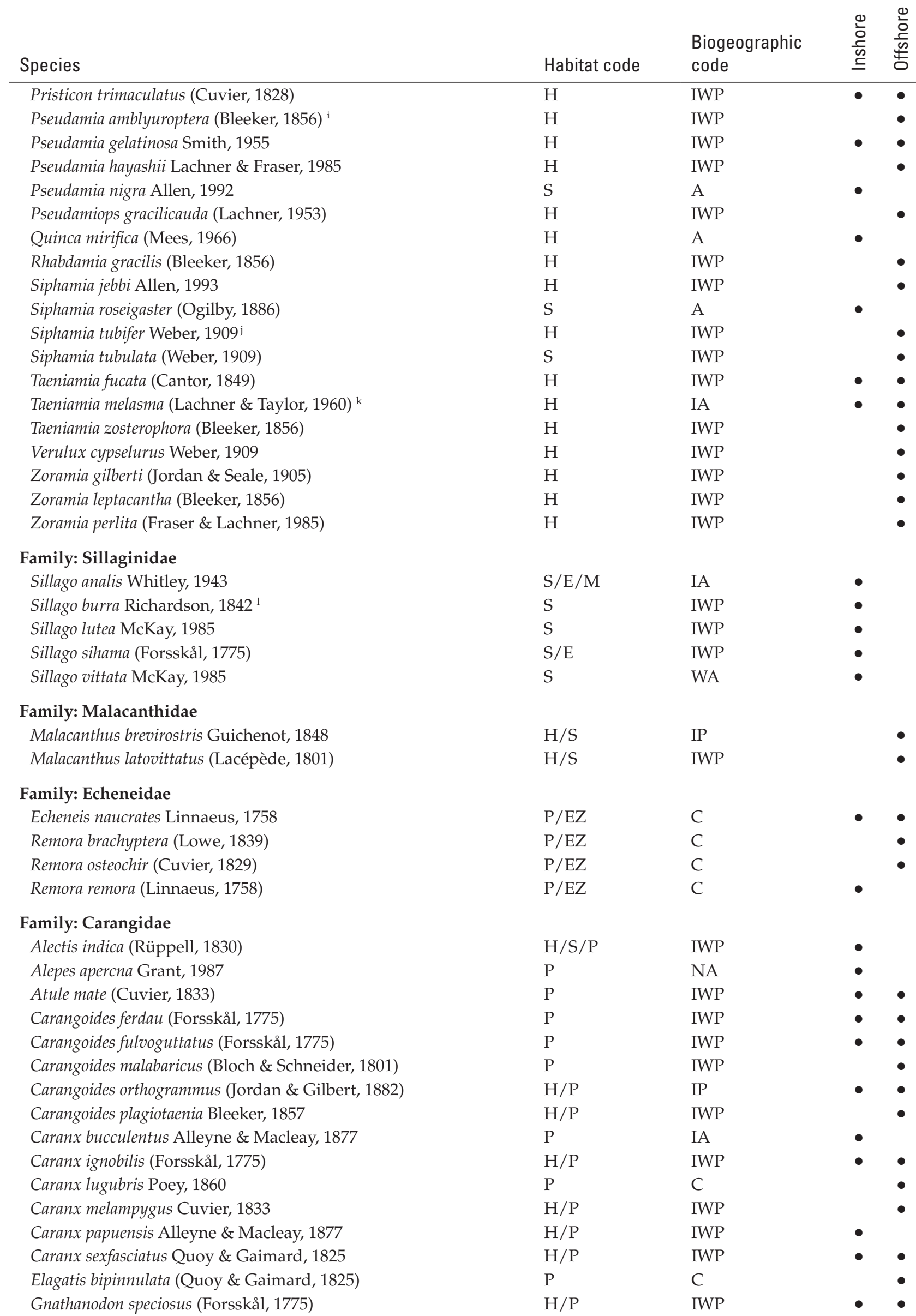




\begin{tabular}{|c|c|c|c|}
\hline Species & Habitat code & $\begin{array}{l}\text { Biogeographic } \\
\text { code }\end{array}$ & $\frac{a}{a}$ \\
\hline Megalaspis cordyla (Linnaeus, 1758) & $\mathrm{P}$ & IWP & $\bullet$ \\
\hline Pantolabus radiatus (Macleay, 1881 ) & $\mathrm{P}$ & IA & - \\
\hline Parastromateus niger (Bloch, 1795) & $\mathrm{P}$ & IWP & - \\
\hline Scomberoides commersonnianus Lacépède, 1801 & $\mathrm{H} / \mathrm{P}$ & IWP & - \\
\hline Scomberoides lysan (Forsskål, 1775) & $\mathrm{P}$ & IWP & - \\
\hline Scomberoides tala (Cuvier, 1832) & $\mathrm{P}$ & IWP & • \\
\hline Scomberoides tol (Cuvier, 1832) & $\mathrm{P}$ & IWP & \\
\hline Selar boops (Cuvier, 1833) & $\mathrm{P}$ & IWP & • \\
\hline Selar crumenophthalmus (Bloch, 1793) & $\mathrm{P}$ & $\mathrm{C}$ & $\bullet$ \\
\hline Selaroides leptolepis (Cuvier, 1833) & $\mathrm{P}$ & IWP & $\bullet$ \\
\hline Seriola dumerili (Risso, 1810) & $\mathrm{P}$ & $\mathrm{C}$ & \\
\hline Seriola rivoliana Valenciennes, 1833 & $\mathrm{P}$ & $\mathrm{C}$ & \\
\hline Trachinotus baillonii (Lacépède, 1801) & $\mathrm{P}$ & IWP & - \\
\hline Trachinotus blochii (Lacépède, 1801) & $\mathrm{H} / \mathrm{P}$ & IWP & \\
\hline Trachinotus botla (Shaw, 1803) & $\mathrm{P}$ & IWP & • \\
\hline Ulua mentalis (Cuvier, 1833) & $\mathrm{P}$ & IWP & $\bullet$ \\
\hline \multicolumn{4}{|l|}{ Family: Coryphaenidae } \\
\hline Coryphaena hippurus Linnaeus, 1758 & $\mathrm{P}$ & $\mathrm{C}$ & $\bullet$ \\
\hline \multicolumn{4}{|l|}{ Family: Leiognathidae } \\
\hline Equulites leuciscus (Günther, 1860) & $\mathrm{S}$ & IWP & - \\
\hline Eubleekeria splendens (Cuvier, 1829) & $\mathrm{S} / \mathrm{M}$ & IWP & - \\
\hline Gazza rhombea Kimura, Yamashita \& Iwatsuki, 2000 & $\mathrm{~S}$ & IWP & - \\
\hline Leiognathus equulus (Forsskål, 1775) & $\mathrm{S} / \mathrm{E}$ & IWP & - \\
\hline Nuchequula gerreoides (Bleeker, 1851) ${ }^{\mathrm{m}}$ & $\mathrm{S} / \mathrm{E} / \mathrm{M}$ & IWP & • \\
\hline Photopectoralis bindus (Valenciennes, 1835) & $\mathrm{S}$ & IWP & - \\
\hline Secutor ruconius (Hamilton, 1822) ${ }^{\mathrm{n}}$ & S/E & IWP & $\bullet$ \\
\hline \multicolumn{4}{|l|}{ Family: Lutjanidae } \\
\hline Aphareus furca (Lacépède, 1801) & $\mathrm{H}$ & IWP & \\
\hline Aphareus rutilans Cuvier, 1830 & $\mathrm{H}$ & IWP & \\
\hline Aprion virescens Valenciennes, 1830 & $\mathrm{H}$ & IWP & \\
\hline Lutjanus argentimaculatus (Forsskål, 1775) & $\mathrm{H} / \mathrm{E} / \mathrm{M} / \mathrm{FW}$ & IWP & - \\
\hline Lutjanus biguttatus (Valenciennes, 1830) & $\mathrm{H}$ & IWP & \\
\hline Lutjanus bohar (Forsskål, 1775) & $\mathrm{H}$ & IWP & - \\
\hline Lutjanus carponotatus (Richardson, 1842) & $\mathrm{H}$ & IWP & - \\
\hline Lutjanus decussatus (Cuvier, 1828) & $\mathrm{H}$ & IWP & - \\
\hline Lutjanus erythropterus Bloch, 1790 & $\mathrm{~S}$ & IWP & - \\
\hline Lutjanus fulviflamma (Forsskål, 1775) & $\mathrm{H}$ & IWP & - \\
\hline Lutjanus fulvus (Bloch \& Schneider, 1801) & $\mathrm{H} / \mathrm{M}$ & IWP & \\
\hline Lutjanus gibbus (Forsskål, 1775) & $\mathrm{H}$ & IWP & \\
\hline Lutjanus johnii (Bloch, 1792) & $\mathrm{H} / \mathrm{E} / \mathrm{M}$ & IWP & $\bullet$ \\
\hline Lutjanus kasmira (Forsskål, 1775) & $\mathrm{H}$ & IWP & \\
\hline Lutjanus lemniscatus (Valenciennes, 1828) & $\mathrm{H}$ & IWP & $\bullet$ \\
\hline Lutjanus lutjanus Bloch, 1790 & $\mathrm{H}$ & IWP & $\bullet$ \\
\hline Lutjanus malabaricus (Bloch \& Schneider, 1801) & $\mathrm{H} / \mathrm{S}$ & IWP & $\bullet$ \\
\hline Lutjanus monostigma (Cuvier, 1828) & $\mathrm{H}$ & IWP & \\
\hline Lutjanus quinquelineatus (Bloch, 1790) & $\mathrm{H}$ & IWP & - \\
\hline Lutjanus rivulatus (Cuvier, 1828) & $\mathrm{H}$ & IWP & • \\
\hline Lutjanus rufolineatus (Valenciennes, 1830) & $\mathrm{H}$ & IWP & \\
\hline Lutjanus russellii (Bleeker, 1849) & $\mathrm{H}$ & IWP & $\bullet$ \\
\hline Lutjanus sebae (Cuvier, 1816) & $\mathrm{H} / \mathrm{M}$ & IWP & - \\
\hline Lutjanus vitta (Quoy \& Gaimard, 1824) & $\mathrm{H}$ & IWP & . \\
\hline
\end{tabular}




\begin{tabular}{|c|c|c|c|}
\hline Species & Habitat code & $\begin{array}{l}\text { Biogeographic } \\
\text { code }\end{array}$ & 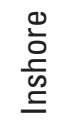 \\
\hline Macolor macularis Fowler, 1931 & $\mathrm{H}$ & IWP & \\
\hline Macolor niger (Forsskål, 1775) & $\mathrm{H}$ & IWP & - \\
\hline Paracaesio xanthura (Bleeker, 1869) & $\mathrm{H}$ & IWP & \\
\hline Symphorichthys spilurus (Günther, 1874) & $\mathrm{H} / \mathrm{S}$ & IWP & \\
\hline Symphorus nematophorus (Bleeker, 1860) & $\mathrm{H}$ & IWP & $\bullet$ \\
\hline \multicolumn{4}{|l|}{ Family: Caesionidae } \\
\hline Caesio caerulaurea Lacépède, 1801 & $\mathrm{H} / \mathrm{P}$ & IWP & - \\
\hline Caesio cuning (Bloch, 1791) & $\mathrm{H} / \mathrm{P}$ & IWP & - \\
\hline Caesio lunaris Cuvier, 1830 & $\mathrm{H} / \mathrm{P}$ & IWP & \\
\hline Caesio teres Seale, 1906 & $\mathrm{H} / \mathrm{P}$ & IWP & \\
\hline Caesio xanthonotus Bleeker, 1853 & $\mathrm{P}$ & IWP & \\
\hline Pterocaesio digramma (Bleeker, 1865) & $\mathrm{H} / \mathrm{P}$ & IWP & \\
\hline Pterocaesio pisang (Bleeker, 1853) & $\mathrm{H}$ & IWP & \\
\hline Pterocaesio randalli Carpenter, 1987 & $\mathrm{H} / \mathrm{P}$ & IWP & \\
\hline Pterocaesio tile (Cuvier, 1830) & $\mathrm{H} / \mathrm{P}$ & IWP & \\
\hline Pterocaesio trilineata Carpenter, 1987 & $\mathrm{H} / \mathrm{P}$ & IWP & \\
\hline \multicolumn{4}{|l|}{ Family: Nemipteridae } \\
\hline Nemipterus hexodon (Quoy \& Gaimard, 1824) & S & IWP & $\bullet$ \\
\hline Pentapodus emeryii (Richardson, 1843) & $\mathrm{H} / \mathrm{S}$ & IWP & - \\
\hline Pentapodus paradiseus (Günther, 1859) & $\mathrm{H}$ & IWP & - \\
\hline Pentapodus porosus (Valenciennes, 1830) & $\mathrm{H}$ & IA & - \\
\hline Scaevius milii (Bory de Saint-Vincent, 1823) & $\mathrm{H} / \mathrm{S}$ & NA & - \\
\hline Scolopsis affinis Peters, 1877 & $\mathrm{H} / \mathrm{S}$ & IWP & \\
\hline Scolopsis bilineata (Bloch, 1793) & $\mathrm{H}$ & IWP & - \\
\hline Scolopsis lineata Quoy \& Gaimard, 1824 & $\mathrm{H} / \mathrm{S}$ & IWP & \\
\hline Scolopsis margaritifera (Cuvier, 1830) & $\mathrm{H} / \mathrm{S}$ & IWP & \\
\hline Scolopsis monogramma (Cuvier, 1830) & $\mathrm{H} / \mathrm{S}$ & IWP & $\bullet$ \\
\hline Scolopsis taeniopterus (Cuvier, 1830) & $\mathrm{H} / \mathrm{S}$ & IWP & - \\
\hline Scolopsis trilineata Kner, 1868 & $\mathrm{H} / \mathrm{S}$ & IWP & \\
\hline Scolopsis xenochrous Günther, 1872 & $\mathrm{H} / \mathrm{S}$ & IWP & \\
\hline \multicolumn{4}{|l|}{ Family: Gerreidae } \\
\hline Gerres filamentosus Cuvier, 1829 & $\mathrm{~S} / \mathrm{E}$ & IWP & $\bullet$ \\
\hline Gerres oyena (Forsskål, 1775) & $\mathrm{S} / \mathrm{E} / \mathrm{M}$ & IWP & $\bullet$ \\
\hline Gerres subfasciatus Cuvier, 1830 & $\mathrm{~S} / \mathrm{E}$ & A & $\bullet$ \\
\hline \multicolumn{4}{|l|}{ Family: Haemulidae } \\
\hline Diagramma pictum (Thunberg, 1792) & $\mathrm{H}$ & IA & - \\
\hline Plectorhinchus chaetodonoides Lacépède, 1801 & $\mathrm{H}$ & IWP & $\bullet$ \\
\hline Plectorhinchus gibbosus (Lacépède, 1802) & $\mathrm{H} / \mathrm{E}$ & IWP & - \\
\hline Plectorhinchus lessonii (Cuvier, 1830) & $\mathrm{H}$ & IWP & \\
\hline Plectorhinchus lineatus (Linnaeus, 1758) & $\mathrm{H}$ & IWP & \\
\hline Plectorhinchus multivittatus (Macleay, 1878) & $\mathrm{H}$ & IWP & $\bullet$ \\
\hline Plectorhinchus pica (Cuvier, 1830) & $\mathrm{H}$ & IWP & \\
\hline Plectorhinchus polytaenia (Bleeker, 1852) & $\mathrm{H}$ & IWP & $\bullet$ \\
\hline Plectorhinchus schotaf (Forsskål, 1775) & $\mathrm{H}$ & IWP & - \\
\hline Plectorhinchus vittatus (Linnaeus, 1758) & $\mathrm{H}$ & IWP & \\
\hline Pomadasys argenteus (Forsskål, 1775) & $\mathrm{S} / \mathrm{E}$ & IWP & - \\
\hline Pomadasys kaakan (Cuvier, 1830) & $\mathrm{S} / \mathrm{E}$ & IWP & - \\
\hline Pomadasys maculatus (Bloch, 1793) & $\mathrm{S} / \mathrm{E}$ & IWP & $\bullet$ \\
\hline \multicolumn{4}{|l|}{ Family: Lethrinidae } \\
\hline Gnathodentex aureolineatus (Lacépède, 1802) & $\mathrm{H}$ & IWP & \\
\hline
\end{tabular}




\begin{tabular}{|c|c|c|c|}
\hline Species & Habitat code & $\begin{array}{l}\text { Biogeographic } \\
\text { code }\end{array}$ & 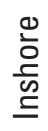 \\
\hline Gymnocranius grandoculis (Valenciennes, 1830) & $\mathrm{H} / \mathrm{S}$ & IWP & \\
\hline Gymnocranius griseus (Temminck \& Schlegel, 1843) & $\mathrm{S}$ & IWP & \\
\hline Gymnocranius microdon (Bleeker, 1851) & $\mathrm{H} / \mathrm{S}$ & IWP & \\
\hline Lethrinus atkinsoni Seale, 1910 & $\mathrm{H}$ & IWP & $\bullet$ \\
\hline Lethrinus erythracanthus Valenciennes, 1830 & $\mathrm{H}$ & IWP & \\
\hline Lethrinus erythropterus Valenciennes, 1830 & $\mathrm{H}$ & IWP & \\
\hline Lethrinus harak (Forsskål, 1775) & $\mathrm{H} / \mathrm{S}$ & IWP & \\
\hline Lethrinus laticaudis Alleyne \& Macleay, 1877 & $\mathrm{H} / \mathrm{S}$ & IWP & • \\
\hline Lethrinus lentjan (Lacépède, 1802) & $\mathrm{H} / \mathrm{S}$ & IWP & • \\
\hline Lethrinus microdon Valenciennes, 1830 & $\mathrm{H} / \mathrm{S}$ & IWP & \\
\hline Lethrinus miniatus (Forster, 1801) & $\mathrm{H}$ & IWP & \\
\hline Lethrinus nebulosus (Forsskål, 1775) & $\mathrm{H} / \mathrm{S}$ & IWP & • \\
\hline Lethrinus obsoletus (Forsskål, 1775) & $\mathrm{H} / \mathrm{S}$ & IWP & \\
\hline Lethrinus olivaceus Valenciennes, 1830 & $\mathrm{H}$ & IWP & • \\
\hline Lethrinus rubrioperculatus Sato, 1978 & $\mathrm{H} / \mathrm{S}$ & IWP & \\
\hline Lethrinus semicinctus Valenciennes, 1830 & $\mathrm{H} / \mathrm{S}$ & IWP & \\
\hline Lethrinus xanthochilus Klunzinger, 1870 & $\mathrm{H}$ & IWP & \\
\hline Monotaxis grandoculis (Forsskål, 1775) & $\mathrm{H}$ & IWP & \\
\hline \multicolumn{4}{|l|}{ Family: Sparidae } \\
\hline Acanthopagrus latus (Houttuyn, 1782) & $\mathrm{S} / \mathrm{E} / \mathrm{M}$ & IWP & $\bullet$ \\
\hline Acanthopagrus palmaris (Whitley, 1935) & $\mathrm{S} / \mathrm{E}$ & NA & • \\
\hline \multicolumn{4}{|l|}{ Family: Sciaenidae } \\
\hline Austronibea oedogenys Trewavas, 1977 & $\mathrm{~S} / \mathrm{E}$ & IA & $\bullet$ \\
\hline Johnius amblycephalus (Bleeker, 1855) & $\mathrm{S} / \mathrm{E}$ & IWP & $\bullet$ \\
\hline Johnius borneensis (Bleeker, 1851) & $\mathrm{S} / \mathrm{E}$ & IWP & $\bullet$ \\
\hline Johnius laevis Sasaki \& Kailola, 1991 & S & IA & $\bullet$ \\
\hline Johnius novaeguineae (Nichols, 1950) & $\mathrm{S} / \mathrm{E}$ & IA & $\bullet$ \\
\hline Nibea microgenys Sasaki, 1992 & $\mathrm{~S} / \mathrm{E}$ & NA & $\bullet$ \\
\hline Otolithes ruber (Bloch \& Schneider, 1801) & $\mathrm{S}$ & IWP & $\bullet$ \\
\hline \multicolumn{4}{|l|}{ Family: Mullidae } \\
\hline Mulloidichthys flavolineatus (Lacépède, 1801) & $\mathrm{H} / \mathrm{S}$ & IWP & \\
\hline Mulloidichthys vanicolensis (Valenciennes, 1831) & $\mathrm{H} / \mathrm{S}$ & IWP & \\
\hline Parupeneus barberinoides (Bleeker, 1852) & $\mathrm{H} / \mathrm{S}$ & IWP & \\
\hline Parupeneus barberinus (Lacépède, 1801) & $\mathrm{H} / \mathrm{S}$ & IWP & \\
\hline Parupeneus crassilabris (Valenciennes, 1831) & $\mathrm{H} / \mathrm{S}$ & IWP & \\
\hline Parupeneus cyclostomus (Lacépède, 1801) & $\mathrm{H} / \mathrm{S}$ & IWP & \\
\hline Parupeneus indicus (Shaw, 1803) & $\mathrm{H} / \mathrm{S}$ & IWP & • \\
\hline Parupeneus multifasciatus (Quoy \& Gaimard, 1825) & $\mathrm{H} / \mathrm{S}$ & IWP & \\
\hline Parupeneus pleurostigma (Bennett, 1831) & $\mathrm{H} / \mathrm{S}$ & IWP & \\
\hline Upeneus tragula Richardson, 1846 & $\mathrm{H} / \mathrm{S} / \mathrm{E}$ & IWP & $\bullet$ \\
\hline \multicolumn{4}{|l|}{ Family: Monodactylidae } \\
\hline Monodactylus argenteus (Linnaeus, 1758) & $\mathrm{E}$ & IWP & \\
\hline \multicolumn{4}{|l|}{ Family: Leptobramidae } \\
\hline Leptobrama muelleri Steindachner, 1878 & $\mathrm{~S} / \mathrm{P}$ & IA & $\bullet$ \\
\hline \multicolumn{4}{|l|}{ Family: Pempheridae } \\
\hline Parapriacanthus ransonneti Steindachner, 1870 & $\mathrm{H} / \mathrm{P}$ & IWP & \\
\hline Pempheris mangula Cuvier, 1829 & $\mathrm{H}$ & IWP & $\bullet$ \\
\hline Pempheris oualensis Cuvier, 1831 & $\mathrm{H}$ & IWP & • \\
\hline Pempheris ypsilychnus Mooi \& Jubb, 1996 & $\mathrm{H}$ & NA & $\bullet$ \\
\hline
\end{tabular}




\begin{tabular}{|c|c|c|c|}
\hline Species & Habitat code & $\begin{array}{l}\text { Biogeographic } \\
\text { code }\end{array}$ & 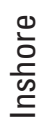 \\
\hline \multicolumn{4}{|l|}{ Family: Toxotidae } \\
\hline Toxotes chatareus (Hamilton, 1822) & $\mathrm{S} / \mathrm{E} / \mathrm{M} / \mathrm{FW}$ & IWP & - \\
\hline Toxotes jaculatrix (Pallas, 1767) & $\mathrm{S} / \mathrm{E} / \mathrm{M} / \mathrm{FW}$ & IWP & $\bullet$ \\
\hline \multicolumn{4}{|l|}{ Family: Kyphosidae } \\
\hline Kyphosus bigibbus Lacépède, 1801 & $\mathrm{H}$ & IWP & \\
\hline Kyphosus cinerascens (Forsskål, 1775) & $\mathrm{H}$ & IWP & \\
\hline Kyphosus vaigiensis (Quoy \& Gaimard, 1825) ㅇ & $\mathrm{H}$ & IWP & - \\
\hline \multicolumn{4}{|l|}{ Family: Ephippidae } \\
\hline Drepane punctata (Linnaeus, 1758) & S & IWP & $\bullet$ \\
\hline Platax batavianus Cuvier, 1831 & $\mathrm{H} / \mathrm{S}$ & IWP & • \\
\hline Platax orbicularis (Forsskål, 1775) & $\mathrm{H}$ & IWP & - \\
\hline Platax pinnatus (Linnaeus, 1758) & $\mathrm{H}$ & IWP & \\
\hline Platax teira (Forsskål, 1775) & $\mathrm{H}$ & IWP & - \\
\hline Zabidius novemaculeatus (McCulloch, 1916) & S & IA & $\bullet$ \\
\hline \multicolumn{4}{|l|}{ Family: Scatophagidae } \\
\hline Scatophagus argus (Linnaeus, 1766) & $\mathrm{E} / \mathrm{M} / \mathrm{FW}$ & IWP & - \\
\hline Selenotoca multifasciata (Richardson, 1846) & $\mathrm{E} / \mathrm{M} / \mathrm{FW}$ & IA & $\bullet$ \\
\hline \multicolumn{4}{|l|}{ Family: Rhinoprenidae } \\
\hline Rhinoprenes pentanemus Munro, 1964 & $\mathrm{~S}$ & IA & $\bullet$ \\
\hline \multicolumn{4}{|l|}{ Family: Chaetodontidae } \\
\hline Chaetodon adiergastos Seale, 1910 & $\mathrm{H}$ & IWP & $\bullet$ \\
\hline Chaetodon aureofasciatus Macleay, 1878 & $\mathrm{H}$ & IA & - \\
\hline Chaetodon auriga Forsskål, 1775 & $\mathrm{H}$ & IWP & - \\
\hline Chaetodon baronessa Cuvier, 1829 & $\mathrm{H}$ & IWP & \\
\hline Chaetodon bennetti Cuvier, 1831 & $\mathrm{H}$ & IWP & \\
\hline Chaetodon citrinellus Cuvier, 1831 & $\mathrm{H}$ & IWP & \\
\hline Chaetodon decussatus Cuvier, 1829 & $\mathrm{H}$ & IWP & \\
\hline Chaetodon ephippium Cuvier, 1831 & $\mathrm{H}$ & IWP & \\
\hline Chaetodon kleinii Bloch, 1790 & $\mathrm{H}$ & IWP & \\
\hline Chaetodon lineolatus Cuvier, 1831 & $\mathrm{H}$ & IWP & - \\
\hline Chaetodon lunula (Lacépède, 1802) & $\mathrm{H}$ & IWP & - \\
\hline Chaetodon lunulatus Quoy \& Gaimard, 1825 & $\mathrm{H}$ & IWP & \\
\hline Chaetodon melannotus Bloch \& Schneider, 1801 & $\mathrm{H}$ & IWP & \\
\hline Chaetodon meyeri Bloch \& Schneider, 1801 & $\mathrm{H}$ & IWP & \\
\hline Chaetodon ocellicaudus Cuvier, 1831 & $\mathrm{H}$ & IWP & \\
\hline Chaetodon ornatissimus Cuvier, 1831 & $\mathrm{H}$ & IWP & \\
\hline Chaetodon oxycephalus Bleeker, 1853 & $\mathrm{H}$ & IWP & \\
\hline Chaetodon pelewensis Kner, 1868 & $\mathrm{H}$ & IWP & \\
\hline Chaetodon punctatofasciatus Cuvier, 1831 & $\mathrm{H}$ & IWP & \\
\hline Chaetodon rafflesii Bennett, 1830 & $\mathrm{H}$ & IWP & \\
\hline Chaetodon semeion Bleeker, 1855 & $\mathrm{H}$ & IWP & \\
\hline Chaetodon speculum Cuvier, 1831 & $\mathrm{H}$ & IWP & - \\
\hline Chaetodon trifascialis Quoy \& Gaimard, 1825 & $\mathrm{H}$ & IWP & - \\
\hline Chaetodon ulietensis Cuvier, 1831 & $\mathrm{H}$ & IWP & \\
\hline Chaetodon unimaculatus Bloch, 1787 & $\mathrm{H}$ & IWP & \\
\hline Chaetodon vagabundus Linnaeus, 1758 & $\mathrm{H}$ & IWP & - \\
\hline Chelmon marginalis Richardson, 1842 & $\mathrm{H}$ & $\mathrm{A}$ & - \\
\hline Chelmon muelleri Klunzinger, 1879 & $\mathrm{H}$ & $\mathrm{A}$ & $\bullet$ \\
\hline Coradion altivelis McCulloch, 1916 & $\mathrm{H}$ & IWP & \\
\hline
\end{tabular}




\begin{tabular}{|c|c|c|c|}
\hline Species & Habitat code & $\begin{array}{l}\text { Biogeographic } \\
\text { code }\end{array}$ & $\begin{array}{l}\text { ఏ } \\
\text { 음 } \\
\text { 号 }\end{array}$ \\
\hline Coradion chrysozonus (Cuvier, 1831) & $\mathrm{H}$ & IWP & $\bullet$ \\
\hline Forcipiger flavissimus Jordan \& McGregor, 1898 & $\mathrm{H}$ & IWP & \\
\hline Forcipiger longirostris (Broussonet, 1782) & $\mathrm{H}$ & IWP & \\
\hline Hemitaurichthys polylepis (Bleeker, 1857) & $\mathrm{H}$ & IWP & \\
\hline Heniochus acuminatus (Linnaeus, 1758) & $\mathrm{H}$ & IWP & $\bullet$ \\
\hline Heniochus chrysostomus Cuvier, 1831 & $\mathrm{H}$ & IWP & \\
\hline Heniochus monoceros Cuvier, 1831 & $\mathrm{H}$ & IWP & \\
\hline Heniochus singularius Smith \& Radcliffe, 1911 & $\mathrm{H}$ & IWP & \\
\hline Heniochus varius (Cuvier, 1829) & $\mathrm{H}$ & IWP & \\
\hline Parachaetodon ocellatus (Cuvier, 1831) & $\mathrm{H}$ & IWP & $\bullet$ \\
\hline \multicolumn{4}{|l|}{ Family: Pomacanthidae } \\
\hline Apolemichthys trimaculatus (Cuvier, 1831) & $\mathrm{H}$ & IWP & \\
\hline Centropyge bicolor (Bloch, 1787) & $\mathrm{H}$ & IWP & \\
\hline Centropyge bispinosa (Günther, 1860) & $\mathrm{H}$ & IWP & \\
\hline Centropyge eibli Klausewitz, 1963 & $\mathrm{H}$ & IA & \\
\hline Centropyge fisheri (Snyder, 1904) & $\mathrm{H}$ & $\mathrm{IP}$ & \\
\hline Centropyge nox (Bleeker, 1853) & $\mathrm{H}$ & IWP & \\
\hline Centropyge tibicen (Cuvier, 1831) & $\mathrm{H}$ & IWP & \\
\hline Centropyge vrolikii (Bleeker, 1853) & $\mathrm{H}$ & IWP & \\
\hline Chaetodontoplus duboulayi (Günther, 1867) & $\mathrm{H} / \mathrm{S}$ & IWP & $\bullet$ \\
\hline Chaetodontoplus mesoleucus (Bloch, 1787) & $\mathrm{H}$ & IWP & $\bullet$ \\
\hline Chaetodontoplus personifer (McCulloch, 1914) & $\mathrm{H}$ & NA & $\bullet$ \\
\hline Genicanthus lamarck (Lacépède, 1802) & $\mathrm{H}$ & IWP & \\
\hline Pomacanthus imperator (Bloch, 1787) & $\mathrm{H}$ & IWP & \\
\hline Pomacanthus navarchus (Cuvier, 1831) & $\mathrm{H}$ & IWP & \\
\hline Pomacanthus semicirculatus (Cuvier, 1831) & $\mathrm{H}$ & IWP & \\
\hline Pomacanthus sexstriatus (Cuvier, 1831) & $\mathrm{H}$ & IWP & $\bullet$ \\
\hline Pomacanthus xanthometopon (Bleeker, 1853) & $\mathrm{H}$ & IWP & \\
\hline Pygoplites diacanthus (Boddaert, 1772) & $\mathrm{H}$ & IWP & \\
\hline \multicolumn{4}{|l|}{ Family: Pomacentridae } \\
\hline Abudefduf bengalensis (Bloch, 1787) & $\mathrm{H}$ & IWP & $\bullet$ \\
\hline Abudefduf septemfasciatus (Cuvier, 1830) & $\mathrm{H}$ & IWP & \\
\hline Abudefduf sexfasciatus (Lacépède, 1801) & $\mathrm{H}$ & IWP & \\
\hline Abudefduf sordidus (Forsskål, 1775) & $\mathrm{H}$ & IWP & $\bullet$ \\
\hline Abudefduf vaigiensis (Quoy \& Gaimard, 1825) & $\mathrm{H}$ & IWP & $\bullet$ \\
\hline Acanthochromis polyacanthus (Bleeker, 1855) & $\mathrm{H}$ & IWP & $\bullet$ \\
\hline Amblyglyphidodon aureus (Cuvier, 1830) & $\mathrm{H}$ & IWP & \\
\hline Amblyglyphidodon batunai Allen, 1995 & $\mathrm{H}$ & IA & $\bullet$ \\
\hline Amblyglyphidodon curacao (Bloch, 1787) & $\mathrm{H}$ & IWP & \\
\hline Amblyglyphidodon leucogaster (Bleeker, 1847) & $\mathrm{H}$ & IWP & \\
\hline Amphiprion clarkii (Bennett, 1830) & $\mathrm{H} / \mathrm{EZ}$ & IWP & $\bullet$ \\
\hline Amphiprion melanopus Bleeker, 1852 & $\mathrm{H} / \mathrm{EZ}$ & IWP & \\
\hline Amphiprion ocellaris Cuvier, 1830 & $\mathrm{H} / \mathrm{EZ}$ & IWP & $\bullet$ \\
\hline Amphiprion perideraion Bleeker, 1855 & $\mathrm{H} / \mathrm{EZ}$ & IWP & \\
\hline Amphiprion rubrocinctus Richardson, 1842 & $\mathrm{H} / \mathrm{EZ}$ & NA & $\bullet$ \\
\hline Amphiprion sandaracinos Allen, 1972 & $\mathrm{H} / \mathrm{EZ}$ & IWP & $\bullet$ \\
\hline Cheiloprion labiatus (Day, 1877) & $\mathrm{H}$ & IWP & $\bullet$ \\
\hline Chromis alpha Randall, 1988 & $\mathrm{H}$ & IWP & \\
\hline Chromis amboinensis (Bleeker, 1873) & $\mathrm{H}$ & IWP & \\
\hline Chromis analis (Cuvier, 1830) & $\mathrm{H}$ & IWP & \\
\hline Chromis atripectoralis Welander \& Schultz, 1951 & $\mathrm{H}$ & IWP & $\bullet$ \\
\hline
\end{tabular}




\begin{tabular}{|c|c|c|c|}
\hline Species & Habitat code & $\begin{array}{l}\text { Biogeographic } \\
\text { code }\end{array}$ & 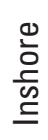 \\
\hline Chromis atripes Fowler \& Bean, 1928 & $\mathrm{H}$ & IWP & \\
\hline Chromis caudalis Randall, 1988 & $\mathrm{H}$ & IWP & \\
\hline Chromis cinerascens (Cuvier, 1830) & $\mathrm{H}$ & IWP & $\bullet$ \\
\hline Chromis elerae Fowler \& Bean, 1928 & $\mathrm{H}$ & IWP & \\
\hline Chromis fumea (Tanaka, 1917) & $\mathrm{H}$ & IWP & \\
\hline Chromis lepidolepis Bleeker, 1877 & $\mathrm{H}$ & IWP & \\
\hline Chromis lineata Fowler \& Bean, 1928 & $\mathrm{H}$ & IWP & \\
\hline Chromis margaritifer Fowler, 1946 & $\mathrm{H}$ & IWP & \\
\hline Chromis opercularis (Günther, 1867) & $\mathrm{H}$ & $\mathrm{IO}$ & \\
\hline Chromis retrofasciata Weber, 1913 & $\mathrm{H}$ & IWP & \\
\hline Chromis ternatensis (Bleeker, 1856) & $\mathrm{H}$ & IWP & \\
\hline Chromis vanderbilti (Fowler, 1941) & $\mathrm{H}$ & IWP & \\
\hline Chromis viridis (Cuvier, 1830) & $\mathrm{H}$ & IWP & $\bullet$ \\
\hline Chromis weberi Fowler \& Bean, 1928 & $\mathrm{H}$ & IWP & \\
\hline Chromis xanthochira (Bleeker, 1851) & $\mathrm{H}$ & IWP & \\
\hline Chromis xanthura (Bleeker, 1854) & $\mathrm{H}$ & IWP & \\
\hline Chrysiptera biocellata (Quoy \& Gaimard, 1825) & $\mathrm{H}$ & IWP & \\
\hline Chrysiptera brownriggii (Bennett, 1828) & $\mathrm{H}$ & IWP & \\
\hline Chrysiptera caeruleolineata (Allen, 1973) & $\mathrm{H}$ & IWP & \\
\hline Chrysiptera cyanea (Quoy \& Gaimard, 1825) & $\mathrm{H}$ & IWP & \\
\hline Chrysiptera glauca (Cuvier, 1830) & $\mathrm{H}$ & IWP & \\
\hline Chrysiptera hemicyanea (Weber, 1913) & $\mathrm{H}$ & IWP & \\
\hline Chrysiptera rex (Snyder, 1909) & $\mathrm{H}$ & IWP & \\
\hline Chrysiptera rollandi (Whitley, 1961) & $\mathrm{H}$ & IWP & $\bullet$ \\
\hline Chrysiptera talboti (Allen, 1975) & $\mathrm{H}$ & IWP & \\
\hline Chrysiptera unimaculata (Cuvier, 1830) & $\mathrm{H}$ & IWP & \\
\hline Dascyllus aruanus (Linnaeus, 1758) & $\mathrm{H}$ & IWP & \\
\hline Dascyllus melanurus Bleeker, 1854 & $\mathrm{H}$ & IWP & \\
\hline Dascyllus reticulatus (Richardson, 1846) & $\mathrm{H}$ & IWP & $\bullet$ \\
\hline Dascyllus trimaculatus (Rüppell, 1829) & $\mathrm{H}$ & IWP & $\bullet$ \\
\hline Dischistodus chrysopoecilus (Schlegel \& Müller, 1839) & $\mathrm{H}$ & IWP & \\
\hline Dischistodus darwiniensis (Whitley, 1928) & $\mathrm{H}$ & NA & $\bullet$ \\
\hline Dischistodus melanotus (Bleeker, 1858) & $\mathrm{H}$ & IWP & \\
\hline Dischistodus perspicillatus (Cuvier, 1830) & $\mathrm{H} / \mathrm{S}$ & IWP & \\
\hline Dischistodus prosopotaenia (Bleeker, 1852) & $\mathrm{H}$ & IWP & $\bullet$ \\
\hline Hemiglyphidodon plagiometopon (Bleeker, 1852) & $\mathrm{H}$ & IWP & $\bullet$ \\
\hline Lepidozygus tapeinosoma (Bleeker, 1856) & $\mathrm{H}$ & IWP & \\
\hline Neoglyphidodon melas (Cuvier, 1830) & $\mathrm{H}$ & IWP & $\bullet$ \\
\hline Neoglyphidodon nigroris (Cuvier, 1830) & $\mathrm{H}$ & IWP & $\bullet$ \\
\hline Neoglyphidodon oxyodon (Bleeker, 1858) & $\mathrm{H}$ & IWP & \\
\hline Neopomacentrus azysron (Bleeker, 1877) & $\mathrm{H}$ & IWP & $\bullet$ \\
\hline Neopomacentrus cyanomos (Bleeker, 1856) & $\mathrm{H}$ & IWP & $\bullet$ \\
\hline Neopomacentrus filamentosus (Macleay, 1882) & $\mathrm{H}$ & IWP & $\bullet$ \\
\hline Neopomacentrus taeniurus (Bleeker, 1856) & $\mathrm{H} / \mathrm{E} / \mathrm{FW}$ & IWP & $\bullet$ \\
\hline Plectroglyphidodon dickii (Liénard, 1839) & $\mathrm{H}$ & IWP & \\
\hline Plectroglyphidodon imparipennis (Vaillant \& Sauvage, 1875) & $\mathrm{H}$ & IWP & \\
\hline Plectroglyphidodon johnstonianus Fowler \& Ball, 1924 & $\mathrm{H}$ & IWP & \\
\hline Plectroglyphidodon lacrymatus (Quoy \& Gaimard, 1825) & $\mathrm{H}$ & IWP & \\
\hline Plectroglyphidodon leucozonus (Bleeker, 1859) & $\mathrm{H}$ & IWP & \\
\hline Pomacentrus adelus Allen, 1991 & $\mathrm{H}$ & IWP & $\bullet$ \\
\hline Pomacentrus alexanderae Evermann \& Seale, 1907 & $\mathrm{H}$ & IWP & \\
\hline Pomacentrus amboinensis Bleeker, 1868 & $\mathrm{H}$ & IWP & \\
\hline
\end{tabular}




\begin{tabular}{|c|c|c|c|}
\hline Species & Habitat code & $\begin{array}{l}\text { Biogeographic } \\
\text { code }\end{array}$ & 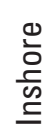 \\
\hline Pomacentrus bankanensis Bleeker, 1853 & $\mathrm{H}$ & IWP & $\bullet$ \\
\hline Pomacentrus chrysurus Cuvier, 1830 & $\mathrm{H}$ & IWP & \\
\hline Pomacentrus coelestis Jordan \& Starks, 1901 & $\mathrm{H}$ & IWP & $\bullet$ \\
\hline Pomacentrus grammorhynchus Fowler, 1918 & $\mathrm{H}$ & IWP & \\
\hline Pomacentrus lepidogenys Fowler \& Bean, 1928 & $\mathrm{H}$ & IWP & \\
\hline Pomacentrus limosus Allen, 1992 & $\mathrm{H}$ & WA & $\bullet$ \\
\hline Pomacentrus littoralis Cuvier, 1830 & $\mathrm{H}$ & IWP & $\bullet$ \\
\hline Pomacentrus milleri Taylor, 1964 & $\mathrm{H}$ & NA & $\bullet$ \\
\hline Pomacentrus moluccensis Bleeker, 1853 & $\mathrm{H}$ & IWP & \\
\hline Pomacentrus nagasakiensis Tanaka, 1917 & $\mathrm{H}$ & IWP & $\bullet$ \\
\hline Pomacentrus nigromanus Weber, 1913 & $\mathrm{H}$ & IA & $\bullet$ \\
\hline Pomacentrus nigromarginatus Allen, 1973 & $\mathrm{H}$ & IA & \\
\hline Pomacentrus pavo (Bloch, 1787) & $\mathrm{H}$ & IWP & \\
\hline Pomacentrus philippinus Evermann \& Seale, 1907 & $\mathrm{H}$ & IWP & $\bullet$ \\
\hline Pomacentrus reidi Fowler \& Bean, 1928 & $\mathrm{H}$ & IWP & \\
\hline Pomacentrus tripunctatus Cuvier, 1830 & $\mathrm{H}$ & IWP & • \\
\hline Pomacentrus vaiuli Jordan \& Seale, 1906 & $\mathrm{H}$ & IWP & \\
\hline Pomachromis richardsoni (Snyder, 1909) & $\mathrm{H}$ & IWP & \\
\hline Premnas biaculeatus (Bloch, 1790) & $\mathrm{H} / \mathrm{EZ}$ & IWP & \\
\hline Stegastes albifasciatus (Schlegel \& Müller, 1839) & $\mathrm{H}$ & IWP & \\
\hline Stegastes fasciolatus (Ogilby, 1889) & $\mathrm{H}$ & IWP & • \\
\hline Stegastes nigricans (Lacépède, 1802) & $\mathrm{H}$ & IWP & $\bullet$ \\
\hline Stegastes obreptus (Whitley, 1948) & $\mathrm{H}$ & IWP & $\bullet$ \\
\hline Stegastes punctatus (Quoy \& Gaimard, 1825) & $\mathrm{H}$ & IWP & \\
\hline \multicolumn{4}{|l|}{ Family: Cirrhitidae } \\
\hline Cirrhitichthys falco Randall, 1963 & $\mathrm{H}$ & IWP & \\
\hline Cirrhitichthys oxycephalus (Bleeker, 1855) & $\mathrm{H}$ & IWP & \\
\hline Cirrhitus pinnulatus (Bloch \& Schneider, 1801) & $\mathrm{H}$ & IWP & \\
\hline Oxycirrhites typus Bleeker, 1857 & $\mathrm{H}$ & IWP & \\
\hline Paracirrhites arcatus (Cuvier, 1829) & $\mathrm{H}$ & IWP & \\
\hline Paracirrhites forsteri (Schneider, 1801) & $\mathrm{H}$ & IWP & \\
\hline \multicolumn{4}{|l|}{ Family: Cepolidae } \\
\hline Acanthocepola abbreviata (Valeniennes, 1835) & $\mathrm{S}$ & IWP & $\bullet$ \\
\hline \multicolumn{4}{|l|}{ Family: Mugilidae } \\
\hline Ellochelon vaigiensis (Quoy \& Gaimard, 1825) & $\mathrm{H} / \mathrm{S} / \mathrm{P} / \mathrm{E}$ & IWP & $\bullet$ \\
\hline Liza alata (Steindachner, 1892) & $\mathrm{S}$ & IWP & $\bullet$ \\
\hline Liza macrolepis (Smith, 1846) ${ }^{\mathrm{n}}$ & $\mathrm{S} / \mathrm{E} / \mathrm{FW}$ & IWP & $\bullet$ \\
\hline Liza subviridis (Valenciennes, 1836) & $\mathrm{S} / \mathrm{P}$ & IWP & $\bullet$ \\
\hline Mugil cephalus Linnaeus, 1758 & $\mathrm{H} / \mathrm{S} / \mathrm{P} / \mathrm{E} / \mathrm{FW}$ & $\mathrm{C}$ & $\bullet$ \\
\hline Oedalechilus labiosus (Valenciennes, 1836) & $\mathrm{H} / \mathrm{S} / \mathrm{P} / \mathrm{E}$ & IWP & $\bullet$ \\
\hline Paramugil parmatus (Cantor, 1849) & $\mathrm{S} / \mathrm{P}$ & IWP & $\bullet$ \\
\hline Rhinomugil nasutus (De Vis, 1883) & $\mathrm{S} / \mathrm{P} / \mathrm{E}$ & IA & $\bullet$ \\
\hline Valamugil buchanani (Bleeker, 1853) & $\mathrm{H} / \mathrm{S} / \mathrm{P} / \mathrm{E}$ & IWP & $\bullet$ \\
\hline Valamugil cunnesius (Valenciennes, 1836) & $\mathrm{S} / \mathrm{P} / \mathrm{E}$ & IWP & $\bullet$ \\
\hline Valamugil seheli (Forsskål, 1775) & $\mathrm{H} / \mathrm{S} / \mathrm{P} / \mathrm{E}$ & IWP & $\bullet$ \\
\hline \multicolumn{4}{|l|}{ Family: Sphyraenidae } \\
\hline Sphyraena barracuda (Edwards, 1771) & $\mathrm{H} / \mathrm{P} / \mathrm{E}$ & $\mathrm{C}$ & $\bullet$ \\
\hline Sphyraena jello Cuvier, 1829 & $\mathrm{H} / \mathrm{P} / \mathrm{E}$ & IWP & $\bullet$ \\
\hline Sphyraena obtusata Cuvier, 1829 & $\mathrm{H} / \mathrm{P}$ & IWP & $\bullet$ \\
\hline Sphyraena putnamae Jordan \& Seale, 1907 & $\mathrm{P}$ & IWP & $\bullet$ \\
\hline
\end{tabular}




\begin{tabular}{|c|c|c|c|}
\hline Species & Habitat code & $\begin{array}{l}\text { Biogeographic } \\
\text { code }\end{array}$ & 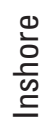 \\
\hline \multicolumn{4}{|l|}{ Family: Polynemidae } \\
\hline Eleutheronema tetradactylus (Shaw, 1804) & $\mathrm{S} / \mathrm{E}$ & IWP & - \\
\hline Parapolynemus verekeri (Saville-Kent, 1889) & $\mathrm{S} / \mathrm{E}$ & IA & - \\
\hline Polydactylus macrochir (Günther, 1867) & $\mathrm{S} / \mathrm{P} / \mathrm{E} / \mathrm{M}$ & IA & - \\
\hline Polydactylus multiradiatus (Günther, 1860) & $\mathrm{S} / \mathrm{E}$ & IA & - \\
\hline Polydactylus nigripinnis Munro, 1964 & $\mathrm{~S}$ & IA & $\bullet$ \\
\hline \multicolumn{4}{|l|}{ Family: Labridae } \\
\hline Anampses caeruleopunctatus Rüppell, 1829 & $\mathrm{H}$ & IWP & \\
\hline Anampses geographicus Valenciennes, 1840 & $\mathrm{H}$ & IWP & \\
\hline Anampses lennardi Scott, 1959 & $\mathrm{H}$ & NA & $\bullet$ \\
\hline Anampses melanurus Bleeker, 1857 & $\mathrm{H}$ & IWP & \\
\hline Anampses meleagrides Valenciennes, 1840 & $\mathrm{H}$ & IWP & \\
\hline Anampses neoguinaicus Bleeker, 1878 & $\mathrm{H}$ & IWP & \\
\hline Anampses twistii Bleeker, 1856 & $\mathrm{H}$ & IWP & \\
\hline Bodianus anthioides (Bennett, 1832) & $\mathrm{H}$ & IWP & \\
\hline Bodianus axillaris (Bennett, 1832) & $\mathrm{H}$ & IWP & $\bullet$ \\
\hline Bodianus diana (Lacépède, 1801) & $\mathrm{H}$ & IO & $\bullet$ \\
\hline Bodianus mesothorax (Bloch \& Schneider, 1801) & $\mathrm{H}$ & IWP & $\bullet$ \\
\hline Bolbometopon muricatum (Valenciennes, 1840) & $\mathrm{H}$ & IWP & \\
\hline Calotomus carolinus (Valenciennes, 1840) & $\mathrm{H}$ & IWP & \\
\hline Calotomus spinidens (Quoy \& Gaimard, 1824) & $\mathrm{H}$ & IWP & \\
\hline Cetoscarus ocellatus (Valenciennes, 1840$)^{\mathrm{q}}$ & $\mathrm{H}$ & IWP & \\
\hline Cheilinus chlorourus (Bloch, 1791) & $\mathrm{H}$ & IWP & • \\
\hline Cheilinus fasciatus (Bloch, 1791) & $\mathrm{H}$ & IWP & $\bullet$ \\
\hline Cheilinus oxycephalus Bleeker, 1853 & $\mathrm{H}$ & IWP & \\
\hline Cheilinus trilobatus Lacépède, 1801 & $\mathrm{H}$ & IWP & $\bullet$ \\
\hline Cheilinus undulatus Rüppell, 1835 & $\mathrm{H}$ & IWP & \\
\hline Cheilio inermis (Forsskål, 1775) & $\mathrm{H} / \mathrm{SG}$ & IWP & \\
\hline Chlorurus bleekeri (de Beaufort, 1940) & $\mathrm{H}$ & IWP & \\
\hline Chlorurus microrhinos (Bleeker, 1854) & $\mathrm{H}$ & IWP & \\
\hline Chlorurus sordidus (Forsskål, 1775) & $\mathrm{H}$ & IWP & $\bullet$ \\
\hline Choerodon cauteroma Gomon \& Allen, 1987 & $\mathrm{H}$ & IA & - \\
\hline Choerodon cephalotes (Castelnau, 1875) & $\mathrm{H}$ & IWP & - \\
\hline Choerodon cyanodus (Richardson, 1843) & $\mathrm{H}$ & A & - \\
\hline Choerodon monostigma Ogilby, 1910 & $\mathrm{H} / \mathrm{S}$ & IA & $\bullet$ \\
\hline Choerodon schoenleinii (Valenciennes, 1839) & $\mathrm{H}$ & IWP & $\bullet$ \\
\hline Choerodon vitta Ogilby, 1910 & $\mathrm{H}$ & IA & $\bullet$ \\
\hline Cirrhilabrus cyanopleura (Bleeker, 1851) & $\mathrm{H}$ & IWP & \\
\hline Cirrhilabrus exquisitus Smith, 1957 & $\mathrm{H}$ & IWP & \\
\hline Cirrhilabrus morrisoni Allen, 1998 & $\mathrm{H}$ & WA & \\
\hline Cirrhilabrus randalli Allen, 1995 & $\mathrm{H}$ & WA & \\
\hline Cirrhilabrus temminckii Bleeker, 1853 & $\mathrm{H}$ & IWP & \\
\hline Conniella apterygia Allen, 1983 & $\mathrm{H}$ & WA & \\
\hline Coris aygula Lacépède, 1801 & $\mathrm{H}$ & IWP & $\bullet$ \\
\hline Coris batuensis (Bleeker, 1857) & $\mathrm{H}$ & IWP & \\
\hline Coris caudimacula (Quoy \& Gaimard, 1834) & $\mathrm{H}$ & IO & $\bullet$ \\
\hline Coris dorsomacula Fowler, 1908 & $\mathrm{H}$ & IWP & \\
\hline Coris gaimard (Quoy \& Gaimard, 1824) & $\mathrm{H}$ & IWP & \\
\hline Coris pictoides Randall \& Kuiter, 1982 & $\mathrm{H}$ & IWP & • \\
\hline Cymolutes praetextatus (Quoy \& Gaimard, 1834) & $\mathrm{H} / \mathrm{S}$ & IWP & \\
\hline Diproctacanthus xanthurus (Bleeker, 1856) & $\mathrm{H}$ & IWP & $\bullet$ \\
\hline
\end{tabular}




\begin{tabular}{|c|c|c|c|}
\hline Species & Habitat code & $\begin{array}{l}\text { Biogeographic } \\
\text { code }\end{array}$ & 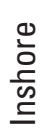 \\
\hline Epibulus insidiator (Pallas, 1770) & $\mathrm{H}$ & IWP & \\
\hline Gomphosus varius Lacépède, 1801 & $\mathrm{H}$ & IWP & $\bullet$ \\
\hline Halichoeres biocellatus Schultz, 1960 & $\mathrm{H}$ & IWP & \\
\hline Halichoeres chrysus Randall, 1981 & $\mathrm{H}$ & IWP & \\
\hline Halichoeres claudia Randall \& Rocha, 2009 & $\mathrm{H}$ & IWP & \\
\hline Halichoeres hartzfeldii (Bleeker, 1852) & $\mathrm{H} / \mathrm{S}$ & IWP & $\bullet$ \\
\hline Halichoeres hortulanus (Lacépède, 1801) & $\mathrm{H} / \mathrm{S}$ & IWP & $\bullet$ \\
\hline Halichoeres leucurus (Walbaum, 1792) & $\mathrm{H}$ & IWP & $\bullet$ \\
\hline Halichoeres margaritaceus (Valenciennes, 1839) & $\mathrm{H}$ & IWP & \\
\hline Halichoeres marginatus Rüppell, 1835 & $\mathrm{H}$ & IWP & $\bullet$ \\
\hline Halichoeres melanochir Fowler \& Bean, 1928 & $\mathrm{H}$ & IWP & $\bullet$ \\
\hline Halichoeres melanurus (Bleeker, 1851) & $\mathrm{H}$ & IWP & $\bullet$ \\
\hline Halichoeres melasmapomus Randall, 1981 & $\mathrm{H}$ & IWP & \\
\hline Halichoeres nebulosus (Valenciennes, 1839) & $\mathrm{H}$ & IWP & $\bullet$ \\
\hline Halichoeres nigrescens (Bloch \& Schneider, 1801) & $\mathrm{H}$ & IWP & $\bullet$ \\
\hline Halichoeres ornatissimus (Garrett, 1864) & $\mathrm{H}$ & IWP & \\
\hline Halichoeres prosopeion (Bleeker, 1853) & $\mathrm{H}$ & IWP & \\
\hline Halichoeres scapularis (Bennett, 1832) & $\mathrm{H} / \mathrm{S}$ & IWP & \\
\hline Halichoeres trimaculatus (Cuvier, 1834) & $\mathrm{H} / \mathrm{S}$ & IWP & \\
\hline Hemigymnus fasciatus (Bloch, 1792) & $\mathrm{H}$ & IWP & $\bullet$ \\
\hline Hemigymnus melapterus (Bloch, 1791) & $\mathrm{H}$ & IWP & $\bullet$ \\
\hline Hipposcarus longiceps (Valenciennes, 1840) & $\mathrm{H}$ & IWP & \\
\hline Hologymnosus doliatus (Lacépède, 1801) & $\mathrm{H}$ & IWP & \\
\hline Iniistius pavo (Valenciennes, 1840) & $\mathrm{H} / \mathrm{S}$ & $\mathrm{IP}$ & \\
\hline Labrichthys unilineatus (Guichenot, 1847) & $\mathrm{H}$ & IWP & \\
\hline Labroides bicolor Fowler \& Bean, 1928 & $\mathrm{H}$ & IWP & \\
\hline Labroides dimidiatus (Valenciennes, 1839) & $\mathrm{H}$ & IWP & $\bullet$ \\
\hline Labroides pectoralis Randall \& Springer, 1975 & $\mathrm{H}$ & IWP & \\
\hline Labropsis manabei Schmidt, 1930 & $\mathrm{H}$ & IWP & \\
\hline Labropsis xanthonota Randall, 1981 & $\mathrm{H}$ & IWP & \\
\hline Leptojulis cyanopleura (Bleeker, 1853) & $\mathrm{H}$ & IWP & \\
\hline Leptoscarus vaigiensis (Quoy \& Gaimard, 1824) & $\mathrm{H}$ & IWP & \\
\hline Macropharyngodon meleagris (Valenciennes, 1839) & $\mathrm{H}$ & IWP & \\
\hline Macropharyngodon negrosensis Herre, 1932 & $\mathrm{H}$ & IWP & $\bullet$ \\
\hline Macropharyngodon ornatus Randall, 1978 & $\mathrm{H}$ & IWP & \\
\hline Novaculichthys taeniourus (Lacépède, 1801) & $\mathrm{H} / \mathrm{S}$ & IWP & \\
\hline Oxycheilinus bimaculatus (Valenciennes, 1840) & $\mathrm{H}$ & IWP & \\
\hline Oxycheilinus celebicus (Bleeker, 1853) & $\mathrm{H}$ & IWP & \\
\hline Oxycheilinus digrammus (Lacépède, 1801) & $\mathrm{H}$ & IWP & \\
\hline Oxycheilinus unifasciatus (Streets, 1877) & $\mathrm{H}$ & IWP & \\
\hline Paracheilinus flavianalis Kuiter \& Allen, 1999 & $\mathrm{H}$ & IWP & \\
\hline Pseudocheilinus evanidus Jordan \& Evermann, 1903 & $\mathrm{H}$ & IWP & \\
\hline Pseudocheilinus hexataenia (Bleeker, 1857) & $\mathrm{H}$ & IWP & $\bullet$ \\
\hline Pseudocheilinus octotaenia Jenkins, 1901 & $\mathrm{H}$ & IWP & \\
\hline Pseudocoris yamashiroi (Schmidt, 1930) & $\mathrm{H}$ & IWP & \\
\hline Pseudodax moluccanus (Valenciennes, 1840) & $\mathrm{H}$ & IWP & \\
\hline Pteragogus cryptus Randall, 1981 & $\mathrm{H}$ & IWP & \\
\hline Pteragogus enneacanthus (Bleeker, 1851) & $\mathrm{H}$ & IWP & \\
\hline Pteragogus flagellifer (Valenciennes, 1839) & $\mathrm{H}$ & IWP & \\
\hline Scarus dimidiatus Bleeker, 1859 & $\mathrm{H}$ & IWP & \\
\hline Scarus flavipectoralis Schultz, 1958 & $\mathrm{H}$ & IWP & \\
\hline Scarus forsteni (Bleeker, 1861) & $\mathrm{H}$ & IWP & \\
\hline
\end{tabular}




\begin{tabular}{|c|c|c|c|}
\hline Species & Habitat code & $\begin{array}{l}\text { Biogeographic } \\
\text { code }\end{array}$ & $\begin{array}{l}\frac{0}{0} \\
\frac{0}{\infty} \\
\underline{D}\end{array}$ \\
\hline Scarus frenatus Lacépède, 1802 & $\mathrm{H}$ & IWP & \\
\hline Scarus ghobban Forsskål, 1775 & $\mathrm{H}$ & IP & - \\
\hline Scarus globiceps Valenciennes, 1840 & $\mathrm{H}$ & IWP & \\
\hline Scarus niger Forsskål, 1775 & $\mathrm{H}$ & IWP & \\
\hline Scarus oviceps Valenciennes, 1840 & $\mathrm{H}$ & IWP & \\
\hline Scarus prasiognathus Valenciennes, 1840 & $\mathrm{H}$ & IWP & \\
\hline Scarus psittacus Forsskål, 1775 & $\mathrm{H}$ & IWP & \\
\hline Scarus rivulatus Valenciennes, 1840 & $\mathrm{H}$ & IWP & - \\
\hline Scarus rubroviolaceus Bleeker, 1847 & $\mathrm{H}$ & IP & - \\
\hline Scarus schlegeli (Bleeker, 1861) & $\mathrm{H}$ & IWP & - \\
\hline Scarus spinus (Kner, 1868) & $\mathrm{H}$ & IWP & \\
\hline Scarus tricolor Bleeker, 1847 & $\mathrm{H}$ & IWP & \\
\hline Scarus xanthopleura Bleeker, 1853 & $\mathrm{H}$ & IWP & \\
\hline Stethojulis bandanensis (Bleeker, 1851) & $\mathrm{H}$ & IWP & \\
\hline Stethojulis interrupta (Bleeker, 1851) & $\mathrm{H}$ & IWP & - \\
\hline Stethojulis strigiventer (Bennett, 1832) & $\mathrm{H}$ & IWP & \\
\hline Stethojulis trilineata (Bloch \& Schneider, 1801) & $\mathrm{H}$ & IWP & \\
\hline Thalassoma amblycephalum (Bleeker, 1856) & $\mathrm{H}$ & IWP & - \\
\hline Thalassoma hardwicke (Bennett, 1829) & $\mathrm{H}$ & IWP & - \\
\hline Thalassoma jansenii (Bleeker, 1856) & $\mathrm{H}$ & IWP & \\
\hline Thalassoma lunare (Linnaeus, 1758) & $\mathrm{H}$ & IWP & - \\
\hline Thalassoma purpureum (Forsskål, 1775) & $\mathrm{H}$ & IWP & - \\
\hline Thalassoma quinquevittatum (Lay \& Bennett, 1839) & $\mathrm{H}$ & IWP & \\
\hline Thalassoma trilobatum (Lacépède, 1801) & $\mathrm{H}$ & IWP & \\
\hline Wetmorella albofasciata Schultz \& Marshall, 1954 & $\mathrm{H}$ & IWP & \\
\hline Wetmorella nigropinnata (Seale, 1901) & $\mathrm{H}$ & IWP & \\
\hline \multicolumn{4}{|l|}{ Family: Opistognathidae } \\
\hline Opistognathus darwiniensis Macleay, 1878 & $\mathrm{H} / \mathrm{S}$ & NA & - \\
\hline Opistognathus inornatus Ramsay \& Ogilby, 1887 & $\mathrm{~S}$ & WA & - \\
\hline Opistognathus reticeps Smith-Vaniz, 2004 & $\mathrm{~S}$ & NA & - \\
\hline Opistognathus reticulatus (McKay, 1969) & $\mathrm{S}$ & WA & - \\
\hline \multicolumn{4}{|l|}{ Family: Pinguipedidae } \\
\hline Parapercis clathrata Ogilby, 1910 & $\mathrm{H}$ & IWP & - \\
\hline Parapercis cylindrica (Bloch, 1797) & $\mathrm{H}$ & IWP & - \\
\hline Parapercis millepunctata $\left(\right.$ Günther, 1860) ${ }^{\mathrm{r}}$ & $\mathrm{H}$ & IWP & \\
\hline Parapercis multiplicata Randall, 1984 & $\mathrm{H}$ & IWP & \\
\hline Parapercis nebulosa (Quoy \& Gaimard, 1825) & $\mathrm{H} / \mathrm{S}$ & NA & - \\
\hline Parapercis pacifica Imamura \& Yoshino, 2007 & $\mathrm{H} / \mathrm{S}$ & IWP & \\
\hline Parapercis schauinslandii (Steindachner, 1900) & $\mathrm{H} / \mathrm{S}$ & IWP & \\
\hline Parapercis snyderi Jordan \& Starks, 1905 & $\mathrm{H} / \mathrm{S}$ & IWP & - \\
\hline \multicolumn{4}{|l|}{ Family: Trichonotidae } \\
\hline Trichonotus elegans Shimada \& Yoshino, 1984 & $\mathrm{H} / \mathrm{S}$ & IWP & \\
\hline Trichonotus setiger Bloch \& Schneider, 1801 & $\mathrm{H} / \mathrm{S}$ & IWP & $\bullet$ \\
\hline \multicolumn{4}{|l|}{ Family: Creediidae } \\
\hline Limnichthys nitidus Smith, 1958 & $\mathrm{H} / \mathrm{S}$ & IWP & \\
\hline \multicolumn{4}{|l|}{ Family: Uranoscopidae } \\
\hline Ichthyscopus fasciatus Haysom, 1957 & $\mathrm{~S}$ & IA & - \\
\hline Ichthyscopus insperatus Mees, 1960 & $\mathrm{~S}$ & NA & - \\
\hline Ichthyscopus spinosus Mees, 1960 & $\mathrm{~S}$ & WA & \\
\hline
\end{tabular}


Family: Blenniidae

Aspidontus dussumieri (Valenciennes, 1836)

Aspidontus taeniatus Quoy \& Gaimard, 1834

Atrosalarias fuscus (Rüppell, 1838) s

Blenniella chrysospilos (Bleeker, 1857)

Blenniella periophthalmus (Valenciennes, 1836)

Cirripectes alleni Williams, 1993

Cirripectes castaneus (Valenciennes, 1836)

Cirripectes filamentosus (Alleyne \& Macleay, 1877)

Cirripectes polyzona (Bleeker, 1868)

Cirripectes stigmaticus Strasburg \& Schultz, 1953

Ecsenius alleni Springer, 1988

Ecsenius bicolor (Day, 1888)

Ecsenius lineatus Klausewitz, 1962

Ecsenius lividanalis Chapman \& Schultz, 1952

Ecsenius namiyei (Jordan \& Evermann, 1902)

Ecsenius oculatus Springer, 1988

Ecsenius schroederi McKinney \& Springer, 1976

Ecsenius stictus Springer, 1988

Ecsenius trilineatus Springer, 1972

Ecsenius yaeyamaensis (Aoyagi, 1954)

Enchelyurus kraussii (Klunzinger, 1871)

Entomacrodus decussatus (Bleeker, 1858)

Exallias brevis (Kner, 1868)

Glyptoparus delicatulus Smith, 1959

Istiblennius edentulus (Forster \& Schneider, 1801)

Istiblennius lineatus (Valenciennes, 1836)

Istiblennius meleagris (Valenciennes, 1836)

Laiphognathus multimaculatus Smith, 1955

Meiacanthus atrodorsalis (Günther, 1877)

Meiacanthus ditrema Smith-Vaniz, 1976

Meiacanthus grammistes (Valenciennes, 1836)

Meiacanthus naevius Smith-Vaniz, 1987

Nannosalarias nativitatis (Regan, 1909)

Omobranchus ferox (Herre, 1927)

Omobranchus germaini (Sauvage, 1883)

Omobranchus lineolatus (Kner, 1868)

Omobranchus punctatus (Valenciennes, 1836)

Omobranchus rotundiceps (Macleay, 1881)

Omobranchus verticalis Springer \& Gomon, 1975

Petroscirtes breviceps (Valenciennes, 1836)

Petroscirtes mitratus Rüppell, 1830

Petroscirtes xestus Jordan \& Seale, 1906

Plagiotremus laudandus (Whitley, 1961)

Plagiotremus rhinorhynchos (Bleeker, 1852)

Plagiotremus tapeinosoma (Bleeker, 1857)

Salarias fasciatus (Bloch, 1786)

Salarias guttatus Valenciennes, 1836

Salarias patzneri Bath, 1992

Salarias sexfilum Günther, 1861

Salarias sinuosus Snyder, 1908
$\mathrm{P}$

$\mathrm{P}$

$\mathrm{H}$

$\mathrm{H}$

$\mathrm{H}$

$\mathrm{H}$

$\mathrm{H}$

$\mathrm{H}$

$\mathrm{H}$

$\mathrm{H}$

$\mathrm{H}$

$\mathrm{H}$

$\mathrm{H}$

$\mathrm{H}$

$\mathrm{H}$

$\mathrm{H}$

$\mathrm{H}$

$\mathrm{H}$

$\mathrm{H}$

$\mathrm{H}$

$\mathrm{H}$

$\mathrm{H}$

$\mathrm{H}$

$\mathrm{H}$

$\mathrm{H}$

$\mathrm{H}$

$\mathrm{H}$

$\mathrm{H}$

H

$\mathrm{H}$

$\mathrm{H}$

$\mathrm{H}$

$\mathrm{H}$

$\mathrm{E} / \mathrm{M}$

$\mathrm{H}$

E/M

$\mathrm{H} / \mathrm{S} / \mathrm{E}$

$\mathrm{H}$

S/E

$\mathrm{H}$

$\mathrm{H}$

$\mathrm{H}$

$\mathrm{H} / \mathrm{P}$

$\mathrm{H} / \mathrm{P}$

$\mathrm{H} / \mathrm{P}$

$\mathrm{H}$

$\mathrm{H}$

$\mathrm{H}$

$\mathrm{H}$

$\mathrm{H}$
IWP

IWP

IWP

IWP

IWP

WA

IWP

IWP

IWP

IWP

WA

IWP

IWP

IWP

IWP

IO

IWP

IWP

IWP

IWP

IWP

IWP

IWP

IWP

IWP

IWP

A

IWP

IWP

IWP

IWP

WA

IWP

IWP

IWP

IA

IWP

IWP

A

IWP

IWP

IWP

IWP

IWP

IWP

IWP

IWP

IA

IWP

IWP

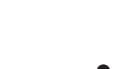

-

-

-

-

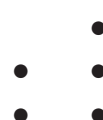

-

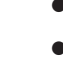

$\bullet$

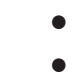

-

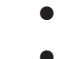

$\bullet$

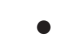

$\bullet$
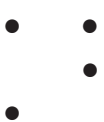

-

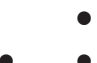

-

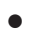

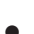
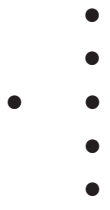

-

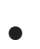

$\bullet$

$\bullet$

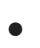

-
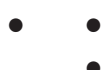

-

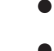




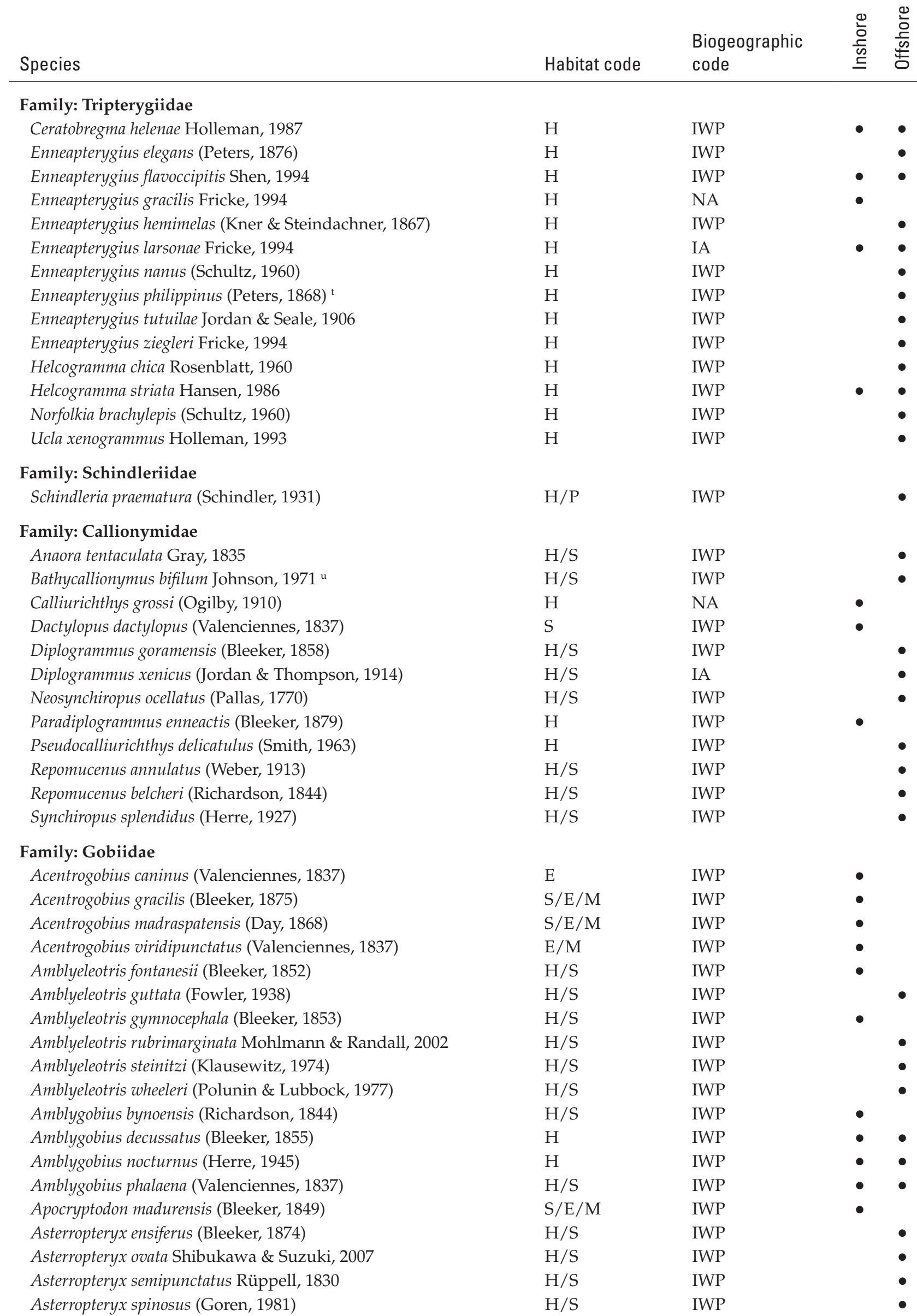




\begin{tabular}{|c|c|c|c|}
\hline Species & Habitat code & $\begin{array}{l}\text { Biogeographic } \\
\text { code }\end{array}$ & $\begin{array}{l}\text { 모 } \\
\text { 든 } \\
\stackrel{\text { S }}{ }\end{array}$ \\
\hline Bathygobius coalitus (Bennett, 1832) & $\mathrm{H}$ & IWP & \\
\hline Bathygobius cocosensis (Bleeker, 1854) & $\mathrm{H}$ & IWP & $\bullet$ \\
\hline Bathygobius cyclopterus (Valenciennes, 1837) & $\mathrm{H}$ & IWP & \\
\hline Bathygobius fuscus (Rüppell, 1830) & $\mathrm{H}$ & IWP & $\bullet$ \\
\hline Bathygobius laddi (Fowler, 1931) & $\mathrm{H}$ & IWP & $\bullet$ \\
\hline Boleophthalmus caeruleomaculatus McCulloch \& Waite, 1918 & S & A & $\bullet$ \\
\hline Bryaninops amplus Larson, 1985 & $\mathrm{H} / \mathrm{EZ}$ & IWP & $\bullet$ \\
\hline Bryaninops erythrops (Jordan \& Seale, 1906) & $\mathrm{H} / \mathrm{EZ}$ & IWP & \\
\hline Bryaninops isis Larson, 1985 & $\mathrm{H} / \mathrm{EZ}$ & IWP & \\
\hline Bryaninops loki Larson, 1985 & $\mathrm{H} / \mathrm{EZ}$ & IWP & $\bullet$ \\
\hline Bryaninops natans Larson, 1985 & $\mathrm{H} / \mathrm{EZ}$ & IWP & \\
\hline Bryaninops nexus Larson, 1987 & $\mathrm{H} / \mathrm{EZ}$ & IWP & \\
\hline Bryaninops ridens Smith, 1959 & $\mathrm{H} / \mathrm{EZ}$ & IWP & \\
\hline Bryaninops yongei (Davis \& Cohen, 1968) & $\mathrm{H} / \mathrm{EZ}$ & IWP & \\
\hline Cabillus lacertops Smith, 1959 & $\mathrm{H}$ & IWP & \\
\hline Cabillus tongarevae (Fowler, 1927) & $\mathrm{H}$ & IWP & \\
\hline Callogobius maculipinnis (Fowler, 1918) & $\mathrm{H}$ & IWP & \\
\hline Callogobius sclateri (Steindachner, 1880) & $\mathrm{H}$ & IWP & $\bullet$ \\
\hline Cryptocentroides insignis (Seale, 1910) & $\mathrm{E}$ & IWP & $\bullet$ \\
\hline Cryptocentrus caeruleomaculatus (Herre, 1933) & $\mathrm{H} / \mathrm{S}$ & IWP & $\bullet$ \\
\hline Cryptocentrus cinctus (Herre, 1936) & $\mathrm{H} / \mathrm{S}$ & IWP & $\bullet$ \\
\hline Cryptocentrus fasciatus (Playfair, 1866) & $\mathrm{H} / \mathrm{S}$ & IWP & $\bullet$ \\
\hline Cryptocentrus insignitus (Whitley, 1956) & $\mathrm{S}$ & NA & $\bullet$ \\
\hline Cryptocentrus leptocephalus (Bleeker, 1876) & $\mathrm{S} / \mathrm{E} / \mathrm{M}$ & IWP & $\bullet$ \\
\hline Cryptocentrus leucostictus (Günther, 1872) & $\mathrm{H} / \mathrm{S}$ & IWP & \\
\hline Cryptocentrus strigilliceps (Jordan \& Seale, 1906) & $\mathrm{H} / \mathrm{S}$ & IWP & $\bullet$ \\
\hline Cryptocentrus tentaculatus Hoese \& Larson, 2004 & $\mathrm{H} / \mathrm{S}$ & NA & $\bullet$ \\
\hline Ctenogobiops aurocingulus (Herre, 1935) & $\mathrm{H} / \mathrm{S}$ & IWP & \\
\hline Ctenogobiops feroculus Lubbock \& Polunin, 1977 & $\mathrm{H} / \mathrm{S}$ & IWP & \\
\hline Ctenogobiops maculosus (Fourmanoir, 1955) & $\mathrm{H} / \mathrm{S}$ & IWP & \\
\hline Ctenogobiops pomastictus Lubbock \& Polunin, 1977 & $\mathrm{H} / \mathrm{S}$ & IWP & $\bullet$ \\
\hline Ctenogobiops tangaroai Lubbock \& Polunin, 1977 & $\mathrm{H} / \mathrm{S}$ & IWP & \\
\hline Discordipinna griessingeri Hoese \& Fourmanoir, 1978 & $\mathrm{H}$ & IWP & \\
\hline Drombus halei Whitley, 1935 & $\mathrm{H}$ & NA & $\bullet$ \\
\hline Drombus triangularis (Weber, 1909) & $\mathrm{E} / \mathrm{M}$ & IWP & $\bullet$ \\
\hline Echinogobius hayashii Iwata, Hosoya \& Niimura, 1998 & $\mathrm{H}$ & IWP & \\
\hline Eugnathogobius polylepis (Wu \& Ni, 1985) & $\mathrm{E}$ & IWP & $\bullet$ \\
\hline Eviota afelei Jordan \& Seale, 1906 & $\mathrm{H}$ & IWP & \\
\hline Eviota albolineata Jewett \& Lachner, 1983 & $\mathrm{H}$ & IWP & \\
\hline Eviota cometa Jewett \& Lachner, 1983 & $\mathrm{H}$ & IWP & \\
\hline Eviota distigma Jordan \& Seale, 1906 & $\mathrm{H}$ & IWP & \\
\hline Eviota herrei Jordan \& Seale, 1906 & $\mathrm{H}$ & IWP & \\
\hline Eviota cf. indica Lachner \& Karnella, 1980 & $\mathrm{H}$ & $\mathrm{IO}$ & \\
\hline Eviota infulata (Smith, 1957) & $\mathrm{H}$ & IWP & \\
\hline Eviota inutilis Whitley, 1943 & $\mathrm{H}$ & WA & $\bullet$ \\
\hline Eviota lachdeberei Giltay, 1933 & $\mathrm{H}$ & IWP & \\
\hline Eviota latifasciata Jewett \& Lachner, 1983 & $\mathrm{H}$ & IWP & \\
\hline Eviota melasma Lachner \& Karnella, 1980 & $\mathrm{H}$ & IWP & \\
\hline Eviota nebulosa Smith, 1958 & $\mathrm{H}$ & IWP & \\
\hline Eviota nigriventris Giltay, 1933 & $\mathrm{H}$ & IWP & \\
\hline Eviota pellucida Larson, 1976 & $\mathrm{H}$ & IWP & \\
\hline Eviota prasina (Klunzinger, 1871) & $\mathrm{H}$ & IWP & \\
\hline
\end{tabular}




\begin{tabular}{|c|c|c|c|}
\hline Species & Habitat code & $\begin{array}{l}\text { Biogeographic } \\
\text { code }\end{array}$ & 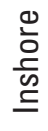 \\
\hline Eviota prasites Jordan \& Seale, 1906 & $\mathrm{H}$ & IWP & \\
\hline Eviota punctulata Jewett \& Lachner, 1983 & $\mathrm{H}$ & IWP & \\
\hline Eviota queenslandica Whitley, 1932 & $\mathrm{H}$ & IWP & • \\
\hline Eviota sebreei Jordan \& Seale, 1906 & $\mathrm{H}$ & IWP & $\bullet$ \\
\hline Eviota sigillata Jewett \& Lachner, 1983 & $\mathrm{H}$ & IWP & \\
\hline Eviota smaragdus Jordan \& Seale, 1906 & $\mathrm{H}$ & IWP & \\
\hline Eviota sparsa Jewett \& Lachner, 1983 & $\mathrm{H}$ & IWP & \\
\hline Eviota spilota Lachner \& Karnella, 1980 & $\mathrm{H}$ & IWP & \\
\hline Eviota storthynx (Rofen, 1959) & $\mathrm{H}$ & IWP & • \\
\hline Eviota zebrina (Lachner \& Karnella, 1978) & $\mathrm{H}$ & IWP & $\bullet$ \\
\hline Eviota zonura Jordan \& Seale, 1906 & $\mathrm{H}$ & IWP & $\bullet$ \\
\hline Exyrias belissimus (Smith, 1959) & $\mathrm{H} / \mathrm{S}$ & IWP & $\bullet$ \\
\hline Favonigobius melanobranchus (Fowler, 1934) & $\mathrm{S} / \mathrm{E}$ & IWP & $\bullet$ \\
\hline Feia nympha Smith, 1959 & $\mathrm{H} / \mathrm{S}$ & IWP & \\
\hline Fusigobius duospilus Hoese \& Reader, 1985 & $\mathrm{H} / \mathrm{S}$ & IWP & \\
\hline Fusigobius humeralis (Randall, 2001) & $\mathrm{H} / \mathrm{S}$ & IWP & \\
\hline Fusigobius inframaculatus (Randall, 1994) & $\mathrm{H} / \mathrm{S}$ & IWP & $\bullet$ \\
\hline Fusigobius longispinus Goren, 1978 & $\mathrm{H} / \mathrm{S}$ & IWP & \\
\hline Fusigobius neophytus (Günther, 1877) & $\mathrm{H} / \mathrm{S}$ & IWP & \\
\hline Fusigobius signipinnis Hoese \& Obika, 1988 & $\mathrm{H} / \mathrm{S}$ & IWP & \\
\hline Glossogobius circumspectus (Macleay, 1883) & $\mathrm{S} / \mathrm{E} / \mathrm{M}$ & IWP & • \\
\hline Glossogobius giuris (Hamilton, 1822) & $\mathrm{S} / \mathrm{E} / \mathrm{FW}$ & IWP & $\bullet$ \\
\hline Gnatholepis anjerensis (Bleeker, 1851) & $\mathrm{H} / \mathrm{S}$ & IWP & \\
\hline Gnatholepis argus Larson \& Buckle, 2005 & $\mathrm{H} / \mathrm{S}$ & NA & • \\
\hline Gnatholepis cauerensis (Bleeker, 1853) & $\mathrm{H} / \mathrm{S}$ & IWP & \\
\hline Gobiodon citrinus (Rüppell, 1838) & $\mathrm{H} / \mathrm{EZ}$ & IWP & \\
\hline Gobiodon histrio (Valenciennes, 1837) & $\mathrm{H} / \mathrm{EZ}$ & IWP & \\
\hline Gobiodon micropus Günther, 1861 & $\mathrm{H} / \mathrm{EZ}$ & IWP & \\
\hline Gobiodon okinawae Sawada, Arai \& Abe, 1972 & $\mathrm{H} / \mathrm{EZ}$ & IWP & \\
\hline Gobiodon quinquestrigatus (Valenciennes, 1837) & $\mathrm{H} / \mathrm{EZ}$ & IWP & $\bullet$ \\
\hline Gobiodon rivulatus (Rüppell, 1830) & $\mathrm{H} / \mathrm{EZ}$ & IWP & \\
\hline Gobiodon spilophthalmus Fowler, 1944 & $\mathrm{H} / \mathrm{EZ}$ & IWP & \\
\hline Gobiopsis angustifrons Lachner \& McKinney, 1978 & $\mathrm{H}$ & IWP & \\
\hline Gobiopsis aporia Lachner \& McKinney, 1978 & $\mathrm{H}$ & IWP & $\bullet$ \\
\hline Gobiopsis arenaria (Snyder, 1908) & $\mathrm{H}$ & IA & $\bullet$ \\
\hline Grallenia arenicola Shibukawa \& Iwata, 2007 & S & IP & $\bullet$ \\
\hline Istigobius decoratus (Herre, 1927) & $\mathrm{H} / \mathrm{S}$ & IWP & - \\
\hline Istigobius diadema (Steindachner, 1876) & $S$ & IWP & $\bullet$ \\
\hline Istigobius goldmanni (Bleeker, 1852) & $\mathrm{H} / \mathrm{S}$ & IWP & $\bullet$ \\
\hline Istigobius nigroocellatus (Günther, 1873) & $\mathrm{H} / \mathrm{S}$ & IWP & $\bullet$ \\
\hline Istigobius ornatus (Rüppell, 1830) & $\mathrm{S} / \mathrm{E} / \mathrm{M}$ & IWP & $\bullet$ \\
\hline Istigobius rigilius (Herre, 1953) & $\mathrm{H} / \mathrm{S}$ & IWP & \\
\hline Istigobius spence (Smith, 1947) & $\mathrm{H} / \mathrm{S}$ & IWP & $\bullet$ \\
\hline Koumansetta rainfordi (Whitley, 1940) & $\mathrm{H}$ & IWP & \\
\hline Lotilia graciliosa Klausewitz, 1960 & $\mathrm{H} / \mathrm{S}$ & IWP & \\
\hline Luposicya lupus Smith, 1959 & $\mathrm{H} / \mathrm{S} / \mathrm{EZ}$ & IWP & \\
\hline Macrodontogobius wilburi Herre, 1936 & $\mathrm{H} / \mathrm{S}$ & IWP & $\bullet$ \\
\hline Minysicya caudimaculata Larson, 2002 & $\mathrm{H}$ & IWP & \\
\hline Mugilogobius filifer Larson, 2001 & $\mathrm{E} / \mathrm{M} / \mathrm{FW}$ & IA & $\bullet$ \\
\hline Mugilogobius littoralis Larson, 2001 & $\mathrm{H} / \mathrm{E} / \mathrm{M}$ & NA & • \\
\hline Myersina macrostoma Herre, 1934 & S & IWP & $\bullet$ \\
\hline Myersina nigrivirgata Akihito \& Meguro, 1983 & $\mathrm{H} / \mathrm{S}$ & IWP & $\bullet$ \\
\hline
\end{tabular}




\begin{tabular}{|c|c|c|c|}
\hline Species & Habitat code & $\begin{array}{l}\text { Biogeographic } \\
\text { code }\end{array}$ & 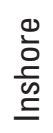 \\
\hline Oplopomops diacanthus (Schultz, 1943) & $\mathrm{H} / \mathrm{S}$ & IWP & \\
\hline Oplopomus caninoides (Bleeker, 1852) & $S$ & IWP & $\bullet$ \\
\hline Pandaka lidwilli (McCulloch, 1917) & $\mathrm{P} / \mathrm{E} / \mathrm{M}$ & IWP & - \\
\hline Paragobiodon echinocephalus (Rüppell, 1830) & H/EZ & IWP & - \\
\hline Paragobiodon lacunicola (Kendall \& Goldsborough, 1911) & H/EZ & IWP & \\
\hline Paragobiodon melanosoma (Bleeker, 1852) & H/EZ & IWP & \\
\hline Paragobiodon modestus (Regan, 1908) & H/EZ & IWP & \\
\hline Paragobiodon xanthosoma (Bleeker, 1852) & H/EZ & IWP & - \\
\hline Periophthalmodon freycineti (Valenciennes, 1824) & $\mathrm{S} / \mathrm{E} / \mathrm{A}$ & IWP & - \\
\hline Periophthalmus argentilineatus Valenciennes, 1837 & $\mathrm{~S} / \mathrm{E} / \mathrm{A}$ & IWP & $\bullet$ \\
\hline Periophthalmus darwini Larson \& Takita, 2004 & $\mathrm{E} / \mathrm{M} / \mathrm{A}$ & NA & $\bullet$ \\
\hline Periophthalmus minutus Eggert, 1935 & $\mathrm{~S} / \mathrm{E} / \mathrm{A}$ & IWP & $\bullet$ \\
\hline Periophthalmus novaeguineaensis Eggert, $1935^{\mathrm{v}}$ & $\mathrm{S} / \mathrm{E} / \mathrm{A}$ & IA & $\bullet$ \\
\hline Phyllogobius platycephalops (Smith, 1964) & $\mathrm{H} / \mathrm{EZ}$ & IWP & \\
\hline Pleurosicya coerulea Larson, 1990 & $\mathrm{H} / \mathrm{EZ}$ & IWP & $\bullet$ \\
\hline Pleurosicya elongata Larson, 1990 & $\mathrm{H} / \mathrm{EZ}$ & IWP & $\bullet$ \\
\hline Pleurosicya fringilla Larson, 1990 & $\mathrm{H} / \mathrm{EZ}$ & IWP & - \\
\hline Pleurosicya labiata (Weber, 1913) & $\mathrm{H} / \mathrm{EZ}$ & IWP & \\
\hline Pleurosicya micheli Fourmanoir, 1971 & $\mathrm{H} / \mathrm{EZ}$ & IWP & \\
\hline Pleurosicya mossambica Smith, 1959 & H/EZ & IWP & \\
\hline Pleurosicya muscarum (Jordan \& Seale, 1906) & H/EZ & IWP & \\
\hline Pleurosicya plicata Larson, 1990 & H/EZ & IWP & \\
\hline Pleurosicya prognatha Goren, 1984 & H/EZ & IO & \\
\hline Priolepis cincta (Regan, 1908) & $\mathrm{H}$ & IWP & \\
\hline Priolepis compita Winterbottom, 1985 & $\mathrm{H}$ & IWP & $\bullet$ \\
\hline Priolepis nuchifasciata (Günther, 1873) & $\mathrm{H}$ & IWP & $\bullet$ \\
\hline Priolepis semidoliata (Valenciennes, 1837) & $\mathrm{H}$ & IWP & $\bullet$ \\
\hline Pseudogobius poicilosoma (Bleeker, 1849) & S/E & IWP & $\bullet$ \\
\hline Scartelaos histophorus (Valenciennes, 1837) & $\mathrm{S} / \mathrm{E} / \mathrm{M}$ & IWP & $\bullet$ \\
\hline Signigobius biocellatus Hoese \& Allen, 1977 & $\mathrm{H} / \mathrm{S}$ & IWP & \\
\hline Stonogobiops larsonae (Allen, 1999) & $\mathrm{H} / \mathrm{S}$ & WA & - \\
\hline Sueviota atrinasa Winterbottom \& Hoese, 1988 & $\mathrm{H}$ & WA & - \\
\hline Sueviota lachneri Winterbottom \& Hoese, 1988 & $\mathrm{H}$ & IWP & \\
\hline Taenioides mordax (De Vis, 1883) & S/E & NA & - \\
\hline Trimma agrena Winterbottom \& Chen, 2004 & $\mathrm{H}$ & IWP & - \\
\hline Trimma caesiura Jordan \& Seale, 1906 & $\mathrm{H} / \mathrm{P}$ & IWP & \\
\hline Trimma emeryi Winterbottom, 1985 & $\mathrm{H} / \mathrm{P}$ & IWP & \\
\hline Trimma flavatrum Hagiwara \& Winterbottom, 2007 & $\mathrm{H}$ & IWP & \\
\hline Trimma lantana Winterbottom \& Villa, 2003 & $\mathrm{H}$ & IWP & \\
\hline Trimma macrophthalmum (Tomiyama, 1936) & $\mathrm{H}$ & IWP & \\
\hline Trimma milta Winterbottom, 2002 & $\mathrm{H}$ & IWP & \\
\hline Trimma cf. naudei Smith, 1957 & $\mathrm{H}$ & $\mathrm{U}$ & \\
\hline Trimma okinawae (Aoyagi, 1949) & $\mathrm{H}$ & IWP & • \\
\hline Trimma cf. sheppardi Winterbottom, 1984 & $\mathrm{H}$ & $\mathrm{U}$ & \\
\hline Trimma stobbsi Winterbottom, 2001 & $\mathrm{H}$ & IWP & \\
\hline Trimma striatum (Herre, 1945) & $\mathrm{H} / \mathrm{P}$ & IWP & \\
\hline Trimma taylori Lobel, 1979 & $\mathrm{H} / \mathrm{P}$ & IWP & \\
\hline Trimma tevegae Cohen \& Davis, 1969 & $\mathrm{H} / \mathrm{P}$ & IWP & \\
\hline Trimmatom eviotops (Schultz, 1943) & $\mathrm{H}$ & IWP & \\
\hline Trimmatom macropodus Winterbottom, 1989 & $\mathrm{H}$ & IWP & \\
\hline Trimmatom nanus Winterbottom \& Emery, 1981 & $\mathrm{H}$ & IWP & \\
\hline Trimmatom zapotes Winterbottom, 1989 & $\mathrm{H}$ & IWP & \\
\hline
\end{tabular}




\begin{tabular}{|c|c|c|c|}
\hline Species & Habitat code & $\begin{array}{l}\text { Biogeographic } \\
\text { code }\end{array}$ & 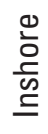 \\
\hline Valenciennea alleni Hoese \& Larson, 1994 & $\mathrm{H} / \mathrm{S} / \mathrm{P}$ & NA & $\bullet$ \\
\hline Valenciennea longipinnis (Lay \& Bennett, 1839) & $\mathrm{H} / \mathrm{S} / \mathrm{P}$ & IWP & • \\
\hline Valenciennea muralis (Valenciennes, 1837) & $\mathrm{H} / \mathrm{S} / \mathrm{P}$ & IWP & - \\
\hline Valenciennea parva Hoese \& Larson, 1994 & $\mathrm{H} / \mathrm{S} / \mathrm{P}$ & IWP & \\
\hline Valenciennea puellaris (Tomiyama, 1956) & $\mathrm{H} / \mathrm{S} / \mathrm{P}$ & IWP & \\
\hline Valenciennea sexguttata (Valenciennes, 1837) & $\mathrm{H} / \mathrm{S} / \mathrm{P}$ & IWP & \\
\hline Valenciennea strigata (Broussonet, 1782) & $\mathrm{H} / \mathrm{S} / \mathrm{P}$ & IWP & \\
\hline Vanderhorstia ambanoro (Fourmanoir, 1957) & $\mathrm{H} / \mathrm{S}$ & IWP & \\
\hline Vanderhorstia ornatissima Smith, 1959 & $\mathrm{H} / \mathrm{S}$ & IWP & \\
\hline Yongeichthys nebulosus (Forsskål, 1775) & $\mathrm{S} / \mathrm{E} / \mathrm{M}$ & IWP & $\bullet$ \\
\hline \multicolumn{4}{|l|}{ Family: Eleotridae } \\
\hline Butis butis (Hamilton, 1822) & $\mathrm{S} / \mathrm{E} / \mathrm{M}$ & IWP & - \\
\hline Calumia godeffroyi (Günther, 1877) & $\mathrm{H}$ & IWP & \\
\hline Calumia profunda Larson \& Hoese, 1980 & $\mathrm{H}$ & IWP & \\
\hline Hypseleotris compressa (Krefft, 1864) & $\mathrm{P} / \mathrm{E} / \mathrm{FW}$ & IA & $\bullet$ \\
\hline Ophiocara porocephala (Valenciennes, 1837) & $\mathrm{E} / \mathrm{FW}$ & IWP & $\bullet$ \\
\hline Prionobutis microps (Weber, 1907) & $\mathrm{S} / \mathrm{E} / \mathrm{M}$ & NA & $\bullet$ \\
\hline \multicolumn{4}{|l|}{ Family: Xenisthmidae } \\
\hline Xenisthmus chi Gill \& Hoese, 2004 & $\mathrm{H}$ & WA & \\
\hline Xenisthmus clarus (Jordan \& Seale, 1906) & $\mathrm{H} / \mathrm{S}$ & IWP & \\
\hline Xenisthmus polyzonatus (Klunzinger, 1871) & $\mathrm{H} / \mathrm{S}$ & IWP & \\
\hline Xenisthmus semicinctus Gill \& Hoese, 2004 & $\mathrm{H}$ & WA & \\
\hline \multicolumn{4}{|l|}{ Family: Kraemeriidae } \\
\hline Kraemeria merensis Whitley, 1935 & $\mathrm{~S} / \mathrm{E}$ & NA & \\
\hline \multicolumn{4}{|l|}{ Family: Microdesmidae } \\
\hline Aioliops novaeguineaensis Rennis \& Hoese, 1987 & $\mathrm{H} / \mathrm{P}$ & IA & \\
\hline Aioliops tetrophthalmus Rennis \& Hoese, 1987 & $\mathrm{H} / \mathrm{P}$ & NA & \\
\hline Gunnellichthys copleyi (Smith, 1951) & $\mathrm{H} / \mathrm{P}$ & IWP & \\
\hline Gunnellichthys monostigma Smith, 1958 & $\mathrm{H} / \mathrm{P}$ & IWP & \\
\hline Gunnellichthys pleurotaenia Bleeker, 1858 & $\mathrm{H} / \mathrm{P}$ & IWP & \\
\hline Nemateleotris magnifica Fowler, 1938 & $\mathrm{H} / \mathrm{P}$ & IWP & \\
\hline Parioglossus nudus Rennis \& Hoese, 1985 & $\mathrm{H} / \mathrm{E} / \mathrm{P}$ & IWP & $\bullet$ \\
\hline Parioglossus palustris (Herre, 1945) & $\mathrm{P} / \mathrm{E}$ & IWP & $\bullet$ \\
\hline Parioglossus philippinus (Herre, 1945) & $\mathrm{H} / \mathrm{P} / \mathrm{E}$ & IWP & $\bullet$ \\
\hline Ptereleotris evides (Jordan \& Hubbs, 1925) & $\mathrm{H} / \mathrm{P}$ & IWP & \\
\hline Ptereleotris hanae (Jordan \& Snyder, 1901) & $\mathrm{H} / \mathrm{S} / \mathrm{P}$ & IWP & $\bullet$ \\
\hline Ptereleotris heteroptera (Bleeker, 1855) & $\mathrm{H} / \mathrm{P}$ & IWP & \\
\hline Ptereleotris microlepis (Bleeker, 1856) & $\mathrm{H} / \mathrm{P}$ & IWP & $\bullet$ \\
\hline Ptereleotris zebra (Fowler, 1938) & $\mathrm{H} / \mathrm{P}$ & IWP & \\
\hline \multicolumn{4}{|l|}{ Family: Kurtidae } \\
\hline Kurtus gulliveri Castelnau, 1878 & $\mathrm{E} / \mathrm{FW}$ & IA & $\bullet$ \\
\hline \multicolumn{4}{|l|}{ Family: Acanthuridae } \\
\hline Acanthurus bariene Lesson, 1831 & $\mathrm{H}$ & IWP & \\
\hline Acanthurus blochii Valenciennes, 1835 & $\mathrm{H}$ & IWP & \\
\hline Acanthurus dussumieri Valenciennes, 1835 & $\mathrm{H}$ & IWP & $\bullet$ \\
\hline Acanthurus grammoptilus Richardson, 1843 & $\mathrm{H}$ & IWP & $\bullet$ \\
\hline Acanthurus lineatus (Linnaeus, 1758) & $\mathrm{H}$ & IWP & \\
\hline Acanthurus mata Cuvier, 1829 & $\mathrm{H}$ & IWP & \\
\hline Acanthurus nigricans (Linnaeus, 1758) & $\mathrm{H}$ & IWP & $\bullet$ \\
\hline
\end{tabular}




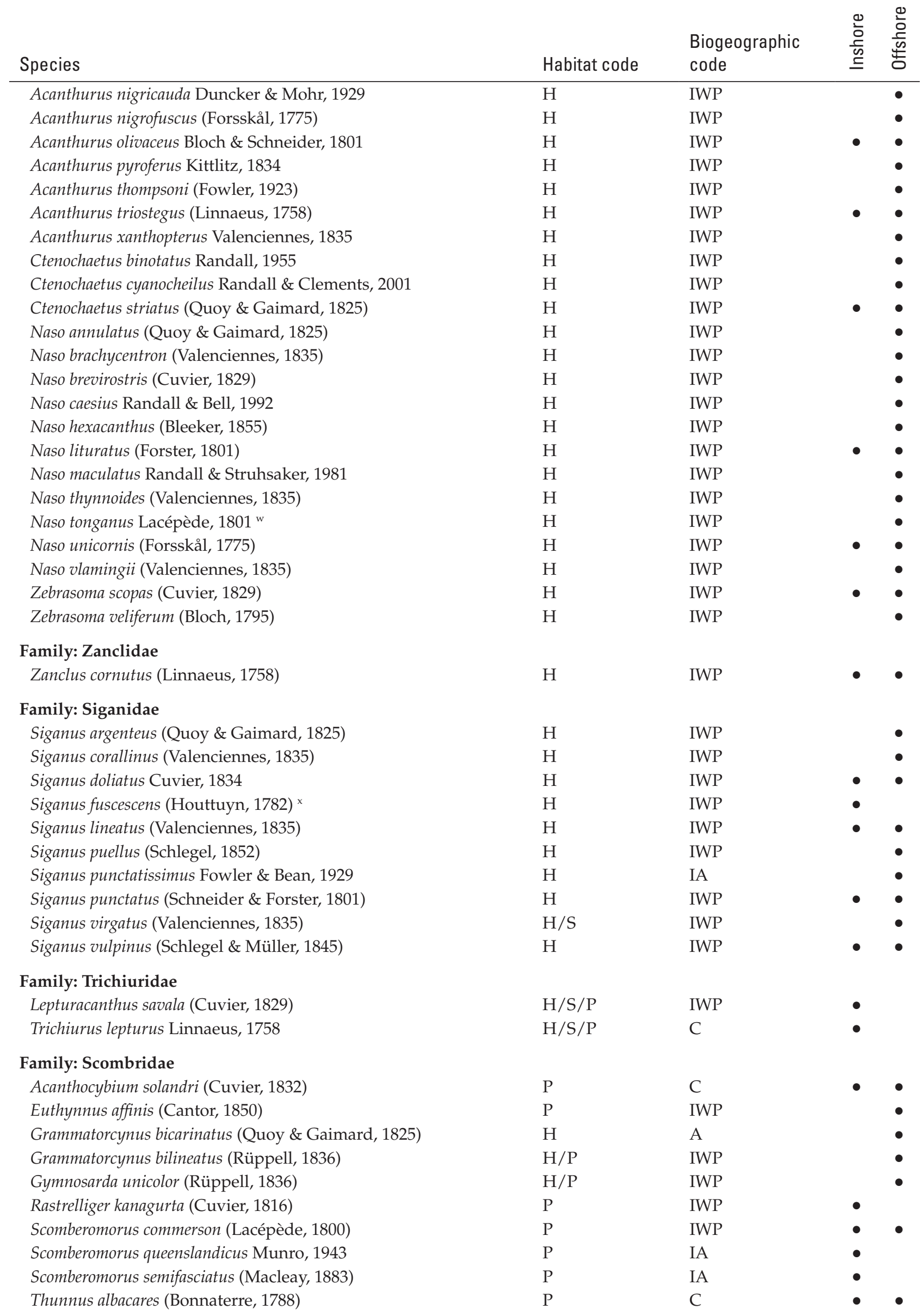




\begin{tabular}{|c|c|c|c|}
\hline Species & Habitat code & $\begin{array}{l}\text { Biogeographic } \\
\text { code }\end{array}$ & 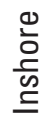 \\
\hline \multicolumn{4}{|l|}{ Family: Istiophoridae } \\
\hline Istiophorus platypterus (Shaw \& Nodder, 1792) & $\mathrm{P}$ & $\mathrm{C}$ & $\bullet$ \\
\hline Makaira nigricans Lacépède, $1802^{\text {y }}$ & $\mathrm{P}$ & $\mathrm{C}$ & \\
\hline \multicolumn{4}{|l|}{ Family: Nomeidae } \\
\hline Psenes arafurensis Günther, 1889 & $\mathrm{P}$ & $\mathrm{C}$ & \\
\hline Psenes cyanophrys Valenciennes, 1833 & $\mathrm{P}$ & C & \\
\hline \multicolumn{4}{|l|}{ Family: Citharidae } \\
\hline Brachypleura novaezeelandiae Günther, 1862 & $\mathrm{~S}$ & IWP & $\bullet$ \\
\hline \multicolumn{4}{|l|}{ Family: Bothidae } \\
\hline Asterorhombus cocosensis (Bleeker, 1855) & $\mathrm{H} / \mathrm{S}$ & IWP & \\
\hline Bothus mancus (Broussonet, 1782) & $\mathrm{H} / \mathrm{S}$ & IWP & \\
\hline Bothus pantherinus (Rüppell, 1830) & $\mathrm{H} / \mathrm{S}$ & IWP & \\
\hline \multicolumn{4}{|l|}{ Family: Paralichthyidae } \\
\hline Pseudorhombus arsius (Hamilton, 1822) & $\mathrm{S} / \mathrm{E}$ & IWP & $\bullet$ \\
\hline Pseudorhombus jenynsii (Bleeker, 1855) & $\mathrm{S} / \mathrm{E}$ & A & $\bullet$ \\
\hline \multicolumn{4}{|l|}{ Family: Pleuronectidae } \\
\hline Psammodiscus ocellatus Günther, 1862 & $\mathrm{~S}$ & IA & $\bullet$ \\
\hline \multicolumn{4}{|l|}{ Family: Samaridae } \\
\hline Samariscus triocellatus Woods, 1966 & $\mathrm{H} / \mathrm{S}$ & IWP & \\
\hline \multicolumn{4}{|l|}{ Family: Soleidae } \\
\hline Aseraggodes melanostictus (Peters, 1877) ${ }^{\mathrm{z}}$ & $\mathrm{S} / \mathrm{E}$ & IA & $\bullet$ \\
\hline Aseraggodes whitleyi (Chabanaud, 1950) & $\mathrm{S}$ & NA & \\
\hline Brachirus aspilos (Bleeker, 1852) & $S$ & IWP & \\
\hline Dagetichthys marginata (Boulenger, 1900) & $\mathrm{S}$ & IWP & \\
\hline Dexillus muelleri (Steindachner, 1879) & $\mathrm{S} / \mathrm{E}$ & IWP & $\bullet$ \\
\hline Pardachirus pavoninus (Lacépède, 1802) & $\mathrm{H} / \mathrm{S}$ & IWP & $\bullet$ \\
\hline Pardachirus rautheri (Chabanaud, 1931) & S/E & IA & $\bullet$ \\
\hline Phyllichthys punctatus McCulloch, 1916 & $S$ & WA & $\bullet$ \\
\hline Rendahlia jaubertensis (Rendahl, 1921) & $\mathrm{S}$ & IA & $\bullet$ \\
\hline Soleichthys heterorhinos (Bleeker, 1856) & $\mathrm{H} / \mathrm{S}$ & IWP & $\bullet$ \\
\hline Zebrias munroi (Whitley, 1966) & $S$ & NA & $\bullet$ \\
\hline \multicolumn{4}{|l|}{ Family: Cynoglosidae } \\
\hline Cynoglossus bilineatus (Lacépède, 1802) & $\mathrm{S}$ & IWP & $\bullet$ \\
\hline Cynoglossus maccullochi Norman, 1926 & $\mathrm{~S}$ & A & $\bullet$ \\
\hline Cynoglossus maculipinnis Rendahl, 1921 & S & A & $\bullet$ \\
\hline Paraplagusia bilineata (Bloch, 1787) & $\mathrm{S}$ & IWP & $\bullet$ \\
\hline Paraplagusia longirostris Chapleau, Renaud \& Kailola, 1991 & S & IA & $\bullet$ \\
\hline Paraplagusia sinerama Chaplau \& Renaud, 1993 & S & IA & $\bullet$ \\
\hline \multicolumn{4}{|l|}{ Family: Triacanthidae } \\
\hline Triacanthus biaculeatus (Bloch, 1786) & S & IWP & $\bullet$ \\
\hline Triacanthus nieuhofi Bleeker, 1852 & $\mathrm{~S}$ & IWP & $\bullet$ \\
\hline Tripodichthys blochii (Bleeker, 1852) & S & IWP & $\bullet$ \\
\hline \multicolumn{4}{|l|}{ Family: Balistidae } \\
\hline Abalistes stellatus (Anonymous, 1798) & $\mathrm{H} / \mathrm{S}$ & IWP & $\bullet$ \\
\hline Balistapus undulatus (Park, 1797) & $\mathrm{H}$ & IWP & $\bullet$ \\
\hline Balistoides conspicillum (Bloch \& Schneider, 1801) & $\mathrm{H}$ & IWP & \\
\hline Balistoides viridescens (Bloch \& Schneider, 1801) & $\mathrm{H}$ & IWP & • \\
\hline
\end{tabular}




\begin{tabular}{|c|c|c|c|}
\hline Species & Habitat code & $\begin{array}{l}\text { Biogeographic } \\
\text { code }\end{array}$ & 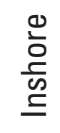 \\
\hline Melichthys niger (Bloch, 1786) & $\mathrm{H}$ & $\mathrm{C}$ & \\
\hline Melichthys vidua (Richardson, 1845) & $\mathrm{H}$ & IWP & \\
\hline Odonus niger (Rüppell, 1837) & $\mathrm{H}$ & IWP & \\
\hline Pseudobalistes flavimarginatus (Rüppell, 1829) & $\mathrm{H}$ & IWP & \\
\hline Pseudobalistes fuscus (Bloch \& Schneider, 1801) & $\mathrm{H}$ & IWP & \\
\hline Rhinecanthus aculeatus (Linnaeus, 1758) & $\mathrm{H}$ & IWP & \\
\hline Rhinecanthus rectangulus (Bloch \& Schneider, 1801) & $\mathrm{H}$ & IWP & \\
\hline Rhinecanthus verrucosus (Linnaeus, 1758) & $\mathrm{H}$ & IWP & \\
\hline Sufflamen bursa (Bloch \& Schneider, 1801) & $\mathrm{H}$ & IWP & \\
\hline Sufflamen chrysopterum (Bloch \& Schneider, 1801) & $\mathrm{H}$ & IWP & \\
\hline Sufflamen fraenatum (Latreille, 1804) & $\mathrm{H}$ & IWP & \\
\hline \multicolumn{4}{|l|}{ Family: Monacanthidae } \\
\hline Acreichthys radiatus (Popta, 1901) & $\mathrm{H}$ & IWP & \\
\hline Aluterus scriptus (Osbeck, 1765) & $\mathrm{H}$ & $\mathrm{C}$ & \\
\hline Amanses scopas (Cuvier, 1829) & $\mathrm{H} / \mathrm{S}$ & IWP & \\
\hline Cantherhines dumerilii (Hollard, 1854) & $\mathrm{H}$ & IP & \\
\hline Cantherhines pardalis (Rüppell, 1837) & $\mathrm{H}$ & IWP & \\
\hline Chaetodermis penicilligera (Cuvier, 1816) & S & IWP & $\bullet$ \\
\hline Colurodontis paxmani Hutchins, 1977 & SG & NA & $\bullet$ \\
\hline Monacanthus chinensis (Osbeck, 1765) & $\mathrm{H} / \mathrm{S} / \mathrm{SG} / \mathrm{E}$ & IWP & $\bullet$ \\
\hline Oxymonacanthus longirostris (Bloch \& Schneider, 1801) & $\mathrm{H}$ & IWP & - \\
\hline Paraluteres prionurus (Bleeker, 1851) & $\mathrm{H}$ & IWP & \\
\hline Paramonacanthus choirocephalus (Bleeker, 1852) & $\mathrm{S}$ & IA & - \\
\hline Paramonacanthus filicauda (Günther, 1880) & $\mathrm{S}$ & IA & - \\
\hline Paramonacanthus pusillus (Rüppell, 1829) & $\mathrm{S}$ & IWP & - \\
\hline Pervagor janthinosoma (Bleeker, 1854) & $\mathrm{H}$ & IWP & \\
\hline Pervagor melanocephalus (Bleeker, 1853) & $\mathrm{H}$ & IWP & \\
\hline Pervagor nigrolineatus (Herre, 1927) & $\mathrm{H}$ & IWP & \\
\hline Pseudalutarius nasicornis (Temminck \& Schlegel, 1850) & S/E/SG & IWP & \\
\hline \multicolumn{4}{|l|}{ Family: Ostraciidae } \\
\hline Lactoria cornuta (Linnaeus, 1758) & $\mathrm{H} / \mathrm{S}$ & IWP & • \\
\hline Lactoria diaphana (Bloch \& Schneider, 1801) & $\mathrm{H} / \mathrm{S} / \mathrm{E}$ & IWP & $\bullet$ \\
\hline Ostracion cubicus Linnaeus, 1758 & $\mathrm{H}$ & IWP & $\bullet$ \\
\hline Ostracion meleagris Shaw, 1796 & $\mathrm{H}$ & IWP & \\
\hline Ostracion solorensis Bleeker, 1853 & $\mathrm{H}$ & IWP & \\
\hline Rhynchostracion rhinorhynchos (Bleeker, 1852) & $\mathrm{H} / \mathrm{S}$ & IWP & - \\
\hline Rhynchostracion nasus (Bloch, 1785) & $\mathrm{H}$ & IWP & $\bullet$ \\
\hline \multicolumn{4}{|l|}{ Family: Tetraodontidae } \\
\hline Arothron hispidus (Linnaeus, 1758) & $\mathrm{H} / \mathrm{E}$ & IWP & - \\
\hline Arothron immaculatus Bloch \& Schneider, 1801) & $\mathrm{S}$ & IWP & \\
\hline Arothron manilensis (Procé, 1822) & $\mathrm{S} / \mathrm{E}$ & IWP & • \\
\hline Arothron тарра (Lesson, 1831) & $\mathrm{H}$ & IWP & \\
\hline Arothron meleagris (Anonymous, 1798) & $\mathrm{H}$ & IP & \\
\hline Arothron nigropunctatus (Bloch \& Schneider, 1801) & $\mathrm{H}$ & IWP & $\bullet$ \\
\hline Arothron reticularis (Bloch \& Schneider, 1801) & $\mathrm{S} / \mathrm{E} / \mathrm{M}$ & IWP & $\bullet$ \\
\hline Arothron stellatus (Bloch \& Schneider, 1801) & $\mathrm{H}$ & IWP & $\bullet$ \\
\hline Canthigaster bennetti (Bleeker, 1854) & $\mathrm{H} / \mathrm{S}$ & IWP & \\
\hline Canthigaster janthinoptera (Bleeker, 1855) & $\mathrm{H}$ & IWP & \\
\hline Canthigaster papua (Bleeker, 1848) & $\mathrm{H}$ & IWP & \\
\hline Canthigaster valentini (Bleeker, 1853) & $\mathrm{H}$ & IWP & \\
\hline
\end{tabular}




\begin{tabular}{|c|c|c|c|}
\hline Species & Habitat code & $\begin{array}{l}\text { Biogeographic } \\
\text { code }\end{array}$ & 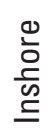 \\
\hline Chelonodon patoca (Hamilton, 1822) & $\mathrm{S} / \mathrm{E}$ & IWP & $\bullet$ \\
\hline Feroxodon multistriatus (Richardson, 1854) & $\mathrm{S}$ & NA & $\bullet$ \\
\hline Lagocephalus lunaris (Bloch \& Schneider, 1801) & $\mathrm{S} / \mathrm{P}$ & IWP & $\bullet$ \\
\hline Marilyna darwinii (Castelnau, 1873) & $\mathrm{H} / \mathrm{S}$ & IA & $\bullet$ \\
\hline Marilyna meraukensis (De Beaufort, 1955) & $\mathrm{S} / \mathrm{E} / \mathrm{M}$ & IA & $\bullet$ \\
\hline \multicolumn{4}{|l|}{ Family: Diodontidae } \\
\hline Cyclichthys orbicularis (Bloch, 1785) & $\mathrm{S} / \mathrm{P}$ & IWP & $\bullet$ \\
\hline Diodon hystrix Linnaeus, 1758 & $\mathrm{H}$ & $\mathrm{C}$ & $\bullet$ \\
\hline Diodon liturosus Shaw, 1804 & $\mathrm{H}$ & IWP & $\bullet$ \\
\hline Tragulichthys jaculiferus (Cuvier, 1818) & S & $\mathrm{A}$ & $\bullet$ \\
\hline
\end{tabular}

Historically reported as Carcharinus sealei (see White 2012).

Historically reported as Albula forsteri (see ABRS 2014).

Historically reported as Liocranium praepositus (Motomura et al. 2008).

Probably only Paracentropogon vespa in Australia (J. Johnson personal communication) but both species retained here based on ABRS (2014).

Includes records attributed to Pelates octolineatus (see ABRS 2014).

Historically reported as Apogon nigripinnis (see Fraser and Allen 2010).

Historically reported as Foa brachygrammus (see Fraser and Randall 2011).

May be junior synonym of Gymnapogon vanderbilti or a cryptic species complex (see Mabuchi et al. 2014).

Possibly misidentified Pseudamia gelatinosa.

Historically reported as Siphamia versicolor (see Gon and Allen 2012).

Includes records attributed to Taeniamia biguttata (see Fraser 2013).

Historically reported as Sillago maculata (see ABRS 2014).

Often misidentified and reported as Leiognathus decorus.

Includes records attributed to Secutor interruptus (see ABRS 2014).

Sometimes misidentified and reported as Kyphosus gibsoni (see ABRS 2014).

Some doubt over this old record.

Historically reported as Cetoscarus bicolor.

Possible misidentification.

Historically reported as Atrosalarias holomelas (see ABRS 2014).

historically reported as Enneapterygius minutus (see ABRS 2014).

Sometimes misidentified and reported as Bathycallionymus moretonensis.

Likely includes Periophthalmus murdyi and Periophthalmus takita (see Jaafar and Larson 2008).

Possibly includes misidentified Naso tuberosus.

Includes records attributed to Siganus canaliculatus (see ABRS 2014).

Historically reported as Maikara mazara (see Collette et al. 2006).

Possibly part of Aseraggodes 'klunzingeri' species complex (= Leptachirus spp.; see Randall 2007). 
APPENDIX 2 Species of fishes excluded from the Kimberley Project Area dataset based on dubious identifications.

Species

Comment

\section{INSHORE RECORDS}

Carcharhinus brachyurus (Günther, 1870)

Gymnothorax woodwardi McCulloch, 1912

Ambassis agassizii Steindachner, 1867

Vincentia punctata (Klunzinger, 1880)

Chelmon rostratus (Linnaeus, 1758)

Amphiprion percula (Lacépède, 1802)

Parablennius postoculomaculatus Bath \& Hutchins, 1986

Eviota bimaculata Lachner \& Karnella, 1980

Psammogobius biocellatus (Valenciennes, 1837)

Giuris margaritacea (Valenciennes, 1837)

Marilyna pleurosticta (Günther, 1872)

\section{OFFSHORE RECORDS}

Moringua bicolor Kaup, 1856

Gymnothorax moluccensis (Bleeker, 1864)

Gymnothorax richardsonii (Bleeker, 1852)

Uropterygius macrocephalus (Bleeker, 1864)

Malvoliophis pinguis (Günther, 1872)

Antennarius randalli Allen, 1970

Pseudoplesiops annae (Weber, 1913)

Amblypomacentrus breviceps (Schlegel \& Müller, 1839)

Amphiprion percula (Lacépède, 1802)

Chrysiptera flavipinnis (Allen \& Robertson, 1974)

Neopomacentrus bankieri (Richardson, 1846)

Neopomacentrus violascens (Bleeker, 1848)

Pomacentrus auriventris Allen, 1991

Pomacentrus brachialis Cuvier, 1830

Halichoeres argus (Bloch \& Schneider, 1801)

Halichoeres miniatus (Valenciennes, 1839)

Labropsis australis Randall, 1981

Novaculoides macrolepidotus (Bloch, 1791)

Oxycheilinus orientalis (Günther, 1862)

Hipposcarus harid (Forsskål, 1775)

Scarus altipinnis (Steindachner, 1879)

Rhabdoblennius ellipes cf.

Amblyeleotris fasciata (Herre, 1953)

Arcygobius baliurus (Valenciennes, 1837)

Eviota bifasciata Lachner \& Karnella, 1980

Fusigobius gracilis (Randall, 2001)

Silhouettea evanida Larson \& Miller, 1986

Gunnellichthys irideus Smith, 1958

Zebrasoma rostratum (Günther, 1875)

Canthidermis maculatus (Bloch, 1786)

Cantherhines fronticinctus (Günther, 1866)
Well outside known range, probably incorrect ID. Well outside known range, probably incorrect ID. Well outside known range, probably incorrect ID. Well outside known range, probably incorrect ID. Probably C. marginalis

Probably A. ocellaris

Well outside known range, probably incorrect ID. Well outside known range, probably incorrect ID. Well outside known range, probably incorrect ID. Well outside known range, probably incorrect ID. Well outside known range, probably incorrect ID.

Well outside known range, probably incorrect ID. Well outside known range, probably incorrect ID. Well outside known range, probably incorrect ID. Well outside known range, probably incorrect ID. Well outside known range, probably incorrect ID. Well outside known range, probably incorrect ID. Well outside known range, probably incorrect ID. Well outside known range, probably incorrect ID. Probably A. ocellaris

Well outside known range, probably incorrect ID. Well outside known range, probably incorrect ID. Well outside known range, probably incorrect ID. Well outside known range, probably incorrect ID. Well outside known range, probably incorrect ID. Well outside known range, probably incorrect ID. Probably H. margaritaceus or H. nebulosus Well outside known range, probably incorrect ID. Well outside known range, probably incorrect ID. Well outside known range, probably incorrect ID. Well outside known range, probably incorrect ID. Well outside known range, probably incorrect ID. Well outside known range, probably incorrect ID. Well outside known range, probably incorrect ID. Well outside known range, probably incorrect ID. Well outside known range, probably incorrect ID. Well outside known range, probably incorrect ID. Well outside known range, probably incorrect ID. Well outside known range, probably incorrect ID. Well outside known range, probably incorrect ID. Well outside known range, probably incorrect ID. Well outside known range, probably incorrect ID. 